\title{
Computational Image Quality
}

\author{
T.J.W.M. Janssen
}


The work described in this thesis has been carried out at IPO, Center for User-System Interaction,

Eindhoven, the Netherlands.

(C) T.J.W.M. Janssen, 1999.

\section{CIP-DATA LIBRARY TECHNISCHE UNIVERSITEIT EINDHOVEN}

Janssen, Timotheus J.W.M.

Computational image quality /

by Timotheus Johannes Wilhelmus Maria Janssen. -

Eindhoven: Technische Universiteit Eindhoven, 1999. -

ISBN 90-386-0563-3

NUGI 832

Keywords: Image quality / Colour reproduction 


\title{
Computational Image Quality
}

\author{
PROEFSCHRIFT
}

ter verkrijging van de graad van doctor aan de Technische Universiteit Eindhoven, op gezag van de Rector Magnificus, prof.dr. M. Rem, voor een commissie aangewezen door het College voor

Promoties in het openbaar te verdedigen op dinsdag 2 november 1999 om 16.00 uur

door

Timotheus Johannes Wilhelmus Maria Janssen

geboren te Laren 
Dit proefschrift is goedgekeurd door de promotoren:

prof.dr. A. Kohlrausch

en

prof. R.J. Watt

Copromotor:

dr.ir. F.J.J. Blommaert

\section{Cover:}

Five versions of a digitised image of Bryce Canyon, obtained by scaling CIE chroma, $C_{u v}^{*}$ of the colours of the pixels constituting the image. The scale factor used is 0.5 (upper left), 0.7 (upper right), 1.0 (centre), 1.4 (lower left), and 2.0 (lower right). This type of manipulation was also used in the experiments described in chapter 2. 


\section{Acknowledgments}

I very much enjoyed the time I spent at IPO doing the research described in this thesis. For this I would like to thank the following people.

First and foremost, Frans Blommaert. I can't think of any better Ph.D. supervisor. Armin Kohlrausch, for his involvement which far exceeded that what is to be expected of a first promotor. Roger Watt, for his useful comments on the thesis and for his hospitality during my visit to Stirling. Ina Klein Teeselink, a roommate too special to describe. Sergej Yendrikhovskij and Huib de Ridder, for the discussions we had and for their useful comments. Martin Boschman, Truus Gruisen and Roelof Hamberg, for their technical support. And finally, Leon Luwijs, Wijnand IJsselsteijn, Lydia Meesters, Najoua Belaïd, Toon van Dijk, and Ton Roelofs. I want to thank them for their contribution to the excellent atmosphere at IPO. 


\section{Contents}

1 Introduction 1

2 Image quality semantics $\quad 7$

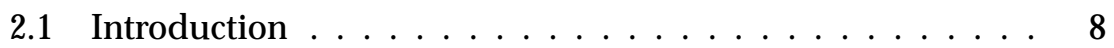

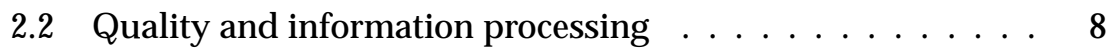

2.2.1 Understanding information-processing systems . . 8

2.2.2 The quality of information . . . . . . . . . . . 9

2.2 .3 The quality of images . . . . . . . . . . . 10

2.3 Image quality semantics $\ldots \ldots \ldots \ldots$

2.3.1 Image processing by the visuo-cognitive system . . . 10

2.3.2 Naturalness, usefulness and quality . . . . . . . 12

2.4 Experiments . . . . . . . . . . . . . . . . . . . . 13

2.4.1 Experiment 1: Influences of naturalness and usefulness on image quality . . . . . . . . . . . . . 13

2.4.2 Experiment 2: Image quality regarded as a compromise between naturalness and usefulness . . . . . . . 18

2.5 Concluding remarks . . . . . . . . . . . . . . 24

3 Visual metrics: discriminative power through flexibility 27

3.1 Introduction . . . . . . . . . . . . . . . . . 27

3.2 The usefulness of flexibility . . . . . . . . . . . . . . 29

3.3 Recipe for an optimal metric . . . . . . . . . . . . . . . 33 
3.3.1 Problem specification $\ldots \ldots \ldots 33$

3.3 .2 Solution . . . . . . . . . . . . . . . 36

3.3.3 Flexibility versus rigidity: performances compared . 39

3.3.4 Concluding remarks . . . . . . . . . . . . 40

3.4 Vision \& visual memory . . . . . . . . . . . . . . . . . 42

3.4.1 Visual identification: vision versus memory . . . . 42

3.4.2 Calibrating visual metrics . . . . . . . . . . . . . 44

3.4.3 Uncalibrated visual metrics: partial flexibility . . . . 45

3.5 Conclusions . . . . . . . . . . . . . . . . . . 46

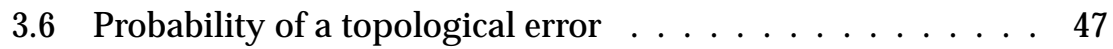

4 Predicting the usefulness and naturalness of colour reproductions 49

4.1 Introduction . . . . . . . . . . . . . 50

4.2 Metrics for brightness and colour . . . . . . . . . . 53

4.3 Predicting usefulness $\ldots \ldots \ldots \ldots 6$

4.3 .1 Discriminability . . . . . . . . . . . . . . . 58

4.3.2 Results and discussion . . . . . . . . . . . . . . 61

4.4 Predicting naturalness . . . . . . . . . . . . . . . 65

4.4.1 The construction of memory standards . . . . . . 66

4.4.2 Matching perceived object colours with memory standards . . . . . . . . . . . . . . . 69

4.4.3 Results and discussion . . . . . . . . . . . 70

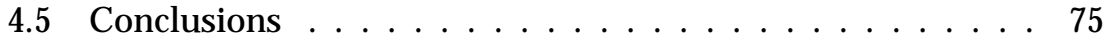

5 Image quality revisited 77

5.1 Introduction . . . . . . . . . . . . . . . 77

5.2 What is image quality? . . . . . . . . . . . . 78

5.3 The internal quantification of attributes $\ldots \ldots \ldots$ 
5.4 An optimal metric for overall discriminability $\ldots \ldots$. . . . 82

5.4.1 A measure for overall discriminability . . . . . . . . 82

5.4.2 Optimising overall discriminability . . . . . . . 84

5.5 An optimal metric for overall identifiability . . . . . . . . 85

5.5.1 The accumulation of scale value distributions . . . 85

5.5.2 A decision rule for identification . . . . . . . . 87

5.5.3 A measure for overall identifiability . . . . . . . . 88

5.5.4 Optimising overall identifiability . . . . . . . . . . 89

5.6 Estimating the number of discriminable and identifiable items 90

5.7 Partial flexibility . . . . . . . . . . . . . . . . 91

5.7.1 Discriminability for partial flexibility . . . . . . . 93

5.7.2 Identifiability for partial flexibility . . . . . . . . . 94

5.7.3 Re-estimating the number of discriminable and identifiable items . . . . . . . . . . . . . 94

5.7.4 Optimising the degree of flexibility . . . . . . . . . 97

5.8 Application: black-and-white images of natural scenes . . . 99

5.9 Conclusions . . . . . . . . . . . . . . . . . . . . . . . . 108

6 Epilogue 109

$\begin{array}{ll}\text { Bibliography } & 113\end{array}$

$\begin{array}{ll}\text { Sample stimuli } & 117\end{array}$

$\begin{array}{ll}\text { Summary } & 125\end{array}$

$\begin{array}{ll}\text { Samenvatting } & 127\end{array}$

$\begin{array}{ll}\text { Biography } & 129\end{array}$ 


\section{Chapter 1}

\section{Introduction}

Over recent decades the role of images in the communication of information has steadily grown. Advances in technologies underlying the capture, transfer, storage, and display of images have created a situation in which the use of images as a means of communicating information has become technologically and economically feasible. More importantly, however, images are in many situations an extremely efficient means to communicate information, as may be witnessed by the proverb "a picture is worth a thousand words". Without a doubt, this has been the most prominent factor pushing the above technological development.

Notwithstanding the above technological advances, the current state-ofthe-art still requires that certain compromises be made in the design of algorithms and devices for capture, transfer, storage, and display of images. Examples of such compromises are temporal resolution versus noise sensitivity (for capture), spatial resolution versus image size (for transfer and storage), and luminance range versus gamut (for displays). These and similar choices will, when made, affect the appearance of reproduced images, as well as the impression of how well the images are reproduced. To make optimal choices, it is therefore necessary to have knowledge of how particular design choices translate into the appearance of images and subsequently into the impression of how well these images are reproduced. In a nutshell, this is the central question of all image quality research.

Current image quality research can be divided into two fundamentally different approaches. The first approach focusses on experimental evaluation (Roufs 1993). A typical setup would include a small group of human subjects judging quality, and possibly some related attributes such as sharpness, contrast, or colourfulness, of a set of displayed images which are ma- 
nipulated to simulate the effects of several different design choices. In this way, the influence of these design choices on image quality can be measured and, by means of interpolation, approximately optimal choices can be made. Two serious drawbacks of this approach are: (1) it is extremely time-consuming, as well as tiresome for the participating subjects; and (2) the obtained knowledge cannot easily be generalised since all relations found between design choice and image quality are descriptive rather than based on an understanding. As a result, in a single series of experiments only a small fraction of the possible design space can be investigated, and in practice the experimental procedure must be repeated for almost every possible set of design choices.

The second approach tries to address these drawbacks by means of the development of models which describe the influences of several physical image characteristics on image quality, usually through a set of image attributes thought to determine image quality. When the influence of a set of design choices on physical image characteristics is known, these models can be used to predict image quality instead of having to measure image quality experimentally. Two different types of models can be distinguished here. Both types share the common characteristic that image quality is expressed in terms of the visibility of distortions, or artefacts, introduced in the image as a result of certain design choices. Examples of such distortions are flickering, blockiness, noisiness, or colour shifts. A hypothetical version of the image devoid of any such visible distortions is regarded as the "original", that is, the version of the image assumed to be of highest quality, and the visibility of distortions in the image, referred to as impairment, is used as a measure of quality degradation. The fundamental difference between the two types of model lies in how this impairment is calculated.

In the first type of model, physiologically or psychophysically inspired models of early visual processing are used to calculate impairment from a difference between two versions of an image, for example the "input" or "unprocessed" version, which is substituted for the original, and the "output" or "processed" version of a certain device or image processing algorithm. A well-known example of this approach is the JND-map (justnoticeable differences map) presented by Daly (1993). The two most important drawbacks of this approach are: (1) it is unclear what exactly the "unprocessed" version of an image is; and (2) what in fact is calculated here is the visible difference between two versions of an image, not image quality itself, and it remains unclear how the translation from visible difference to image quality should be performed. Usually this translation 
is made by means of fitting certain model parameters to experimentally obtained quality judgments of human subjects.

The second type of model differs from the first type in the sense that it tries to estimate visibility of distortions directly from the image instead of using a visible difference with an original. In this type of model, visible distortions of an image, such as unsharpness or noisiness, are predicted by estimating relevant physical attributes of the image, such as blur and noise spread. Using psychophysically established relations, these estimated physical attributes are then translated to visibility of distortions in the image. Finally, image quality is expressed in terms of these visible distortions using some kind of combination rule. The work presented by Nijenhuis \& Blommaert (1997) is a good example of this approach. The uncertain translation from visibility of distortions to image quality, which usually must be fitted to experimentally obtained data, remains an important drawback of this approach.

As may be concluded from the above description of the state-of-theart in image quality modelling, images are primarily regarded as twodimensional signals. Similarly, early visual processing is regarded as signal processing, with image quality being determined by a set of characteristics of the resulting output signal. There are a few serious shortcomings to this view, the most important of which is the fact that the fundamental question "What is image quality?" is never asked, nor answered. To give an answer to this question, based on a thorough understanding of visuocognitive processing and the role of images therein, is the very aim of this thesis.

The approach we will pursue in this thesis distinguishes itself from the above approaches in four fundamental ways:

- We will regard images not as signals but instead as carriers of visual information. Since an image is the result of the optical imaging process, which maps physical scene properties onto a two-dimensional luminance distribution, it encodes important and useful information about the geometry of the scene and the properties of the objects located within this scene.

- We will regard visuo-cognitive processing not as signal processing but instead as information processing. Following Marr (1982) and Newell (1990), we will regard vision and cognition as the processes in which, first, physical object properties, or correlates of these, are 
reconstructed from a two-dimensional luminance distribution using a set of dedicated computational algorithms and, second, resulting descriptions are interpreted by comparing them with descriptions stored in memory.

- We will regard visuo-cognitive processing not as an isolated process but instead as an essential stage in human interaction with the environment. Descriptions of scene content, as produced by visuocognitive processing, are used as input to subsequent stages in the process of interaction with the environment, such as semantic processing and action. Hence, visuo-cognitive processing plays a vital role within the interaction process.

- We will regard the quality of an image not in terms of the visibility of distortions in this image but instead in terms of the adequacy of this image as input to the vision stage of the interaction process. The basic definition of quality we will use is formulated in terms of the degree to which imposed requirements are satisfied. When images are considered to be input to the interaction process, requirements must be imposed on these images to guarantee the successfulness of the interaction process. In this view, the degree to which an image satisfies these requirements determines the quality of this image.

The structure of this thesis is as follows. In chapter 2 Image quality semantics we formulate an answer to the question "What is image quality?" based on the philosophy outlined in the above four points. In this chapter, we give a description of image quality in terms of two components, usefulness, that is, the precision of the internal representation of the image, and naturalness, that is, the degree of match between the internal representation of the image and representations stored in memory. The results of two series of experiments are used to demonstrate the validity of this concept.

Chapter 3 Visual metrics: discriminative power through flexibility focusses on the internal quantification of outside world attributes. Using a rather technical view on visual processing, we regard vision primarily as a process in which attributes of items in the outside world are measured and internally quantified with the aim to discriminate and/or identify these items. In chapter 3 we show that the scale function of metrics optimised with respect to these tasks should be (partially) flexible. Furthermore, we show that such metrics exhibit properties resembling phenomena such as adaptation, crispening, and constancy. 
A straightforward implementation of the image quality concept of chapter 2 using the partially flexible metrics of chapter 3 is the topic of chapter 4 Predicting the usefulness and naturalness of colour reproductions. In chapter 4 a measure for usefulness is developed, based on the overall discriminability of the items in the image. Furthermore, a measure for naturalness of the grass, skin, and sky areas of the image is developed, based on memory standards for grass, skin, and sky colour. These memory standards are themselves constructed from the grass, skin, and sky areas of a large set of images.

As its title suggests, chapter 5 Image quality revisited returns to the concept for image quality introduced in chapter 2. Following a strict, top-down analysis the entire trajectory is completed from the semantics of image quality down to the development of algorithms for the prediction of usefulness, naturalness, and image quality. Chapter 5 therefore contains a complete, thorough and explicit description of image quality according to the four-point philosophy outlined above. 


\title{
Chapter 2
}

\section{Image quality semantics}

\begin{abstract}
In this paper we will discuss image quality in the context of the visuocognitive system as an information-processing system. To this end, we subdivide the information-processing as performed by the visuo-cognitive system into three distinct processes: (1) the construction of a internal representation of the image; (2) the interpretation of this representation by means of a confrontation with memory; and (3) task-directed semantic processing of the interpreted scene in order to formulate a proper response.

A successful completion of these processes can only be ensured when two main requirements are satisfied: (1) the internal representation of the image should be sufficiently precise; and (2) the degree of correspondence between the internal representation and "knowledge of reality" as stored in memory should be high.

We then relate these requirements to the attributes "usefulness" and "naturalness" of the image, and give a functional description of image quality in terms of naturalness and usefulness. To conclude, experimental results supporting this description of image quality will be discussed.
\end{abstract}

${ }^{0}$ This chapter is a slightly modified version of Janssen \& Blommaert (1997). 


\subsection{Introduction}

A major part of research activity in the field of image quality is directed towards the development of reliable, widely applicable, instrumental image quality measures. There are two important reasons for developing instrumental measures: (1) quality evaluation by means of subjective assessment tests is quite expensive and time consuming; and (2) a posteriori assessing the image quality of a given design does not allow for an a priori optimisation of this design, thus condemning the design of image (re-)production systems to remain an iterative procedure.

At present, much of the research concerning instrumental image quality measures is based upon an approach which can be characterised as a "signal evaluation" approach. In this approach, the image is regarded as a complex signal which deviates more or less from the complex signal that represents the ideal or "original" image. Images are defined in the physical or perceptual domain, in the latter case using models of the earliest stages of visual perception, and quality measures are defined as distances in an appropriate function space, for example, Euclidean distance between actual and original image.

In contrast to this approach, we regard the processing of images by the visuo-cognitive system not as the evaluation of complex signals but instead as the processing of visual information. Realising that this information processing is an essential part of an observer's interaction with his environment we characterise the quality of an image in a more meaningful manner as the degree to which the image can be successfully exploited by the observer. We will therefore consider the visuo-cognitive system (1) as an information processing system (Marr 1982, Barrow \& Tenenbaum 1986, Eimer 1990, Watt 1991); and (2) as an integral part of an observer's interaction with his environment (Bruce \& Green 1985, Gibson 1950, Gibson 1966, Gibson 1979).

\subsection{Quality and information processing}

\subsubsection{Understanding information-processing systems}

We adopt the general viewpoint in computational cognition (Newell \& Simon 1972, Newell 1990) that (1) the visuo-cognitive system can be con- 
sidered to be an information-processing system; and (2) informationprocessing systems can only be completely understood when they are understood at three distinct levels. These levels are (1) the semantic level, that is, the level describing the system in terms of computational goals and strategies; (2) the algorithmic level, that is, the level describing the implementation of the computational theory into algorithms and associated representations; and (3) the level of physical implementation, that is, the level describing the physical implementation of these algorithms and representations ${ }^{1}$.

As stated above, any information-processing system can only be completely understood when it can be appropriately described at all three levels, which for reasons of simplicity may be designated as the levels of "what \& why", "how" and "where". The approach we choose to pursue here is strictly top-down, that is, we first try to gain a fundamental understanding of the "what \& why" of the processing of images by the visuo-cognitive system in order to arrive at an understanding of what image quality is, and then proceed with the "how" and "where". Our present purposes are therefore served best with a description of visuo-cognitive information-processing at the semantic level.

\subsubsection{The quality of information}

At a semantic level, the interaction of an observer with his environment can be described by a cycle consisting of three activities: (1) perception, that is, acquiring information from the environment and constructing an internal representation from it; (2) cognition, that is, interpreting the obtained internal representation; and (3) action, that is, responding appropriately to this interpretation ${ }^{2}$.

At this point, we may already infer that in order to ensure a proper response to occurrences in the outside world, certain requirements must be imposed upon the information which is acquired from the environment. When the quality of information is considered to be the degree to which these requirements are satisfied, we arrive at two important conclusions:

\footnotetext{
${ }^{1}$ Marr (1982) refers to these levels as the level of the computational theory, the level of representation and algorithm, and the level of the hardware implementation, respectively. The semantic level therefore corresponds to Marr's computational level.

${ }^{2}$ Throughout this thesis we will use the term cognition rather loosely to refer to almost the entire set of processes following perception and preceding action. Note, however, that action may depend on other inputs as well, such as emotions.
} 
(1) the quality of information can only be defined within the context of an observer interacting with his environment; and (2) the quality of information refers to the appropriateness of this information as a basis for a proper response to outside world occurrences.

\subsubsection{The quality of images}

Realising that images are the medium for visual information, we now focus our attention on the question what requirements should be imposed upon an image. At first thought, the requirements that a "good" image should satisfy would seem to be precision and reliability. The above outlined ideas, however, lead to a somewhat more complicated requirement: for an image to be of "good" quality, the observer's interpretation of this image should be successful, that is, should with high probability be correct. The imposition of this requirement is justified by realising that it is of vital importance that there is no discrepancy between "what really is there" and what the observer assumes is there. The next section will focus on the question how this can be optimally secured.

\subsection{Image quality semantics}

\subsubsection{Image processing by the visuo-cognitive system}

We will subdivide the processing of images by the visuo-cognitive system in three distinct processes (Marr 1982, Barrow \& Tenenbaum 1986): (1) perception, that is, the construction of a internal representation of the image using primarily low-level knowledge of the visual world; (2) interpretation, that is, the confrontation ("matching") of this internal representation with memory representations; and (3) task-directed semantic processing of the interpreted scene in order to formulate a response. A diagrammatical depiction of the processing of images by the visuo-cognitive system is shown in figure 2.1.

The collection of memory representations of the outside world, which we will from this point onwards refer to as "knowledge of reality", we regard as well-defined but nevertheless fuzzy. As an example of this, consider that although observers "know" what the prototypical characteristics of a certain object are, they usually are unable to define a clear distinction 


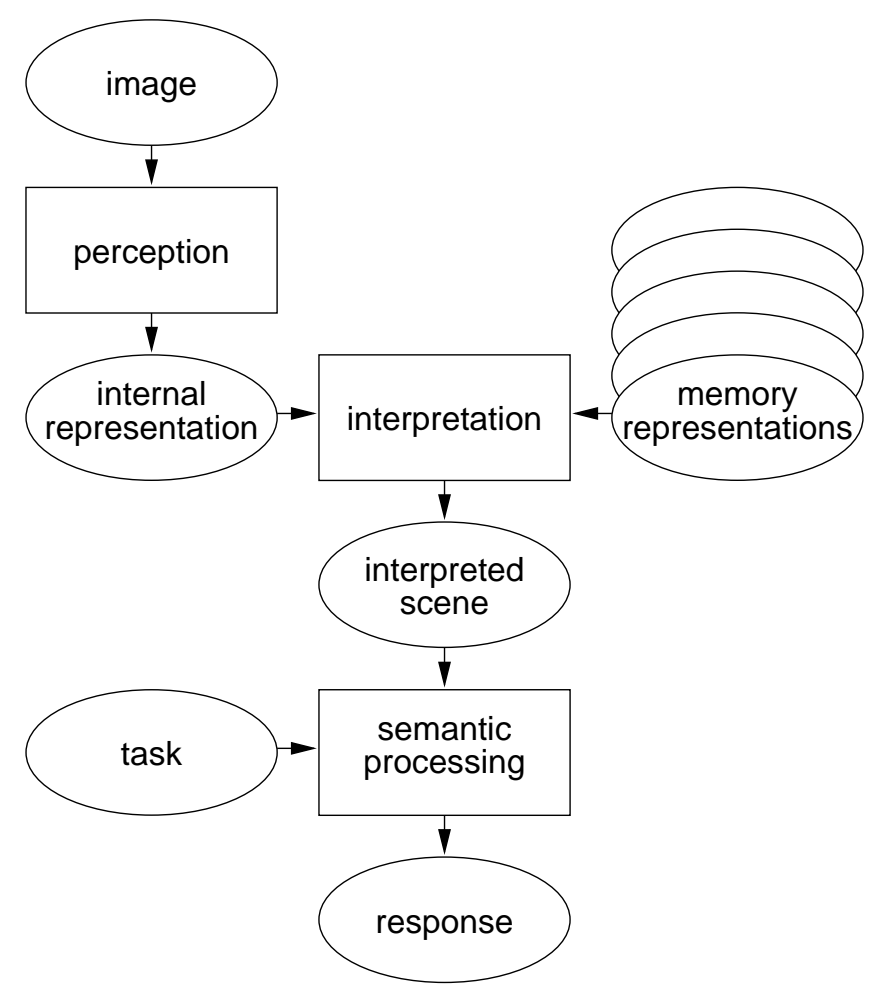

Figure 2.1: A diagrammatical depiction of visuo-cognitive processing of images. In this diagram, ellipses denote representations of information, and rectangles denote processes transforming one representation into another. Note that we consider the "response" to be a formulated sequence of actions, not its manifestation in terms of motoric events. 
between prototypical and non-prototypical object characteristics. Knowledge of reality therefore may be thought of as accumulated knowledge of (the behaviour of) outside world statistics. Instead of referring to a match between the internal representation and a memory representation, we will henceforth more appropriately refer to a match between the internal representation and knowledge of reality.

\subsubsection{Naturalness, usefulness and quality}

Given the visuo-cognitive processes as outlined above, we now ask how a successful interpretation of an image can be secured best. Returning to figure 2.1, we may readily conclude that for a successful interpretation of an image, the interpretation process should result in a satisfactory match between the internal representation and knowledge of reality. We therefore quite directly arrive at two principal requirements that an image of "good" quality should satisfy: (1) the internal representation of the image should be sufficiently precise; and (2) the degree of correspondence between the internal representation and knowledge of reality as stored in memory should be high.

We are now able to formalise the preceding discussion by defining (1) the usefulness of an image to be the precision ${ }^{3}$ of the internal representation of the image; and (2) the naturalness of an image to be the degree of correspondence between the internal representation of the image and knowledge of reality as stored in memory. Using these definitions, we define the quality of an image to be the degree to which the image is both useful and natural.

The sets of requirements that one needs to impose upon an image in order to maximise the usefulness or the naturalness of this image will in general not coincide. For example, detection or discrimination of objects in an image may require "exaggeration" of certain features of this image, resulting in a less natural reproduction of the image. Therefore, given the above definition of image quality, we postulate that the quality of an image will be given by a compromise resulting from simultaneously evaluating to what degree the image satisfies the sets of requirements that lead to maximising the usefulness or the naturalness of the image.

\footnotetext{
${ }^{3}$ We use the term precision here to refer to a kind of internal signal-to-noise ratio. Precision therefore does not necessarily mean a one-to-one correspondence with reality.
} 


\subsection{Experiments}

\subsubsection{Experiment 1: Influences of naturalness and usefulness on image quality}

Aim

In order to test the semantic description of image quality as given above, we performed an experiment allowing us to measure the influences of naturalness and usefulness upon image quality. To this end, we selected two kinds of manipulation, that is, varying the colour temperature of the reference white ${ }^{4}$ and varying chroma ${ }^{5}$ (Hunt 1992), which we expected to affect naturalness and usefulness in distinctly different ways. The first manipulation was expected to influence only the naturalness of the image, whereas the second manipulation was expected to influence both the naturalness and the usefulness of the image.

\section{Description}

The experiment, similar to experiments described by Fedorovskaya, de Ridder \& Blommaert (1997), de Ridder, Fedorovskaya \& Blommaert (1993), and de Ridder (1996), was performed using four colour images of natural scenes taken from a Kodak Photo CD. The colour temperature of the reference white was varied between $4650 \mathrm{~K}$ and $10300 \mathrm{~K}$, 6500K being the original, in seven steps of perceptually equal size, and chroma was scaled by a constant ranging from 0.5 to 2.0 in seven steps.

Seven subjects participated in the experiment. In three separate sessions they were shown on a CRT (cathode ray tube) the complete set of images, in random order, with three replications. In the first session, subjects' task was to judge the quality ${ }^{6}$ of the images, in the second session to judge the colourfulness ${ }^{7}$ of the images, and in the third session to judge the nat-

\footnotetext{
${ }^{4}$ Defined as "the temperature of a Planckian radiator whose radiation has the same chromaticity as that of a given stimulus". Unit: Kelvin, K.

${ }^{5}$ Defined as "the colourfulness of an area judged as a proportion of the brightness of a similarly illuminated area that appears to be white or highly transmitting". In the experiments $C_{u v}^{*}$, a correlate of chroma in the CIELUV colour space, was scaled.

${ }^{6}$ Defined as "the degree to which you like the colours in the image".

${ }^{7}$ Defined as "the presence and vividness of the colours in the image".
} 
uralness ${ }^{8}$ of the images. Subjects were instructed to use an eleven-point numerical scale ranging from 0 ("bad" or "weak") to 10 ("excellent" or "strong").

\section{Results}

Colourfulness judgments (averaged over subjects and scenes) versus chroma (diamonds) and colour temperature of the reference white (triangles) are shown in figure 2.2. The effect of scaling chroma on judged colourfulness is clearly visible. Less clear, although still significant, is the influence of colour temperature of the reference white on judged colourfulness; images are judged less colourful for higher temperatures of the reference white.

Quality judgments (diamonds) and naturalness judgments (triangles) versus colourfulness judgments, all averaged over subjects and scenes, are shown in figure 2.3 for the conditions chroma (solid curves) and colour temperature of the reference white (dashed curves). The figure shows that, as expected, quality correlates well with naturalness, although for the condition chroma the curve for quality is shifted with respect to the curve for naturalness towards higher values of colourfulness (and hence towards higher values of chroma). Note that a similar shift does not occur for the condition colour temperature of the reference white. This seems to dismiss the possibility of a straightforward "preference" for images with higher colourfulness.

Quality judgments versus naturalness judgments, depicted in figure 2.4, again show that the quality-naturalness curve for the condition chroma deviates significantly from a linear relation. The curve has a U-formed shape resulting from the abovementioned shift between quality judgments and naturalness judgments.

\section{Interpretation}

The first-order effect, that is, the high correlation $(r=0.93)$ between naturalness judgments and quality judgments for both conditions, can readily be interpreted in terms of the outlined semantic theory of image quality. In order to interpret the second-order effect, that is, the shift between naturalness judgments and quality judgments for the condition chroma, we adopt

${ }^{8}$ Defined as "the degree to which the colours in the image seem realistic to you". 


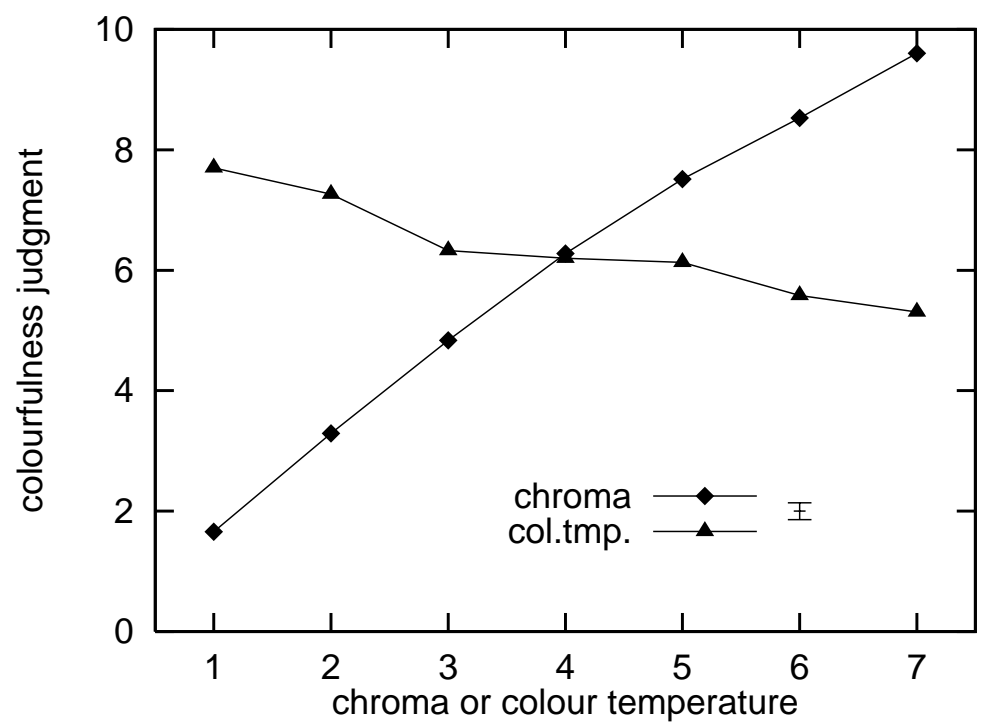

Figure 2.2: Colourfulness judgments (averaged over subjects and scenes) for the conditions chroma (diamonds) and colour temperature of the reference white (triangles). The error-bar denotes a distance of two average standard errors in the mean. The numbers 1-7 on the horizontal axis denote, for chroma, scaling by $0.50,0.63,0.79,1.00,1.26,1.59$ and 2.00 , and for the reference white a colour temperature of $4650 \mathrm{~K}, 5150 \mathrm{~K}, 5800 \mathrm{~K}, 6500 \mathrm{~K}$, $7400 \mathrm{~K}, 8650 \mathrm{~K}$ and $10300 \mathrm{~K}$. 


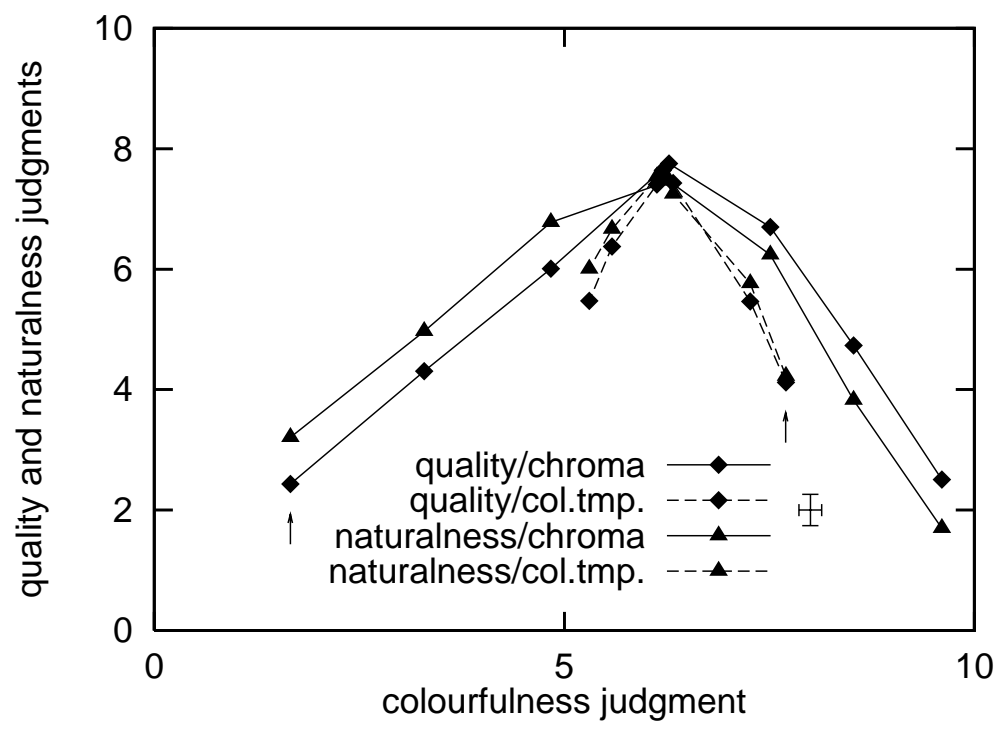

Figure 2.3: Quality judgments (diamonds) and naturalness judgments (triangles) versus colourfulness judgments (all averaged over subjects and scenes) for the conditions chroma (solid lines) and colour temperature of the reference white (dashed curves). The error-cross denotes a distance of two average standard errors in the mean, and the arrows denote the images with lowest chroma and lowest colour temperature of the reference white, respectively. 


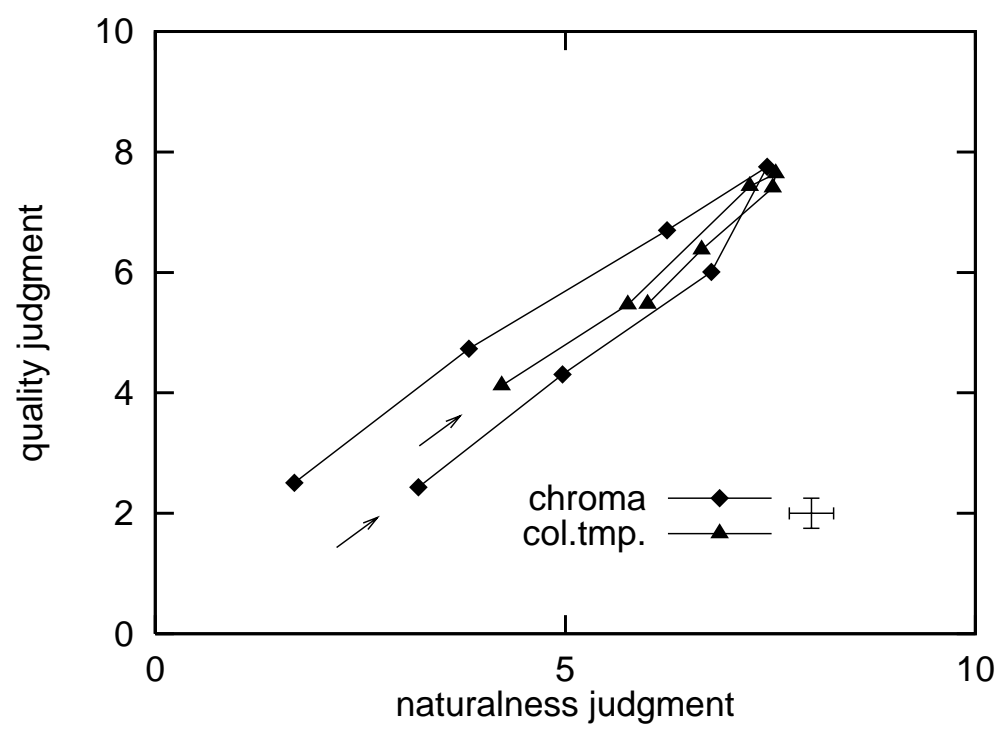

Figure 2.4: Quality judgments versus naturalness judgments (both averaged over subjects and scenes) for the conditions chroma (diamonds) and colour temperature of the reference white (triangles). The error-cross denotes a distance of two average standard errors in the mean, and the arrows denote the images with lowest chroma and lowest colour temperature of the reference white, respectively. 
the CIELUV colour space (recommended in 1979 by the Commission Internationale de l' Éclairage) as an appropriate, perceptually uniform colour space.

In CIELUV, the image can be thought of as a "cloud of dots", each dot corresponding to one pixel in the image. Scaling chroma can be described as a radial contraction or expansion of the cloud of dots towards or away from the reference white, while changing the colour temperature of the reference white can be described by a displacement of the entire cloud along the yellow-blue direction. The relevant difference between the two manipulations follows quite directly from their descriptions in CIELUV: changing chroma results in increased or decreased distances in colour space between any pair of dots of which the members do not represent exactly the same colour, while changing the colour temperature of the reference white has no effect on these distances.

Contrary to manipulations which preserve distances in colour space, manipulations which do affect distances in colour space will also affect the precision with which the image can be represented internally (since at a presumed, constant level of internal noise, affecting distances in a perceptually uniform space is equivalent to affecting an internal "signal-to-noise ratio"), and hence the usefulness of the image. The above discussion can therefore be concluded as follows: manipulations which do not affect the usefulness of an image, for example, changing the colour temperature of the reference white, will have approximately identical parameter settings for optimising the naturalness and the quality of the image. Manipulations which do affect the usefulness of an image, for example, changing chroma, will have different parameter settings for optimising the naturalness or the quality of an image. In the latter situation, the parameter settings optimising quality will tend to deviate with respect to those optimising naturalness towards values which increase the usefulness of the image.

\subsubsection{Experiment 2: Image quality regarded as a compro- mise between naturalness and usefulness}

Aim

In order to test the description of image quality in terms of a compromise between naturalness and usefulness, we devised an experiment in which 
we manipulated the brightness contrast of black-and-white images of natural scenes. Assuming that (1) usefulness is linearly related to perceived brightness contrast; and (2) the compromise can be adequately described by a linear combination of naturalness and usefulness, we may write image quality $Q$ in terms of naturalness $N$ and brightness contrast $C$ as:

$$
Q=\lambda_{1} N+\lambda_{2} C+\lambda_{3}
$$

and fit the vector $\vec{\lambda}$ to subjects' judgments of quality, naturalness and contrast as obtained in the experiment.

\section{Description}

The experiment was performed using four black-and-white images of natural scenes, obtained by transforming images from a Kodak Photo CD to the CIELUV colour space and setting $u^{*}$ and $v^{*}$ to zero. We then applied the global, pixel-wise transformation:

$$
\begin{aligned}
L^{*^{\prime}}= & \left(\frac{L^{*}-L_{\min }^{*}}{L_{\text {ave }}^{*}-L_{\text {min }}^{*}}\right)^{\gamma}\left(L_{\text {ave }}^{*}-L_{\min }^{*}\right)+L_{\min }^{*} \\
& \quad \text { for } L_{\min }^{*} \leq L^{*} \leq L_{\text {ave }}^{*} \\
L^{*^{\prime}}= & \left(\frac{L_{\max }^{*}-L^{*}}{L_{\max }^{*}-L_{\text {ave }}^{*}}\right)^{\gamma}\left(L_{\text {ave }}^{*}-L_{\max }^{*}\right)+L_{\max }^{*} \\
& \text { for } L_{\text {ave }}^{*}<L^{*} \leq L_{\max }^{*},
\end{aligned}
$$

(where $L^{*}$ represents the original lightness value of a pixel, $L^{*^{\prime}}$ its new value, and the subscripts "min", "max" and "ave" indicate the minimum, maximum and average lightness values of the original image) on the image using for $\gamma$ the values $0.25,0.35,0.50,0.71,1.00,1.41,2.00,2.82$ and 4.00. Applying this transformation will, for $\gamma<1$ decrease, and for $\gamma>1$ increase the brightness contrast of the image. The minimum and maximum lightness of the image are not affected, while in general the average lightness will remain at approximately the same lightness value.

Eight subjects participated in the experiment. In three separate sessions they were shown on a CRT the complete set of images, in random order, with three replications. In the first session, subjects' task was to judge the 
quality ${ }^{9}$ of the image, in the second to judge the brightness contrast ${ }^{10}$ of the images, and in the third session to judge the naturalness ${ }^{11}$ of the images. Subjects were instructed to use an eleven-point numerical scale ranging from 0 ("bad" or "low") to 10 ("excellent" or "high").

\section{Results}

Contrast judgments (averaged over subjects and scenes) versus the parameter $\gamma$ are shown in figure 2.5. The figure shows that, as expected, contrast increases for increasing values of $\gamma$. Figure 2.6 shows quality judgments (diamonds, averaged over subjects and scenes) and naturalness judgments (triangles, also averaged) versus contrast judgments (also averaged). The curve for quality is shifted with respect to the curve for naturalness towards higher values of contrast; a result which is similar to the results for the condition chroma in experiment 1 . To conclude, figure 2.7 shows quality judgments versus naturalness judgments. The U-formed shape already found in experiment 1 is clearly visible.

\section{Interpretation}

We again find a high $(r=0.95)$ correlation between naturalness judgments and quality judgments, confirming that naturalness is a principal factor constituting image quality. The shift of the curve for quality with respect to the curve for naturalness towards higher values of contrast can readily be interpreted when realising that higher contrast allows for more accurate detection and localisation of edges in the image, and thus for a more precise internal representation of the image.

The least-squares fit of our model to the quality judgments obtained in the experiment is given by:

$$
\begin{aligned}
& Q=\lambda_{1} N+\lambda_{2} C+\lambda_{3} \\
& \vec{\lambda}=(0.90,0.25,-1.08) .
\end{aligned}
$$

Figure 2.8 shows this fit (circles), together with the quality (diamonds) and

\footnotetext{
${ }^{9}$ Defined as "the degree to which you like the image".

${ }^{10}$ Defined as "apparent light-density differences".

${ }^{11}$ Defined as "the degree to which the image seems realistic to you".
} 


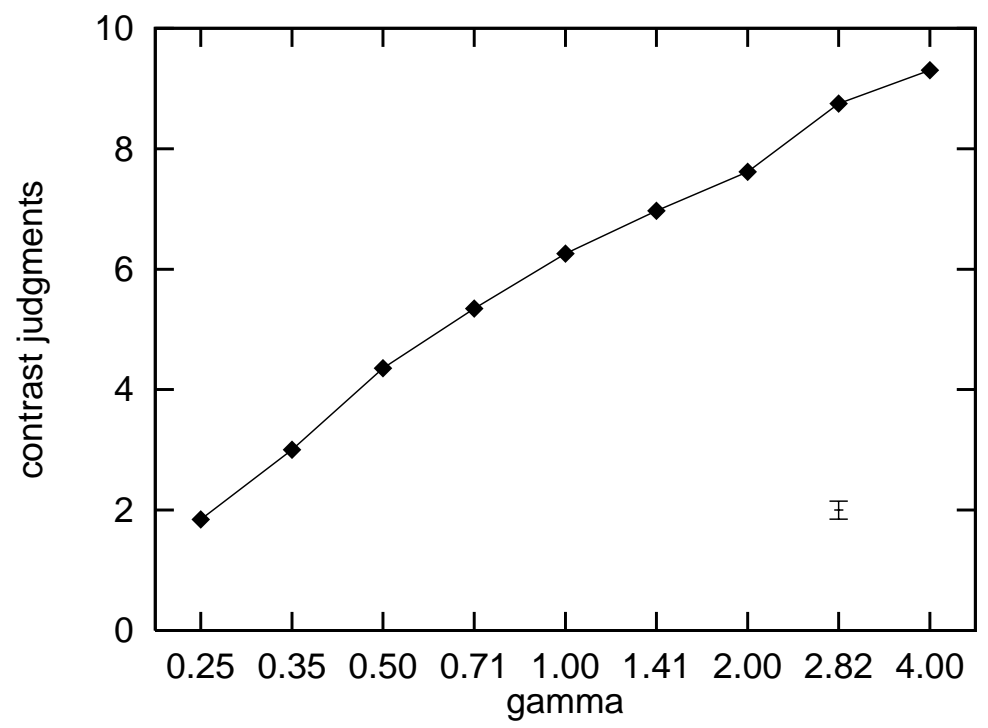

Figure 2.5: Contrast judgments (averaged over subjects and scenes) versus the parameter $\gamma$. The error-bar denotes a distance of two average standard errors in the mean. 


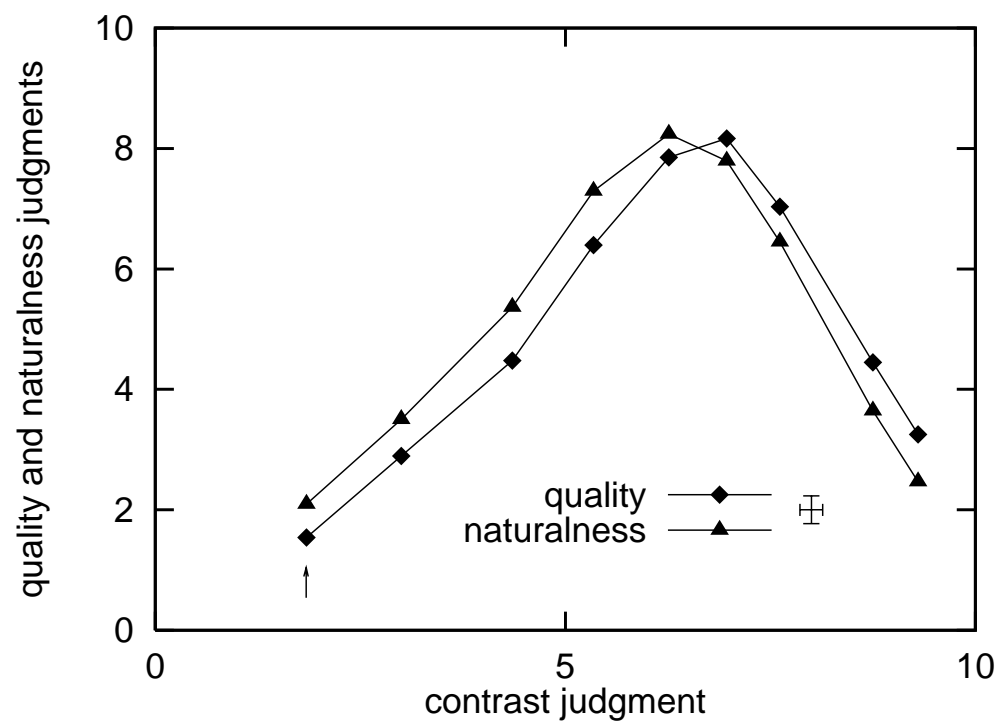

Figure 2.6: Quality judgments (diamonds) and naturalness judgments (triangles) versus contrast judgments (all averaged over subjects and scenes). The error cross denotes a distance of two average standard errors in the mean, and the arrow denotes the image with lowest $\gamma$. 


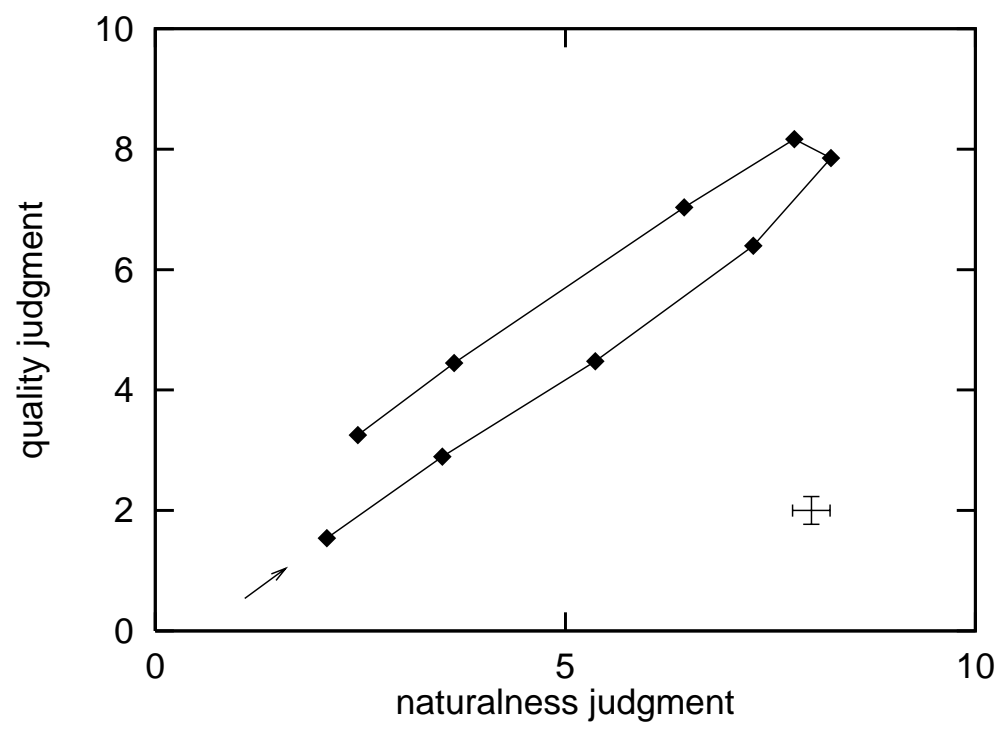

Figure 2.7: Quality judgments versus naturalness judgments (both averaged over subjects and scenes). The error-cross denotes a distance of two average standard errors in the mean, and the arrow denotes the image with lowest $\gamma$. 


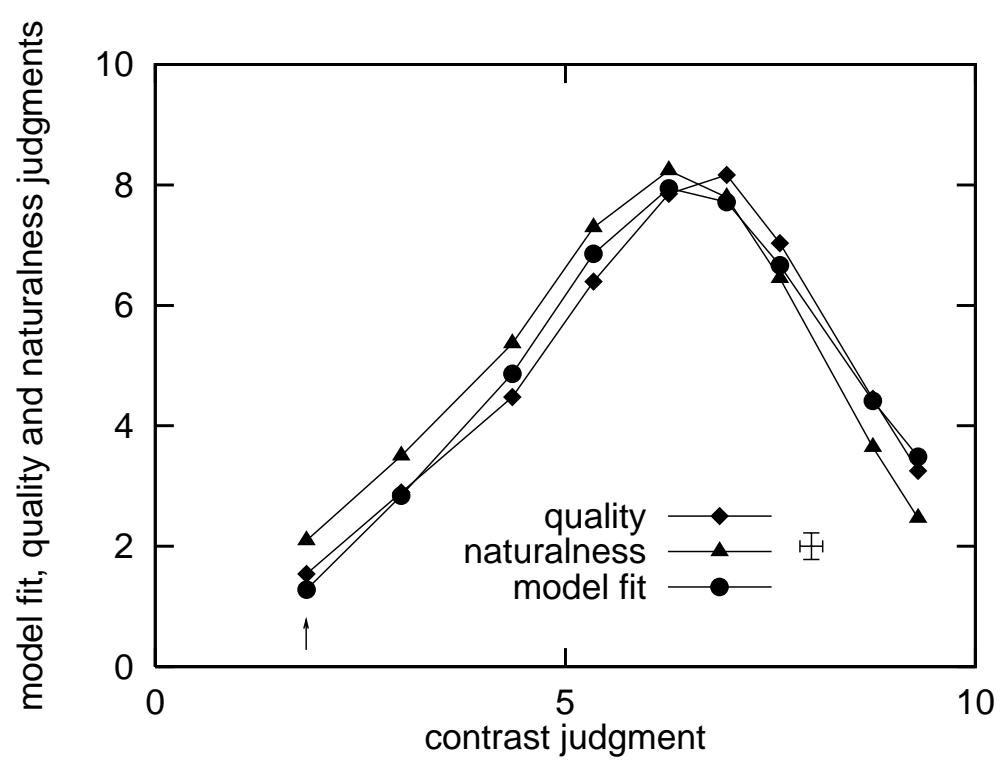

Figure 2.8: Quality judgments (diamonds) and naturalness judgments (triangles) versus contrast judgments (all averaged over subjects and scenes). The figure also shows the model fit (circles). The error cross denotes a distance of two average standard errors in the mean, and the arrow denotes the image with lowest $\gamma$.

naturalness (triangles) judgments. The correlation between the fit and the quality judgments is very high $(r=0.99)$. Considering (1) the primitive nature of the model; and (2) the strong non-linearity of the correlation coefficient as a measure for goodness-of-fit (that is, goodness-of-fit increases strongly for correlation $r$ approaching one), we may conclude that our description of image quality in terms of a compromise between naturalness and usefulness fits the data very well.

\subsection{Concluding remarks}

In pursuing a top-down, analytical approach, we have achieved a fundamental interpretation of the processes which play a role in the estimation of the attribute "quality" of an image. We have argued that image qual- 


\subsection{Concluding remarks}

ity is, to the observer, a useful attribute of an image, expressing how well the observer is able to employ the image as a source of information about the outside world; a view of image quality which is strikingly different from the "perceived distance to the original" philosophy often employed in image quality research.

The results of the experiments discussed in the previous sections support the concept developed here that the quality of an image can be described in terms of a compromise between the naturalness and the usefulness of that image. A logical next step to proceed from this point onwards would be to more thoroughly specify the naturalness and usefulness requirements imposed upon an image, for example, by means of formulating algorithms. Implementations of such algorithms will enable (1) the development of instrumental measures for the prediction of image quality; and (2) estimation of parameter settings optimising the quality of images.

At this point, two generalisations of the ideas discussed here may be interesting to note. First, our description of quality is essentially formulated independently of modality, suggesting (1) the possibility of simply applying the same ideas to, for example, the fields of sound or speech quality; and (2) the possibility to generalise the current description of image quality to a multi-modal semantic description of perceived quality of information presentation. It is highly likely that such a description will prove valuable in the design and evaluation of applications in which a multi-modal presentation of information plays a central role.

Second, in our description of image quality we have concentrated on the requirements imposed upon the information which is acquired from the environment. However, requirements ensuring a proper interaction between observer and environment should necessarily include requirements which ensure the ability to adequately respond to the environment. These requirements may then, in general, be imposed upon the means the observer is employing to control his environment. Such an approach is likely to result in a general theory of the quality of man-machine interaction ${ }^{12}$.

\footnotetext{
${ }^{12}$ See chapter 6 for a short discussion of two philosophical issues involved in this.
} 


\title{
Chapter 3
}

\section{Visual metrics: discriminative power through flexibility}

\begin{abstract}
An important stage in visual processing is the quantification of optical attributes of the outside world. We argue that the metrics used for this quantification are flexible, and that this flexibility is exploited to optimise the discriminative power of the metrics. We derive mathematical expressions for such optimal metrics and show that they exhibit properties resembling well-known visual phenomena. To conclude, we discuss some of the implications of flexible metrics for visual identification.
\end{abstract}

\subsection{Introduction}

Vision is often referred to as "inverse optics" (Poggio \& Koch 1985), that is, the process of measuring the characteristics of an optical image of the environment and reconstructing the material properties of this environment. Defined this way, vision involves a stage in which optical attributes of the outside world and the objects located within it are measured and internally quantified. Examples of such internally quantified measures are position, shape, size, texture, colour and brightness. The metrics used for this quantification will be the main topic of our discussion.

First, to clarify what we mean here by the term metric, we will consider

${ }^{0}$ This chapter is a slightly modified version of Janssen \& Blommaert (1999c). 
the measurement process. To enable the measurement of the strength of a certain attribute, an origin and a unit must first be selected. For example, to measure temperature one might select the melting point of water for the origin, and select one hundredth of the temperature difference between the boiling point and the melting point of water for the unit. Unit and origin together define a scale, in this case the Celsius temperature scale. Thus, to measure the temperature of an item one would take the temperature difference between the item and the origin of the scale and express this difference in the number of temperature units. The number obtained in this way is the Celsius scale value for this particular temperature.

In the above example, equal differences in the attribute strength will lead to equal differences in the scale value. Scales having this property are referred to as linear scales, since the relation between attribute strength and scale value is linear. Most familiar scales are of this type, however some well-known scales are not. Take, for example, the $d B$ scale for sound pressure or the $\mathrm{pH}$ scale for the degree of acidity. These scales are known as logarithmic scales, since their unit is not defined in terms of a difference but instead in terms of a ratio. For logarithmic scales, equal ratios in the attribute strength lead to equal differences in the scale value, and the relation between attribute strength and scale value therefore is logarithmic. For a certain scale, the exact relation between attribute strength and scale value is made explicit by the scale function. The scale function, together with the scale, the origin and the unit, defines what is called a metric (Watt 1989).

Standardised metrics as the Celsius, $d B$ or $p H$ metrics share one important and useful property: rigidity. Rigidity refers to the property that the scale function is uniquely defined and constant throughout time. For most measurements this property is essential. The Celsius temperature scale would be of little value when a temperature of $37^{\circ} \mathrm{C}$ as measured today would be different from a temperature of $37^{\circ} \mathrm{C}$ as measured tomorrow. At least, predicting tomorrow's temperature in terms of the Celsius temperature scale would make little sense. Rigidity of a metric allows for a unique specification of the attribute strength in terms of the scale value; $37^{\circ} \mathrm{C}$ is one and the same temperature, whenever and wherever you measure it.

We now return to vision and visual metrics. Traditionally, the view in visual research has been that visual metrics, such as the brightness metric, are essentially rigid (Weber 1846, Fechner 1860, Riesz 1933, Stevens 1957). Several exceptions to this rigidity are well known, for instance dark- and light-adaptation and crispening for the case of brightness, however each of these phenomena has traditionally been described and modelled sep- 
arately. As far as we know, there have been few attempts to unify these phenomena into one consistent description.

What we will try to do here is to follow an approach in which we will regard visual metrics no longer as being rigid. More specifically, we will assume that visual metrics are: (1) limited in range, that is, the scale has fixed lower and upper bounds; (2) limited in accuracy, that is, scale values cannot be represented with arbitrary precision due to the presence of noise; (3) intrinsically flexible (Blommaert 1995), which means that the scale function is allowed to vary in time; and (4) optimised with respect to overall discriminative power (Blommaert 1995, Watt 1989, Watt 1991), which means that the scale function is chosen such that the ability to discriminate between items in the outside world using the scale values of their measured attributes, is maximised.

We will divide our discussion of flexible metrics into four parts. First, we will consider the above four assumptions and discuss how and under what circumstances flexibility can be exploited to improve the discriminative power of a metric. Second, we will derive mathematical expressions for metrics satisfying the above assumptions. Third, using the obtained expressions we will explore some of the properties of these metrics, and relate these properties to well-known visual phenomena, namely brightness constancy and crispening. Last, we will consider some of the consequences of flexibility for the process of visual identification.

\subsection{The usefulness of flexibility}

We have assumed that visual metrics are limited in range and accuracy and, most importantly, that they are flexible and optimal with respect to discriminative power. The assumptions of limited range and limited accuracy seem straightforward, certainly when the constraints imposed by physical, or biological, implementations of these metrics are taken into account. Flexibility, however, is a less straightforward assumption. Flexibility may or may not prove useful, depending on the type of measurement which is performed. Noticeably, a measure requiring a strict one-to-one correspondence of the scale value with the attribute strength, as in the example of the temperature scale, will leave little space for flexibility of the metric.

The goal of early visual processing, however, is to extract the maximum 
possible amount of the information contained within the optical image, and to represent this information in a way which maximises its usefulness to the organism. Here, usefulness does not necessarily imply a one-toone correspondence with reality. Instead, a more meaningful criterion for usefulness often is the ability to discriminate between items in the outside world based on information about these items as it is represented internally. For example, the ability to discriminate between items on the basis of their colours may often be more important than establishing the exact colours of these items. For the aim of discriminative power, a one-to-one correspondence between internal representation and outside world is unnecessary. In fact, it is likely that such strict correspondence only decreases the ability to discriminate.

How can flexibility be exploited to improve discriminative power? To answer this question we will consider the assumptions of limited range and accuracy again. When scale values cannot be represented with arbitrary precision, for example due to the presence of noise, the ability to discriminate items in the outside world using their scale values will essentially be limited by the noise level. Items will only be discriminable when their scale values are at least in the order of one noise level apart and, assuming the noise level to be constant, discriminability will increase monotonically with scale value difference. Ultimately, to increase the overall discriminative power of a metric all scale value differences should be increased. Again assuming the noise level to be constant, this could be done by "stretching" the entire scale to a larger range. However, this range was assumed to be limited and constant.

Alternatively, overall discriminative power may be increased by locally stretching the scale, by means of locally increasing the derivative of the scale function, and by compressing the scale elsewhere. Such a mechanism allows for increased scale value differences while it simultaneously preserves the range of the metric. When the locations where the scale is stretched or compressed are carefully chosen, this may lead to a significant increase in overall discriminative power. This principle is illustrated in figure 3.1. The figure shows a simplified situation for the example of brightness (the internal measure) versus luminance (the attribute strength, given on a logarithmic scale). The situations for rigid and flexible metrics are shown in the left and right panel, respectively. The open circles on the luminance axes denote the luminances of a set of items under daylight illumination. The filled circles denote the luminances of the same set of items, this time under nocturnal illumination conditions. 

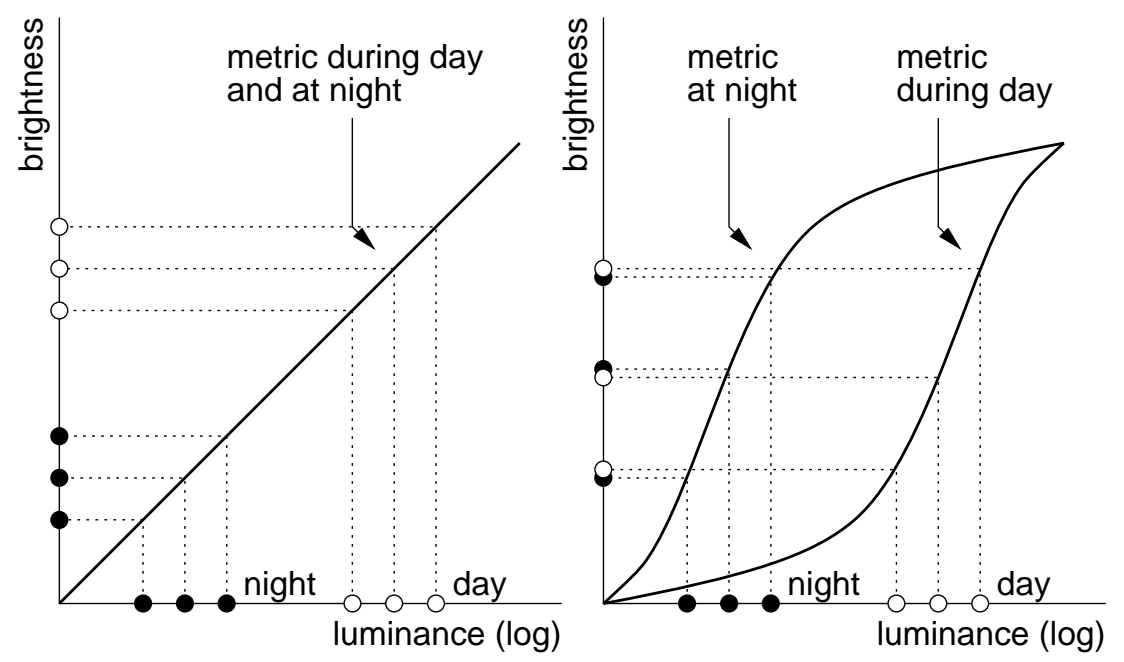

Figure 3.1: Rigid metric versus flexible metric. Simplified case for brightness (internal measure) versus luminance (physical attribute, on a logarithmic scale). Shown are the luminances and corresponding brightnesses of a set of items under daylight (open circles) and nocturnal (filled circles) illumination conditions. 
First, consider the left panel of figure 3.1. For a rigid metric to be able to represent all possible attribute strengths, the entire range of the attribute strength somehow has to be mapped onto the internal scale. For the case of luminance the range of the attribute strength is estimated to be ten orders of magnitude, however at any particular moment the luminance range found is typically only a small fraction of this entire possible range, usually only about three orders of magnitude (McCann 1988). Thus, even if the scale function of our rigid metric would be logarithmic, only about thirty percent of the metric would really be used at any particular moment. Boldly stated, this would be a waste of resources.

By locally stretching the scale we might increase all scale value differences by a factor of up to three, thereby significantly increasing overall discriminative power at any particular moment. However, to do this effectively, the locations where to stretch the scale need to depend on the current range of the attribute strength. Intuitively, the conclusion may already be that the amount of stretching of the scale must be closely related to the momentary distribution of the attribute strength, as shown in the right panel of figure 3.1. Here we encounter flexibility again, since in this case the scale function must be allowed to "follow" fluctuations in the momentary distribution of the attribute strength.

In the above discussion we have assumed the presence of noise, and it is therefore important to characterise the sources of this noise in more detail. First, we distinguish noise sources acting directly upon the attribute strength. For the case of luminance such a noise source would be photon noise. Second, we distinguish noise sources acting directly upon the scale value. When scale values are assumed to be encoded in neuronal impulse rates, random variability in these rates may be regarded as such a noise source. The first type of noise typically originates outside of the "measuring device", whereas the second type is generated by the measuring device itself. We will therefore refer to the first and second type of noise as external and internal noise, respectively. Figure 3.2 gives a graphical summary of this. Both internal and external types of noise can be regarded as compound noise, that is, each type can be thought to consist of a number of independent contributions from different sources. Usually, compound noise can be adequately modelled by assuming that the noise has Gaussian properties. This assumption will also facilitate the mathematical description in the next section, since Gaussian noise can be completely specified in terms of the two parameters mean and standard deviation.

Before we can start to derive mathematical expressions for optimal met- 


\subsection{Recipe for an optimal metric}

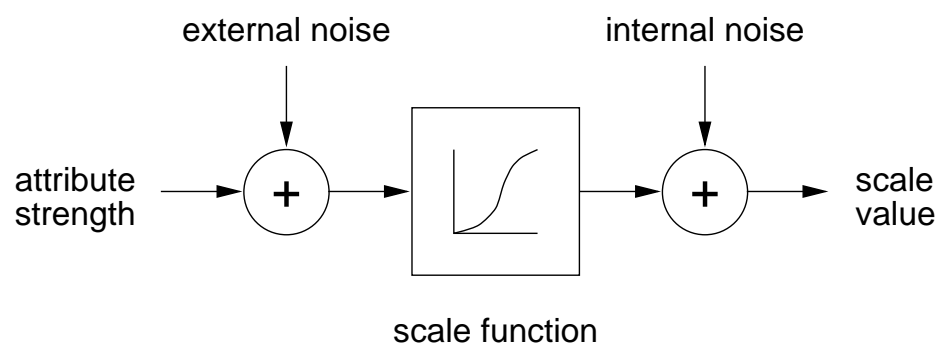

Figure 3.2: Block diagram showing the definitions we use here of external and internal noise.

rics, we will need to define a measure for overall discriminative power. The measure we will use here is the total number of topological errors made in the mapping of attribute strength onto scale value. Such topological errors occur when the ordering of a set of items by their scale value differs from the ordering of this same set of items by their attribute strength. The main justifications for this choice of measure are: (1) the number of topological errors is likely to be highly correlated with discriminative power, since when topological errors occur, discriminative power must be poor; and (2) the number of topological errors is a straightforward measure to calculate.

\subsection{Recipe for an optimal metric}

\subsubsection{Problem specification}

Assume that the values of the attribute strength $x$ (for example, luminance) are measured for a given set of $N$ items (an abstraction for the environment), and that the momentary distribution of the values of $x$ for this set of items is given by $\mu(x)^{1}$. Furthermore, assume that the measurement results are represented by scale values on an internal scale $s$ which is monotonic with $x$, that is, $s=s(x)$ and $d s(x) / d x \geq 0$ for all $x$, where $s(x)$ is the scale function. Notice that monotonicity is required here, since any deviations from monotonicity will inevitably result in topological errors being made in the mapping from $x$ to $s$. The momentary distribution of

\footnotetext{
${ }^{1}$ In this chapter, $\mu(x)$ is defined such that $\int_{X} \mu(x) d x=N$, where $X$ is the range of the attribute strength $x$ and $N$ is the number of items.
} 
the scale values on $s$ for a given distribution $\mu(x)$ of the attribute strengths and a given scale function $s(x)$ will be referred to as $\eta(s)$. If we want to optimise the scale $s$ such that overall discriminability of the items on $s$ is maximised, an obvious strategy here is to find the scale function $s(x)$ which minimises the number of topological errors.

The solution to this problem is as follows. Consider a small interval $d s$ on the scale $s$; the number of scale values in the interval $d s$ is then given by $\eta(s) d s$. When the probability of a topological error is written as a function of $s$, that is, $p_{e}=p_{e}(s)$, we may write the number of topological errors in the interval $d s$ as:

$$
N_{e, d s}=p_{e}(s) \eta(s) d s
$$

The total number of topological errors, $N_{e}$, is found by integrating this expression along the entire scale. Assuming that the range of the scale is given by $S$, we thus find:

$$
N_{e}=\int_{S} p_{e}(s) \eta(s) d s .
$$

Based on the assumption that the noise limiting the precision with which scale values can be represented is Gaussian, we show in appendix 3.6 that the probability of a topological error can be expressed as a monotonic function $^{2}$ of the total noise level $\sigma(s)$ times the item density $\eta(s)$ :

$$
p_{e}(s)=\frac{1}{2}-\frac{1}{2} \operatorname{erf} \frac{1}{2 \eta(s) \sigma(s)} .
$$

Assuming Gaussian noise propagation, the total noise level in equation 3.3 can be expressed in terms of the internal noise level $\sigma_{i}(s)$ and the external noise level $\sigma_{e}(x(s))$ as:

$$
\sigma(s)^{2}=\sigma_{i}(s)^{2}+\sigma_{e}(x(s))^{2}\left(\frac{d s(x)}{d x}\right)^{2}
$$

Finally, the link between $\eta(s)$ and $\mu(x)$ has to be made to solve the problem. This link follows from $s(x)$ being monotonic and the number of items being preserved, that is:

\footnotetext{
${ }^{2}$ Figure 4.4 in chapter 4 shows a plot of $p_{e}(s)$ versus $d / \sigma(s)$ (where $d=1 / \eta(s)$ ).
} 


$$
\eta(s) d s=\mu(x) d x .
$$

The optimal scale $s$ is found by substituting equations 3.3, 3.4 and 3.5 into equation 3.2 and minimising the resulting equation for $d s(x) / d x$.

Before trying to solve the set of equations 3.2-3.5, we will first consider an important consequence of equation 3.4. We have argued that locally stretching the scale can be used to increase discriminability. By regarding equation 3.4 we may investigate under what circumstances this mechanism will work. To this end, consider that the derivative of the scale function, $d s(x) / d x$, is used here as the instrument to increase the scale value difference $d s$ of two items with attribute strength difference $d x$ :

$$
d s=\left(\frac{d s(x)}{d x}\right) d x
$$

Discriminability of these two items will be determined by the ratio of scale value difference to the total noise level (SNR):

$$
\begin{aligned}
S N R & =\frac{d s}{\sigma(s)} \\
& =\frac{1}{\sigma(s)}\left(\frac{d s(x)}{d x}\right) d x .
\end{aligned}
$$

where $\sigma(s)$ is given by equation 3.4. We now distinguish between two extreme cases: (1) external noise dominant; and (2) internal noise dominant. For the first case, equation 3.4 can be approximated by:

$$
\sigma(s)=\sigma_{e}(x(s))\left(\frac{d s(x)}{d x}\right) .
$$

The ratio of scale value difference to total noise level then reduces to:

$$
S N R=\frac{d x}{\sigma_{e}(x(s))},
$$

which is independent of $d s(x) / d x$. When the influence of external noise is dominant, discriminability cannot be improved by stretching of the scale! For the second case, equation 3.4 can be approximated by: 


$$
\sigma(s)=\sigma_{i}(s),
$$

and equation 3.7 reduces to:

$$
S N R=\left(\frac{d s(x)}{d x}\right) \frac{d x}{\sigma_{i}(s)} .
$$

Here, SNR increases proportionally with the amount of stretching of the scale, and therefore stretching of the scale can indeed be used as a mechanism to improve discriminability. Having observed this, we will in the remainder of our discussion assume that the influence of internal noise is dominant, that is, we will assume that equation 3.4 can be approximated by equation 3.10. In some situations this assumption may not be true, for example at very low illumination levels where photon noise becomes important.

\subsubsection{Solution}

We start deriving the solution to our problem by regarding the Maclaurin series of the error function:

$$
\operatorname{erf} z=\frac{2}{\sqrt{\pi}} \sum_{m=0}^{\infty} \frac{z^{2 m+1}}{m !(2 m+1)} .
$$

Substituting $z=1 /(2 \eta(s) \sigma(s))$ in equation 3.12 and substituting the result in equation 3.3 , we get:

$$
p_{e}(s)=\frac{1}{2}-\frac{1}{\sqrt{\pi}} \sum_{m=0}^{\infty} \frac{(2 \eta(s) \sigma(s))^{-(2 m+1)}}{m !(2 m+1)} .
$$

Substituting the obtained expression for $p_{e}(s)$ in equation 3.2, we find for the total number of topological errors $N_{e}$ :

$$
\begin{aligned}
N_{e} & =\int_{S}\left(\frac{1}{2}-\frac{1}{\sqrt{\pi}} \sum_{m=0}^{\infty} \frac{(2 \eta(s) \sigma(s))^{-(2 m+1)}}{m !(2 m+1)}\right) \eta(s) d s \\
& =\frac{1}{2} \int_{S} \eta(s) d s-\frac{1}{\sqrt{\pi}} \sum_{m=0}^{\infty} \int_{S} \frac{(2 \eta(s) \sigma(s))^{-(2 m+1)} \eta(s)}{m !(2 m+1)} d s .
\end{aligned}
$$




\subsection{Recipe for an optimal metric}

The first term of equation 3.14 is constant, since $\eta(s)$ integrated over the range $S$ should always be equal to $N$, the number of items. As can be concluded from equation 3.14, the contribution $d N_{e}$ of each individual term in the sum of equation 3.14 to the total number of errors $N_{e}$ has the general form:

$$
d N_{e}=c_{n} \int_{S} \eta(s)^{n+1} \sigma(s)^{n} d s,
$$

where $n=-(2 m+1)$ and all $c_{n}$ are negative. We will first concentrate on finding a solution for this general form, and then infer the solution of equation 3.14 from it. When equation 3.5 is substituted in equation 3.15, the latter can be written as:

$$
d N_{e}=c_{n} \int_{X} \sigma(s(x))^{n} \mu(x)^{n+1}\left(\frac{d s(x)}{d x}\right)^{-n} d x,
$$

where $X$ is the range is the attribute strength. Assuming that internal and external noise are additive, the noise levels will be constant, that is, $\sigma_{i}(s)=$ $\sigma_{i}$ and $\sigma_{e}(x)=\sigma_{e}$. Using equation 3.4, we obtain:

$$
d N_{e}=c_{n} \int_{X}\left(\sigma_{i}^{2}+\left(\frac{d s(x)}{d x}\right)^{2} \sigma_{e}^{2}\right)^{n / 2} \mu(x)^{n+1}\left(\frac{d s(x)}{d x}\right)^{-n} d x,
$$

which should be minimised for $d s(x) / d x$. As discussed at the end of the previous section, we will look at the solution of equation 3.17 for the case that internal noise is dominant, that is, we assume that $\sigma_{i} \gg(d s(x) / d x) \sigma_{e}$ and approximate equation 3.4 by equation 3.10. For equation 3.17 we then find:

$$
\begin{aligned}
d N_{e} & =c_{n} \int_{X} \sigma_{i}^{n} \mu(x)^{n+1}\left(\frac{d s(x)}{d x}\right)^{-n} d x \\
& =c_{n} \sigma_{i}^{n} \int_{X} \mu(x)^{n+1}\left(\frac{d s(x)}{d x}\right)^{-n} d x
\end{aligned}
$$

since $\sigma_{i}$ was assumed to be constant. The problem of minimising equation 3.18 is an example of the general problem of finding the extremes of the integral: 


$$
J=\int_{x_{1}}^{x_{2}} \phi\left(x, y, y^{\prime}\right) d x
$$

where $y=y(x)$ is some function of $x, y^{\prime}=d y(x) / d x$ and $\phi$ is a function of the variables $x, y$ and $y^{\prime}$. The integral of equation 3.19 can be interpreted as a line integral along some path $\Gamma=y(x)$ joining the points $\left(x_{1}, y_{1}\right)$ and $\left(x_{2}, y_{2}\right)$, see, for example, Irving \& Mullineux (1959). Therefore, the problem is to find the path $\Gamma_{0}=y_{0}(x)$ which extremises the integral $J$. The solution to this problem is given by the Euler-Lagrange equation:

$$
\frac{\partial \phi}{\partial y}-\frac{d}{d x}\left(\frac{\partial \phi}{\partial y^{\prime}}\right)=0,
$$

which, when $\phi$ does not contain $y$ explicitly, reduces to:

$$
\frac{d}{d x}\left(\frac{\partial \phi}{\partial y^{\prime}}\right)=0 .
$$

Integrating equation 3.21 with respect to $x$ yields:

$$
\frac{\partial \phi}{\partial y^{\prime}}=k,
$$

where $k$ is a constant. After substitution of $y^{\prime}=d s(x) / d x$ and of $\phi=$ $\mu(x)^{n+1}(d s(x) / d x)^{-n}$ into equation 3.22 we obtain the solution to our problem:

$$
\frac{d s(x)}{d x}=k^{\prime} \mu(x) .
$$

By integrating equation 3.23 over the range $X$, it can easily be shown that the constant $k^{\prime}$ must be equal to $S / N$. Interestingly, the solution we have found is independent of the value of $n$, which means that we have found one solution which extremises every individual term in the sum of equation 3.14. The entire sum will therefore be extremised as well, and equation 3.23 represents the solution to the overall problem of minimising $N_{e}$ in equation 3.14.

The solution we have found here resembles histogram equalisation of $\mu(x)$ since, substituting equation 3.23 into equation 3.5: 


$$
\begin{aligned}
\eta(s) & =\mu(x)\left(\frac{d s(x)}{d x}\right)^{-1} \\
& =\text { constant. }
\end{aligned}
$$

Histogram equalisation is a well-known image processing tool used to increase image contrast or detectability of image features, see, for example, Ballard \& Brown (1982). The principal difference between the solution we find here and standard histogram equalisation is that here the characteristics of a flexible "measuring device" are adapted to the characteristics of the input signal, whereas in histogram equalisation the characteristics of the input signal are adapted to a supposedly rigid measuring device.

\subsubsection{Flexibility versus rigidity: performances compared}

How much can be gained by using flexible metrics? To obtain an impression of this, we used for the momentary distribution $\mu(x)$ the calculated luminance distributions of forty digitised images of natural scenes taken from a Kodak Photo $C D$, assuming that these images were to be displayed on a CRT (cathode ray tube) with $\gamma=2.5$. We then assumed three types of brightness metric: (1) a linear metric, that is, a metric of the type $s(x)=x / x_{0}$, where $x_{0}$ is an arbitrary reference value such that $x / x_{0} \leq 1 ;(2)$ a compressive metric, that is, a metric of the type $s(x)=(1+\beta)\left(x / x_{0}\right)^{1 / 3}-\beta$ (which for $\beta=0.16$ is proportional to CIE 1976 lightness $L^{*}$, see Hunt (1992)); (3) an optimal metric, that is, a metric according to equation 3.23. The optimal metric therefore varied from image to image, whereas the other metrics remained invariant. To facilitate a comparison of the performances of the three metric types, their ranges were chosen such that $s(x) \in S=[0,1]$ for all $x \in X=\left[0, x_{0}\right]$.

We then calculated, using equations 3.2, 3.3 and 3.5, for each image and each type of metric the number of topological errors $N_{e}$. To this end, we assumed that the total noise level $\sigma(s)$ is dominated by a constant internal noise level $\sigma_{i}$ equal to one percent of the range of the metric, that is, we assumed that $\sigma(s)=\sigma_{i}=0.01 S=0.01^{3}$. Furthermore, we scaled the distributions $\eta(s)$ by a factor one hundred, thereby assuming that each image contained exactly one hundred items to be discriminated.

\footnotetext{
${ }^{3}$ This assumption is consistent with a dynamic range of approximately 100 JND's (justnoticeable differences).
} 


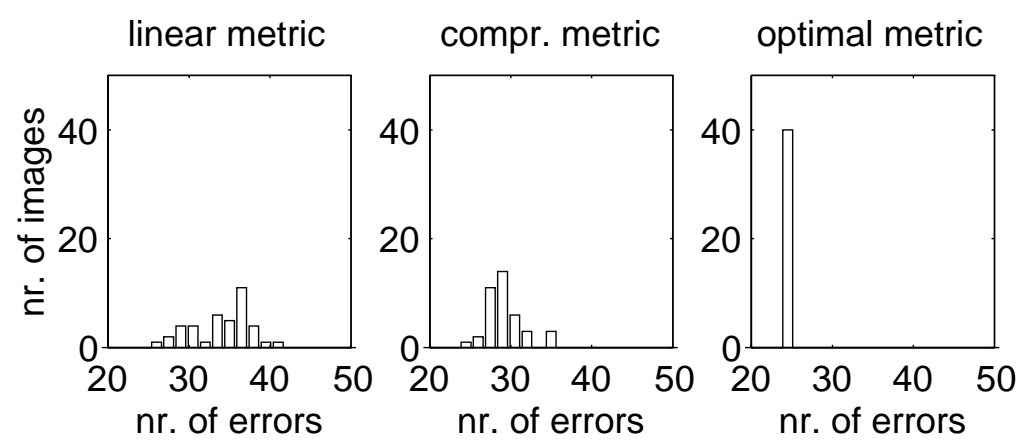

Figure 3.3: Discriminability, expressed in the number of topological errors, calculated for forty images of natural scenes and for three types of metric: linear, compressive and optimal.

Figure 3.3 shows frequency distributions of the number of topological errors for the linear, compressive and optimal metrics. Note that the number of topological errors for the optimal metric is the same for all images, something which is explained by $\eta(s)$ being constant and independent of $\mu(x)$ for optimal metrics. Figure 3.3 shows that, as expected, the optimal metric outperforms the two rigid metrics; the average number of topological errors for the linear, compressive and the optimal metrics is $34.0 \pm 3.8$, $29.3 \pm 2.2$ and 24.0 , respectively.

It is important to realise here that the above performance difference as calculated for digitised images is still relatively low, since the luminance ranges of the individual images are mapped into approximately the same range as a result of nearly optimal choices for diaphragm and exposure times when the images were taken. It is in everyday situations, where luminance ranges differ enormously, where the improved performance of flexible metrics becomes a distinct advantage.

\subsubsection{Concluding remarks}

Two important issues relating to the momentary distribution $\mu(x)$ of the attribute strength $x$ have so far not been addressed. The first issue is related to the influence of the factors time and location on $\mu(x)$. Consider, for example, the influence of time. According to the ideas we have forwarded, the scale function of a flexible metric should instantaneously follow any 


\subsection{Recipe for an optimal metric}

changes in the momentary distribution of the attribute strength. However, adaptation in real vision is known to be much faster when the illumination level increases then when the illumination level decreases. Although this asymmetry may simply be due to constraints in the biological implementation of the adaptation process, it may also serve to reduce the risk of physical damage resulting from exposure to high illumination levels.

The second issue is related to the concept of $\mu(x)$ itself, which is ill-defined in the context of real vision. We have introduced $\mu(x)$ as the momentary distribution of the attribute strength of "a set of items". The question arises what the correlate of $\mu(x)$ may be in real vision. Considering that flexibility can be most successfully exploited during the earliest stages of visual processing, where measures of attribute strength are least affected by the cumulative influence of internal noise, we may conclude that it would have to be a very early visual property.

Notwithstanding these issues, many well-known properties of vision fit in very well with the description of optimal visual metrics we have derived. For example, it can easily be shown that optimal metrics as specified by equation 3.23 exhibit properties which resemble phenomena such as dark- and light-adaptation, brightness constancy (Whittle 1994a), and crispening (Whittle 1994b). First, to demonstrate the case for brightness constancy, consider that when the momentary luminance distribution is given by $\mu(x)$, the brightness $s_{0}$ of an item with luminance $x_{0}$ is given by:

$$
\begin{aligned}
s_{0} & =\int_{-\infty}^{x_{0}} \frac{d s(x)}{d x} d x \\
& =\frac{S}{N} \int_{-\infty}^{x_{0}} \mu(x) d x
\end{aligned}
$$

When the illumination level changes by a factor $c$, all luminances will increase by this factor, since luminance is the product of illuminance and surface reflectance, and surface reflectance remains constant. We therefore find that the new luminance $x_{0}^{\prime}$ of our item will be equal to $c x_{0}$, and, in analogy, that the new luminance distribution $\mu^{\prime}(x)$ will be equal to $1 / c \cdot \mu(x / c)$. The new brightness $s_{0}^{\prime}$ of our item is given by:

$$
\begin{aligned}
s_{0}^{\prime} & =\frac{S}{N} \int_{-\infty}^{x_{0}^{\prime}} \mu^{\prime}(x) d x \\
& =\frac{S}{N} \int_{-\infty}^{c x_{0}} \frac{1}{c} \mu\left(\frac{x}{c}\right) d x
\end{aligned}
$$




$$
\begin{aligned}
& =\frac{S}{N} \int_{-\infty}^{x_{0}} \mu\left(x^{\prime}\right) d x^{\prime} \\
& =s_{0},
\end{aligned}
$$

that is, the brightness of our item is not influenced by the illumination level.

Second, to demonstrate the case for crispening, consider that for a small luminance difference $d x$ the corresponding brightness difference $d s$ is given by:

$$
\begin{aligned}
d s & =\left(\frac{d s(x)}{d x}\right) d x \\
& =\frac{S}{N} \mu(x) d x .
\end{aligned}
$$

The background luminance $x_{0}$ will usually appear in the luminance distribution $\mu(x)$ as a distinct peak for $x=x_{0}$. We therefore find that, for a constant luminance difference $d x$, the associated brightness difference $d s$ will also peak around $x=x_{0}$. In other words, we find that sensitivity for luminance differences is highest around the background luminance.

To conclude this discussion, we would like to stress that the above phenomena can now be understood as logical consequences of visual metrics having one essential property which itself is extremely useful: continuous optimisation of discriminative power by exploiting flexibility.

\subsection{Vision \& visual memory}

\subsubsection{Visual identification: vision versus memory}

The ability to discriminate between items based on their visual attributes is essential to any seeing organism. However, advanced vision is also characterised by the ability to identify or recognise items or item properties using past observations of these items. For this process we will assume the simple model shown in figure 3.4, in which identification is performed by comparing, or matching, scale values of measured item attributes with "standards" stored in memory. 


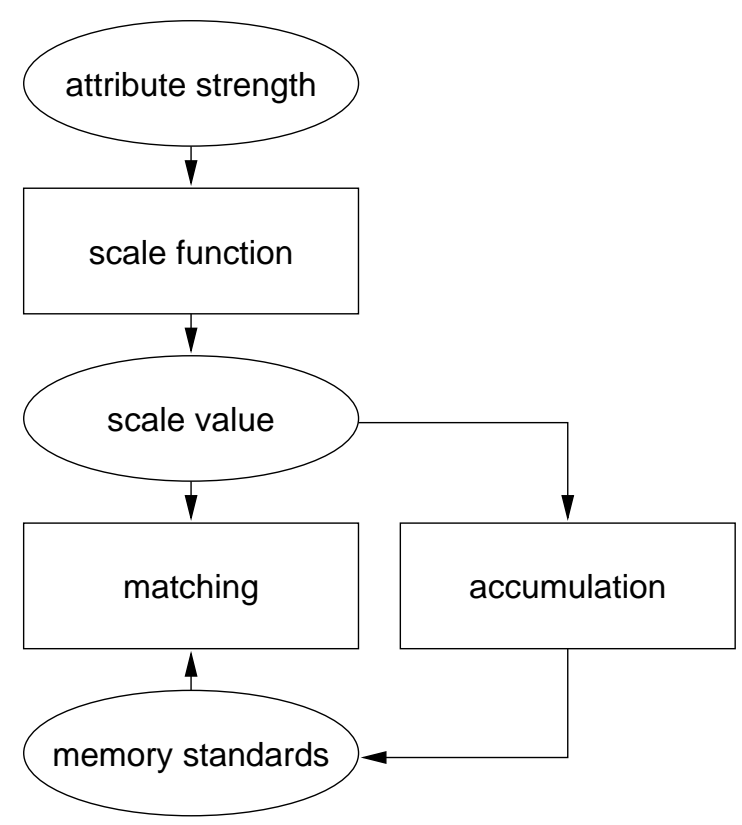

Figure 3.4: A simple model for the identification of items in the outside world. Identification is assumed to be performed by means of matching scale values with standards stored in memory. The memory standards themselves are assumed to be constructed by means of accumulation of scale values of past observations. 
The obvious way in which these standards are themselves constructed in memory is by the accumulation of past observations of the items. Such a long-term temporal integration will inevitably result in memory standards which are essentially rigid, at least when regarded on short-term intervals. This finding immediately raises an interesting question: how are observations, represented by scale values on a flexible visual metric, to be compared with standards which are essentially rigid?

\subsubsection{Calibrating visual metrics}

One way to cope with the above problem of comparing measurements represented on a flexible visual metric with rigid memory standards, is to "calibrate" the visual metric. Such calibration can be performed in several ways. We distinguish three possible calibration methods:

- First, the visual metric can be calibrated when the noise properties, or estimates of these, are known. Referring to equation 3.4, the metric can be found using:

$$
\left(\frac{d s(x)}{d x}\right)^{2}=\frac{\sigma(s)^{2}-\sigma_{i}(s)^{2}}{\sigma_{e}(x(s))^{2}} .
$$

- Second, the attributes of already recognised items can be used to estimate the current visual metric. This method explicitly requires that other item attributes be used to recognise these items first. For example, shape might be used to identify several items first, after which their "known" colours could be used to calibrate the metric for colour.

- Last, invariants in the momentary distribution of the attribute strength can be used to roughly estimate the current metric. Wellknown invariants include the grey-world assumption and the assumption that the brightest item is perfectly white. Other, more complex invariants may include various statistical assumptions about the momentary distribution of the attribute strength.

It is highly likely that other ways of calibrating the visual metric exist, including combinations of various calibration methods. However, another, perhaps more interesting situation occurs when the visual metric is left uncalibrated. 


\subsection{Vision \& visual memory}

\subsubsection{Uncalibrated visual metrics: partial flexibility}

The only information available for the accumulation and matching processes mentioned above is the measured attribute as represented by its scale value on the visual metric. When this metric is left uncalibrated, memory standards inevitably become fuzzy due to variability in the scale value as caused by the flexibility of the visual metric. Therefore, besides the difficulties which arise when this scale value is to be compared with memory standards, these memory standards themselves become less precise. It may therefore be useful to restrict the flexibility of the visual metric.

Assume that such restricted, or partial, flexibility can be formalised by introducing the eternal distribution $\mu_{e}(x)$, that is, the momentary distribution $\mu(x)$ integrated over a long-term interval. We can now introduce a weighting parameter $0 \leq \lambda \leq 1$ expressing the relative importances of $\mu(x)$ and $\mu_{e}(x)$ for the current visual metric $s(x)$. When equation 3.23 is generalised to:

$$
\frac{d s(x)}{d x}=\frac{S}{N}\left(\lambda \mu(x)+(1-\lambda) \mu_{e}(x)\right),
$$

$\lambda$ can be regarded as the degree of flexibility of the visual metric. The influence of flexibility on the variability in the scale value is now easily shown to be proportional to the degree of flexibility, since:

$$
\begin{aligned}
s_{0} & =\int_{-\infty}^{x_{0}} \frac{d s(x)}{d x} d x \\
& =\frac{S}{N} \int_{-\infty}^{x_{0}}\left(\lambda \mu(x)+(1-\lambda) \mu_{e}(x)\right) d x \\
& =\frac{S}{N} \lambda \int_{-\infty}^{x_{0}} \mu(x) d x+\frac{S}{N}(1-\lambda) \int_{-\infty}^{x_{0}} \mu_{e}(x) d x,
\end{aligned}
$$

where the first term represents the variability in the scale value $s_{0}$ for a given attribute strength $x_{0}$. This variability, together with variability in $x_{0}$ itself and together with the influences of external and internal noise, will be the main source of fuzziness of memory standards when the latter are assumed to be constructed by accumulation of scale values of past observations. Therefore, although partial flexibility will result in reduced discriminative power, memory standards will be less fuzzy and the comparison of scale values with these standards will be facilitated. The degree 
of flexibility of the visual metric then becomes the subject of optimisation in which both discriminability and identifiability play a role.

To conclude, the combination of an uncalibrated flexible metric with rigid memory standards should lead to contextual effects when what is observed is judged in relation to what is represented in memory. Interestingly, Yendrikhovskij, Blommaert \& de Ridder (1998) found such contextual effects. In a series of experiments, subjects were asked to judge the similarity of the object colour of a banana located on differently coloured backgrounds and displayed on a CRT to what they thought was the colour of a prototypical ripe banana. Subjects' judgments showed a significant influence of the colour of the background. This influence suggests that what is compared to the memory prototype is the apparent object colour, that is, the object colour as it is observed without correction for the colour of the surroundings. This is an important experimental result which seems to indicate that visual metrics are not calibrated.

\subsection{Conclusions}

We have shown that flexibility of a metric can be exploited to optimise the overall discriminative power of this metric. We have derived mathematical expressions for optimal metrics, and we have shown that such optimal metrics exhibit properties which correspond to well-known visual phenomena, such as brightness constancy and crispening. We have argued that these phenomena can therefore be understood as logical consequences of visual metrics being flexible and optimal with respect to discriminative power.

Furthermore, we have briefly investigated some of the consequences of flexible visual metrics for the process of visual identification. We have examined the problems which arise when observations represented on a flexible visual metric are compared to memory, and we have proposed two mechanisms for dealing with these problems. The first proposed mechanism is based on calibrating the visual metric. The second proposed mechanism leaves the visual metric uncalibrated and instead restricts the flexibility of the visual metric. 


\subsection{Probability of a topological error}

Consider the mapping from attribute strength $x$ to scale value $s$. Ideally, for two items $i$ and $j$ this mapping will be given by $s_{i}=s\left(x_{i}\right)$ and $s_{j}=s\left(x_{j}\right)$. However, due to the presence of noise we need a statistical description of this mapping:

$$
\begin{aligned}
& s_{i}=N\left(s\left(x_{i}\right), \sigma\left(s\left(x_{i}\right)\right)\right) \\
& s_{j}=N\left(s\left(x_{j}\right), \sigma\left(s\left(x_{j}\right)\right)\right),
\end{aligned}
$$

where $N$ is the normal probability density function, $x$ is the ideal noiseless attribute strength, $s(x)$ the scale function, and $\sigma(s)$ the total noise level. Now, if we assume that item $j$ is the successor of item $i$ in terms of ordering the items by attribute strength, we may use the approximations $x_{j}-x_{i}=1 / \mu(x), s\left(x_{j}\right)-s\left(x_{i}\right)=1 / \eta(s)$ and $\sigma\left(s\left(x_{i}\right)\right)=\sigma\left(s\left(x_{j}\right)\right)=\sigma(s)$, where $\mu(x)$ and $\eta(s)$ are the momentary distributions of attribute strength and associated scale value, respectively. We may now write the probability density function for the scale value difference $d s=s_{j}-s_{i}$ as:

$$
d s=N(1 / \eta(s), \sigma(s) \sqrt{2}) .
$$

A topological error occurs when item $j$ becomes the predecessor of item $i$ in terms of ordering the items by scale value, that is, when $d s<0$. To find the probability of a topological error, we must therefore integrate equation 3.32 from $d s=-\infty$ to $d s=0$ :

$$
p_{e}(s)=\int_{-\infty}^{0} N(1 / \eta(s), \sigma(s) \sqrt{2}) d(d s) .
$$

Using the linear transformation $u=(d s-1 / \eta(s)) / \sigma(s)$, this can be expressed as:

$$
p_{e}(s)=\int_{-\infty}^{-(\eta(s) \sigma(s) \sqrt{2})^{-1}} N(0,1) d u
$$

or, equivalently, as:

$$
p_{e}(s)=\frac{1}{2}-\frac{1}{2} \operatorname{erf} \frac{1}{2 \eta(s) \sigma(s)} .
$$




\title{
Chapter 4
}

\section{Predicting the usefulness and naturalness of colour reproductions}

\begin{abstract}
We present algorithms for predicting the usefulness and naturalness of colour reproductions of natural scenes. The algorithms are based on a computational model of the stages which lead to an observer's impression of the usefulness and naturalness of an image. These stages are (1) the perception, or internal quantification, of colour; (2) the construction of a memory standard for an object's colour based on its colour as observed in the past; and (3) matching of observed object colours with memory standards. In the first of the above stages, the internal quantification of colour, the concept of (partially) flexible metrics (chapter 3) plays a central role.

To test the usefulness algorithm, it was used to predict the discriminability of detail in black-and-white images of which the contrast was manipulated by applying an s-shaped transform on CIE 1976 lightness $L^{*}$. The naturalness algorithm was tested by using it to predict the naturalness of the grass, skin, or sky areas of images of which the colour was manipulated by shifting CIE 1976 hue angle $h_{u v}$ and scaling CIE 1976 saturation $s_{u v}$ of the grass, skin, or sky areas of the images. The predictions produced in these tests correspond quite well to experimentally obtained judgments of human subjects.
\end{abstract}

${ }^{0}$ This chapter is a slightly modified version of Janssen \& Blommaert (1999b). 


\section{Predicting the usefulness and naturalness of colour reproductions}

\subsection{Introduction}

Image quality is often considered in terms of a difference signal between the current, reproduced image and its "unprocessed" or "original" version. Well-known examples of this approach include JND (just-noticeable difference) maps (Daly 1993) and SQRI (square root integral) measures (Barten 1990), both of which are based on processing of the image and its original by implementations of visual front-end models and subsequent calculation of a difference measure from the two processed images. A serious drawback of this approach is that the fundamental question of what image quality is remains unanswered, as is the related question of what exactly the "original" of an image is. Thus, measures for differences between two versions of an image can be calculated, however what this tells about image quality remains unclear. Fitting the predictions of such models to experimentally obtained quality judgments of human subjects usually is the only means of attempting to make this translation.

We will follow a different approach here which does not suffer from this drawback. Essential to this approach is that we regard the quality of an image as the adequacy of this image as an input to visuo-cognitive information processing (chapter 2). The output of this visuo-cognitive processing determines in turn how well an observer is able to respond to occurrences in the outside world. Thus, in this view, the quality of an image becomes indeed a meaningful attribute of an image, telling how well the image can be employed as a source of information about the outside world.

When looking more closely at visuo-cognitive processing of images, see figure 4.1, we can discern three processing stages: (1) the construction of an internal representation of the image; (2) the interpretation of this representation by means of matching with representations stored in memory; and (3) semantic processing of the interpreted scene to formulate a proper response. For these stages to be completed successfully, the image should in general satisfy two main requirements: (1) the internal representation of the image should be precise; and (2) the match between the representation of the image and memory should be close. We refer to the degree to which an image satisfies these two requirements as the usefulness and the naturalness of the image, respectively.

Evidence for the appropriateness of the above description of image quality in terms of usefulness and naturalness was found in a series of experiments (see chapter 2). In these experiments, the separate influences of usefulness and naturalness on image quality were revealed by varying colour 


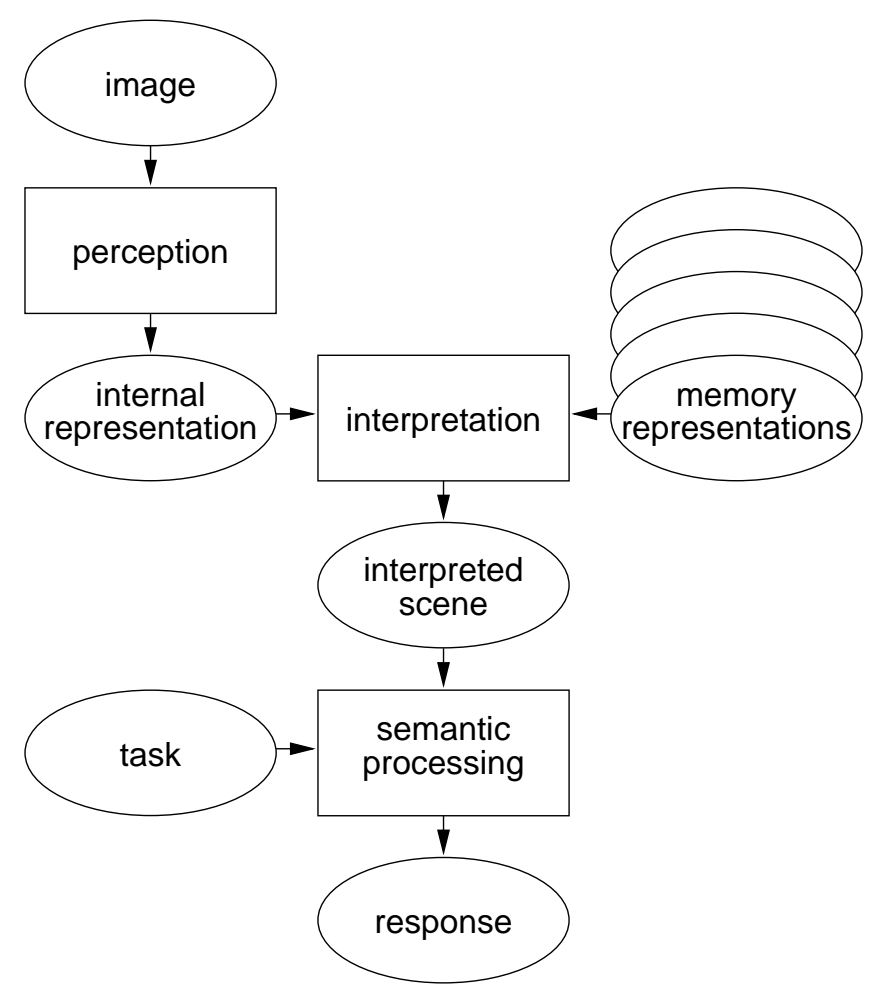

Figure 4.1: Visuo-cognitive processing of images. In this diagram, ellipses denote representations of information, and rectangles denote processes transforming one representation into another. 
temperature and chroma of a set of images of natural scenes and asking subjects to judge the quality and naturalness of the manipulated images. Manipulating colour temperature was expected to influence only the naturalness of the images, whereas manipulating chroma was expected to influence both naturalness and usefulness. Indeed, quality was found to be one-to-one related to naturalness when colour temperature was manipulated, whereas systematic differences between quality and naturalness were found when chroma was manipulated.

To predict the usefulness and naturalness of an image, we need to know what the internal representation of an image looks like. In other words, we need to know which attributes are represented and what metrics ${ }^{1}$ are used to quantify these attributes. Here, we will simply assume that the structure of the internal representation can be adequately described as a set of measured attributes such as position, shape, size, texture, brightness, and colour. When we focus on the attributes brightness and colour, as we will do in the remainder of this paper, we may conclude that the metrics used to quantify these attributes need not be rigid, that is, the scale function need not be constant over time. Moreover, when the scale function of these metrics is allowed to vary in time, this flexibility can be used to improve the discriminative power of the metric (Watt 1989, Watt 1991). Extending this idea, it is possible to find expressions for metrics which are optimal with respect to discriminative power (chapter 3). Interestingly, such optimal metrics exhibit properties resembling several well-known characteristics of colour vision, such as adaptation, crispening, and brightness- and colour-constancy.

Our aim here is to show how the ideas about visuo-cognitive processing of images, combined with the ideas about flexible metrics, can be used to implement algorithms for predicting the usefulness and naturalness of reproductions of colour images of natural scenes. The proposed usefulness algorithm is first presented with a set of images from which it calculates the luminance and chromaticity distributions of the pixels of the entire set of images, as well as the luminance and chromaticity distributions of the pixels of the image of interest. These distributions are used to calculate the (partially) flexible metrics upon which the brightness and colour distributions of the image of interest are represented. The algorithm then

\footnotetext{
${ }^{1} \mathrm{~A}$ metric is a system used for the quantification of measurements. A metric is defined by its origin, unit, scale, and scale function. Origin and unit together constitute the scale, upon which measurements of the strength of a certain attribute are represented by their scale values. The exact relation between attribute strength and corresponding scale value is given by the scale function.
} 
calculates overall discriminability from these distributions.

Besides the above steps, the naturalness algorithm also calculates the brightness and colour distributions of the areas of each image containing grass, (caucasian) skin, and sky. Averaged over the entire set of images, the brightness and colour distributions of the grass, skin, and sky areas represent the algorithm's "memory standards" for grass, skin, and sky. The algorithm calculates naturalness by comparing the brightness and colour distributions of the grass, skin, or sky areas of the image of interest with these memory standards. As we will show, the predictions produced by the usefulness and naturalness algorithms correspond well with experimentally obtained judgments of human subjects.

\subsection{Metrics for brightness and colour}

The concept of (partially) flexible metrics plays a central role in the algorithms we present here, and we will therefore start by introducing this concept. A metric is the instrument to quantify the measurement of an attribute's strength. The constituents of a metric are its scale, defined by an origin and a unit, and its scale function, which defines the relation between attribute strength and corresponding scale value (Watt 1989, Watt 1991). For physical (or biological) implementations of metrics, we may assume that the range of the metric is finite, that is, the scale has fixed lower and upper bounds, and that the precision with which scale values can be represented is limited, for example due to the presence of noise in the system which encodes the scale values. Therefore, when objects are to be discriminated by measurements of their attribute strengths, we find that the discriminative power is essentially determined by the ratio of scale value difference and noise level.

The central assumption we will make here is that the metrics used for the quantification of brightness and colour are optimal with respect to their discriminative power. Assuming a constant noise level, discriminative power can be increased by increasing scale value differences. One way to accomplish this would be to simply stretch the scale to a larger range. This, however, is no solution here since the range of the scale was assumed to be limited and constant. Another, slightly more complicated way to increase scale value differences is to locally stretch the scale there where an increase in discriminative power is desired, by locally increasing the derivative of the scale function, and to compress the scale elsewhere. In this way, the 

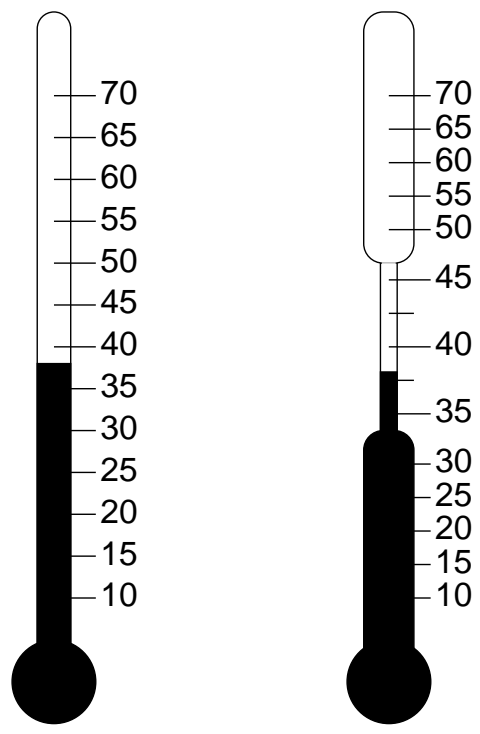

Figure 4.2: An example of how discriminative power can be increased by locally stretching the scale. For the thermometer, the scale function relates the attribute strength "temperature" to the measure "length of a mercury column". The exact relation between temperature and column length is determined by the amount of mercury in the reservoir and the diameter of the glass tube. For the thermometer at the right, discriminative power in the range $35^{\circ}$ to $45^{\circ}$ has been increased by locally reducing the diameter of the glass tube. The range of the scale, that is, the maximum length of the mercury column, has nevertheless been preserved by increasing the diameter of the glass tube elsewhere.

range of the scale can be preserved while scale value differences can be selectively increased. An example of this principle is shown in figure 4.2.

Extending this idea, it can be shown that to optimise overall discriminative power, the derivative of the scale function should be proportional to the momentary distribution of the attribute strength, as explained in chapter 3 . This result, which is found under the conditions of an internal noise level (due to, for example, random variability in neuronal response rates) which is constant and an external noise level (due to, for example, photon noise) which is negligibly small, can intuitively be understood by realising that to optimise overall discriminative power, the amount 
of stretching of the scale should be largest for those ranges of attribute strength which occur most frequently. Such a mechanism bears a close resemblance to the image processing tool known as histogram equalisation (Ballard \& Brown 1982) and, most importantly, requires the scale function to be flexible. Interestingly, such flexible, optimal metrics exhibit properties resembling some well-known visual phenomena, such as dark- and lightadaptation, crispening (Whittle 1994a, Whittle 1994b), and brightness- and colour-constancy.

The above type of metric is optimal with respect to discriminative power. However, for the aim of identification a metric must satisfy other, partially conflicting requirements. Most notably, identification requires that the scale function be rigid, to facilitate the comparison of what is observed at the present with what has been observed in the past. To satisfy this requirement and to simultaneously preserve discriminative power, the degree of flexibility of the metric must be restricted yet not reduced to zero. We will therefore use the concept of partially flexible metrics (chapter 3). Mathematically, such metrics can be specified by:

$$
\frac{d s(x)}{d x} \propto \lambda \mu(x)+(1-\lambda) \mu_{e}(x),
$$

where $x$ is the attribute strength, $s(x)$ the scale function, $\mu(x)$ the momentary distribution of the attribute strength ${ }^{2}, \mu_{e}(x)$ the "eternal" distribution of the attribute strength, and $0 \leq \lambda \leq 1$ the degree of flexibility of the metric. Here, the eternal distribution $\mu_{e}(x)$ can be thought of as the momentary distribution $\mu(x)$ integrated over a long-term interval.

Measurement usually involves a stage in which a sensor converts the attribute to be quantified into another attribute which is more accessible for measurement. For example, the thermometer of figure 4.2 converts the attribute "temperature" into the attribute "length of a mercury column" which can be measured easily. The receptors in the retina perform a similar task, by converting a complex spectrum of radiated or reflected energy into the set of three values we experience as a colour. These three values can be regarded as three separate dimensions, with each having its own metric assigned to it, which make up the attribute colour. In the algorithms we present here, we have chosen to use luminance $Y$ and CIE 1976 chromaticity coordinates $u^{\prime}$ and $v^{\prime}$ for these dimensions. We have

\footnotetext{
${ }^{2}$ In this chapter, $\mu(x)$ is defined such that $\int_{X} \mu(x) d x=1$, where $X$ is the range of the attribute strength $x$. Note that this definition differs from the one used in chapter 3 , where $\mu(x)$ was defined such that $\int_{X} \mu(x) d x=N$, where $N$ is the number of items.
} 


\section{Predicting the usefulness and naturalness of colour reproductions}

made this choice for two reasons: (1) the dimensions $Y, u^{\prime}$, and $v^{\prime}$ resemble the early-visual dimensions of colour, namely brightness, red-green, and yellow-blue; and (2) $Y, u^{\prime}$, and $v^{\prime}$ coordinates can be relatively easily calculated for digitised images displayed on a CRT (cathode ray tube).

In practice, to calculate what the metrics for $Y, u^{\prime}$, and $v^{\prime}$ look like for a particular image we therefore need to perform the following two steps. First, the momentary and eternal distributions $\mu$ and $\mu_{e}$ must be calculated separately for $Y, u^{\prime}$, and $v^{\prime}$. To model the eternal distribution $\mu_{e}$ we calculated the frequency distributions of the $Y, u^{\prime}$, and $v^{\prime}$ coordinates of the pixels of a set of 77 images of natural scenes taken from two Kodak photo CD's, and to model the momentary distribution $\mu$ we calculated the frequency distributions of the $Y, u^{\prime}$, and $v^{\prime}$ coordinates of the pixels of the image of interest. For these calculations we assumed that the images were to be displayed on a PAL (European colour television) compliant CRT with $\gamma$ corrected to the value 2.5. Second, the metrics for $Y, u^{\prime}$, and $v^{\prime}$ were calculated from the above distributions for a particular degree of flexibility $\lambda$ by calculating the weighted sum $\lambda \mu+(1-\lambda) \mu_{e}$ and integrating the result. Figure 4.3 summarises this procedure with an example.

\subsection{Predicting usefulness}

In the introduction, we have defined usefulness in terms of the degree of precision of the internal representation of an image. Here, precision does not necessarily imply that an item's attributes as they are represented internally should correspond one-to-one to the physical characteristics of the item. Instead, the ability to discriminate between items in the outside world on the basis of their internally quantified attributes is likely to be a more meaningful criterion for precision. In this section we will therefore define a measure for usefulness which is based on the idea that the usefulness of an image is essentially determined by the overall discriminability of the items in the image. An essential stage in the definition of such a measure is the internal quantification of attribute strength. For this we will use the concept of (partially) flexible metrics presented in the previous section, and limit ourselves to the attributes brightness and colour. 

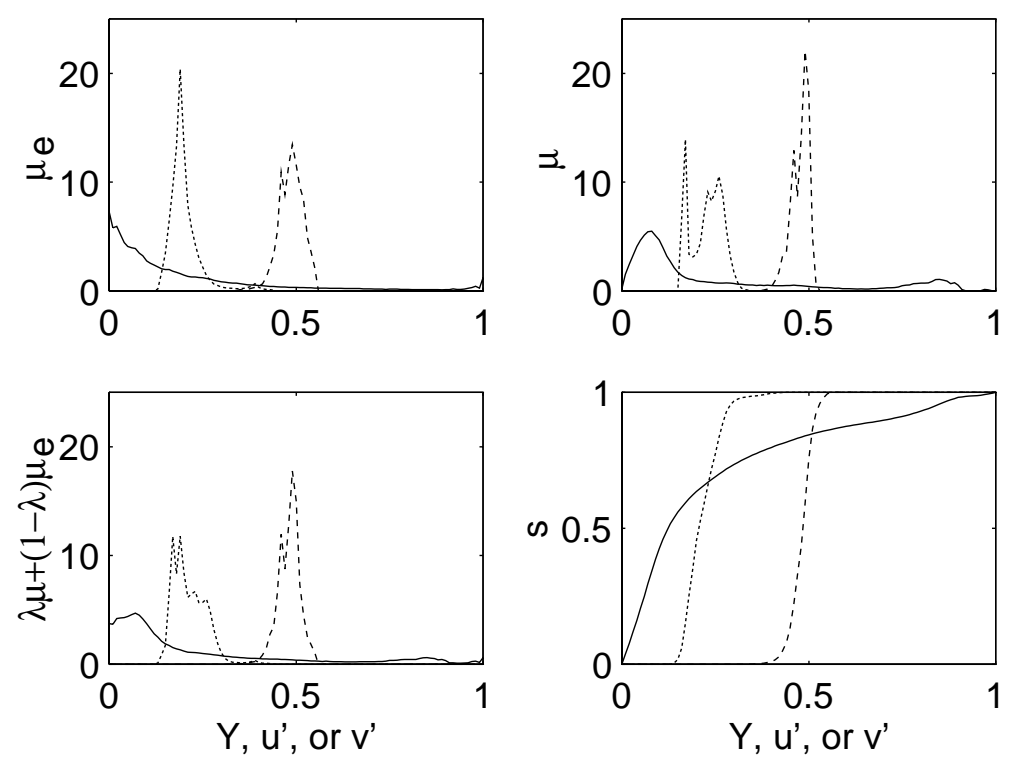

Figure 4.3: A calculated set of $\left(Y, u^{\prime}, v^{\prime}\right)$ metrics for an image. The upper left panel shows calculated $\left(Y, u^{\prime}, v^{\prime}\right)$ frequency distributions for the entire set of 77 images, representing the eternal distributions. The upper right panel shows calculated $\left(Y, u^{\prime}, v^{\prime}\right)$ frequency distributions for one particular image, representing the momentary distributions. The lower left panel shows the weighted sum of momentary and eternal distributions for $\lambda=$ 0.5 . Integrating these distributions yields the set of metrics for $Y, u^{\prime}$, and $v^{\prime}$ for this image. Note that the ranges of $u^{\prime}$ and $v^{\prime}$ on the horizontal axes are set to $[0,1]$ and that the range of $Y$ is normalised to fit in this range. Plots for $Y$ are drawn with solid lines, those for $u^{\prime}$ with dotted lines, and those for $v^{\prime}$ with dashed lines. 


\subsubsection{Discriminability}

In the previous section we have assumed that scale values can only be represented with a limited precision, for example due to the presence of noise in the system encoding these scale values. Discriminability of two items by their measured attribute strengths will therefore essentially be determined by (1) the scale value difference between the items; and (2) the noise level. The measure for discriminability which we will use here is the probability of a topological error. Topological errors occur when the ordering of a set of items by their scale values differs from the ordering of the same set of items by their physical attribute strengths, and the occurrence of topological errors therefore is a strong indicator that discriminability is poor. Assuming Gaussian noise properties, the probability of a topological error, $p_{\text {err }}$, can be shown (see chapter 3 ) to be given by:

$$
p_{\text {err }}=\frac{1}{2}-\frac{1}{2} \operatorname{erf} \frac{d}{2 \sigma},
$$

where $d$ is the ideal, noiseless scale value difference between any pair of items and $\sigma$ the noise level. In figure 4.4, $p_{e r r}$ is plotted versus $d / \sigma$ on a logarithmic scale. The figure shows that $p_{\text {err }}$ increases asymptotically to a maximum value of 0.5 when $d / \sigma$ approaches zero, and that $p_{\text {err }}$ remains essentially constant when $d / \sigma$ decreases below $10^{-1}$ or when $d / \sigma$ increases beyond $10^{1}$.

When the attribute strength distribution of the set of items is given by $\mu(x)$, the associated scale value distribution $\eta(s)$ on the metric $s(x)$ will be given by:

$$
\eta(s)=\mu(x)\left(\frac{d s(x)}{d x}\right)^{-1},
$$

where $d s(x) / d x$ is the derivative of the scale function, which for a (partially) flexible metric is given by equation 4.1. The probability of a topological error will be highest for neighbouring items. For neighbouring items on the metric, the scale value difference $d$ will in close approximation be given by $1 / N \eta(s)$, where $N$ is the number of items in the set. We may therefore approximate equation 4.2 by:

$$
p_{e r r}(s)=\frac{1}{2}-\frac{1}{2} \operatorname{erf} \frac{1}{2 N \eta(s) \sigma(s)} .
$$




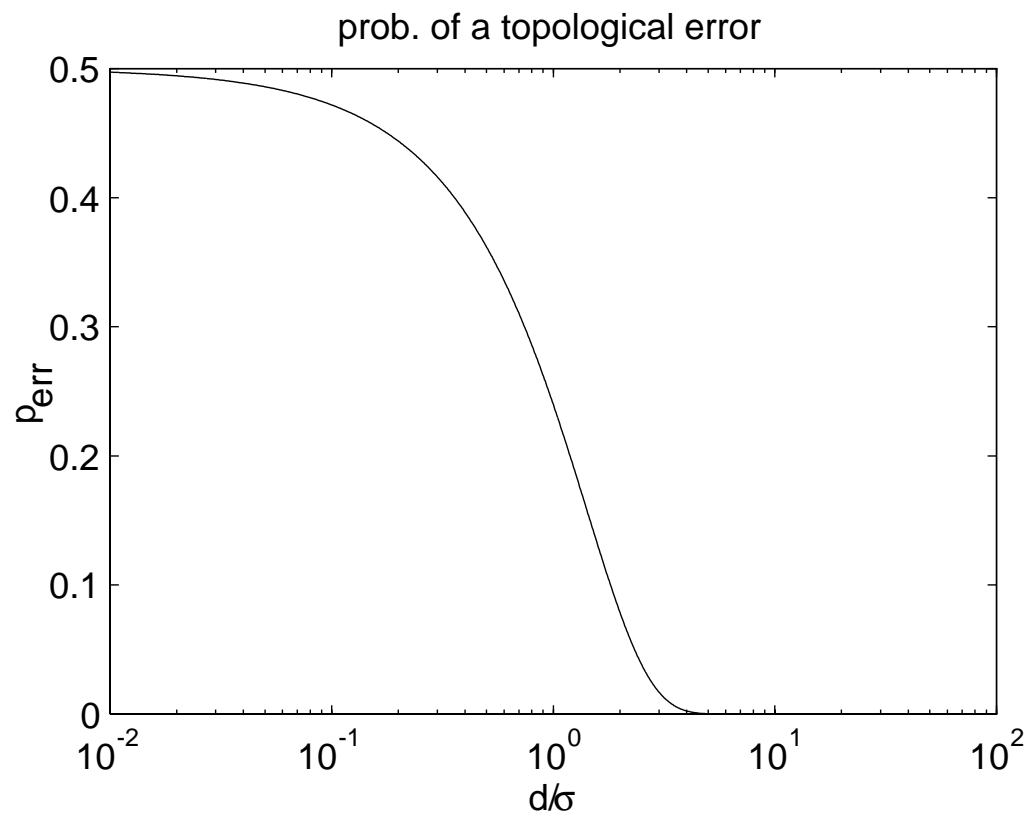

Figure 4.4: The probability of a topological error, $p_{e r r}$, versus the ratio of scale value distance $d$ and noise level $\sigma$ (on a logarithmic scale). 
To obtain the overall probability of a topological error, we must integrate this expression along the entire range $S$ of the metric:

$$
P_{\text {err }}=\int_{S} p_{e r r}(s) \eta(s) d s
$$

The measure $P_{\text {err }}$ must be calculated for each dimension separately. For multi-dimensional attributes like colour, overall discriminability $D$ must somehow be derived from the values found for $P_{e r r}$ along the $n$ individual dimensions. Since there is no obvious way in which this can be done, we will decrease the set of possible solutions by imposing some desired characteristics. Assuming that overall discriminability $D$ is normalised to the range zero to one, these characteristics are: (1) when $P_{\text {err }}$ increases for one or more dimensions, then overall discriminability $D$ should decrease; (2) when $P_{e r r}=0$ (that is, discriminability is perfect) for at least one dimension, then overall discriminability $D=1$; and (3) when $P_{\text {err }}=1 / 2$ (that is, discriminability is poorest) for all $n$ dimensions, then discriminability $D=0$. Perhaps the simplest way to satisfy these characteristics is when:

$$
D=1-2^{n} \prod_{i=1}^{n} P_{e r r, i}
$$

To conclude, the measure we now have was derived for a set of $N$ items. Although for Mondrian-like images the individual patches composing such images may be regarded as the items, for the category of natural images it is unclear what exactly these items are and, therefore, what the value of $N$ is for a particular image. In our calculations we have made the arbitrary choice to set $N$ to the value one hundred. Furthermore, assuming approximately one hundred JND's (just-noticeable differences) along each dimension, we have set $\sigma$ to the value 0.01 and $S$ to the range [0,1]. For the ratio $d / \sigma$ these choices lead to an average value of exactly one, which in figure 4.4 lies in the centre of the interval where $P_{\text {err }}$ is most sensitive to changes in $d / \sigma$. In general, although absolute values found for $D$ will depend on the choices made for $N, S$, and $\sigma$, we have found that when ratios of $D$ values are used to compare different images or different versions of one image, results are quite robust to changes in $d / \sigma$ of up to one order of magnitude from the value we have used here.

In practice, calculation of the usefulness of an image can now be performed as follows. First, the metrics for $Y, u^{\prime}$, and $v^{\prime}$ must be calculated for the image following the procedure discussed in the previous section. 


\subsection{Predicting usefulness}

Next, using equation 4.3 , the scale value distributions $\eta(s)$ can be calculated from the momentary distributions $\mu(x)$ and the scale functions $s(x)$ for the dimensions $Y, u^{\prime}$, and $v^{\prime}$ separately. From the obtained scale value distributions, $P_{e r r}$ can then be calculated for the individual dimensions using equations 4.4 and 4.5. Finally, overall discriminability $D$ is found by substituting the obtained results in equation 4.6. Part of this procedure is summarised in figure 4.5 with an example.

\subsubsection{Results and discussion}

In this subsection we will compare predictions of the above algorithm with experimentally obtained judgments of human subjects. To this end, we manipulated the brightness contrast of four digitised black-and-white images of natural scenes by applying a pixel-wise, s-shaped transformation on CIE 1976 lightness $L^{*}$ :

$$
\begin{aligned}
L^{*^{\prime}}= & \left(\frac{L^{*}-L_{\min }^{*}}{L_{\text {ave }}^{*}-L_{\min }^{*}}\right)^{\gamma}\left(L_{\text {ave }}^{*}-L_{\min }^{*}\right)+L_{\min }^{*} \\
& \text { for } L_{\min }^{*} \leq L^{*} \leq L_{\text {ave }}^{*} \\
L^{*^{\prime}}= & \left(\frac{L_{\max }^{*}-L^{*}}{L_{\max }^{*}-L_{\text {ave }}^{*}}\right)^{\gamma}\left(L_{\text {ave }}^{*}-L_{\max }^{*}\right)+L_{\max }^{*} \\
& \quad \text { for } L_{\text {ave }}^{*}<L^{*} \leq L_{\max }^{*},
\end{aligned}
$$

where $L^{*}$ represents the original lightness of a pixel, $L^{*^{\prime}}$ the new value, and where $L_{\min }^{*}, L_{\max }^{*}$, and $L_{\text {ave }}^{*}$ represent the minimum, maximum, and average lightness of the pixels in the original image, respectively. Furthermore, the parameter $\gamma$ is specified in terms of a gain-factor $g$ as:

$$
\gamma=10^{g}
$$

The transformation is shown in figure 4.6. Applying this transformation will, for $g<0$, decrease the brightness contrast and, for $g>0$, increase the brightness contrast of the image. Minimum and maximum lightness of the image remain at their original values, while the average lightness value in the image remains at approximately the same level. We used nine versions of each scene, with gain-factor values of $-0.60,-0.45,-0.30,-0.15$, $0,0.15,0.30,0.45$, and 0.60 . 

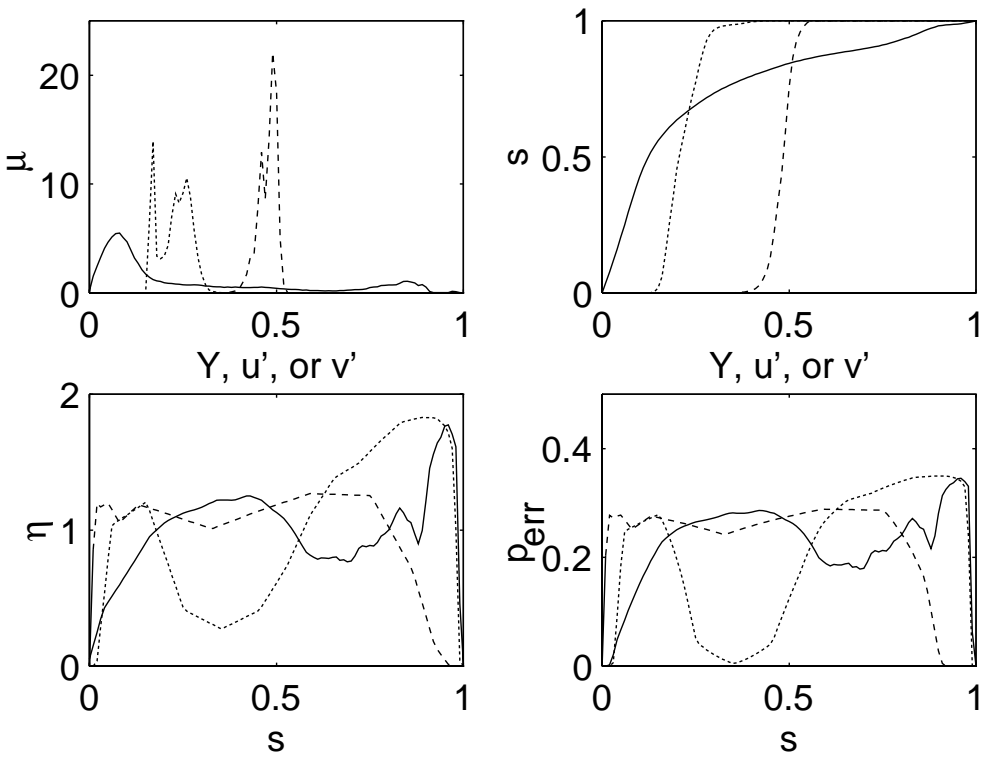

Figure 4.5: Summary of the procedure to obtain the probability of a topological error, $P_{\text {err }}$, for an image. The upper panels show the momentary distributions $\mu$ (left) and scale functions $s$ for $Y, u^{\prime}$ and $v^{\prime}$ (right) for the image of interest (note that we again used $\lambda=0.50$; these plots are identical to the ones shown in figure 4.3 in the upper right and lower right panels). The lower left panel shows the associated scale value distributions $\eta$, and the lower right panel shows $p_{e r r}$ for these distributions, assuming that $N$ is 100 and $\sigma$ is 0.01 . Integrating the areas under the curves yields $P_{e r r}$. In this particular case $P_{\text {err }}$ is $0.23,0.20$, and 0.23 for $Y, u^{\prime}$ and $v^{\prime}$, respectively. Plots for $Y$ are drawn with solid lines, those for $u^{\prime}$ with dotted lines, and those for $v^{\prime}$ with dashed lines. 


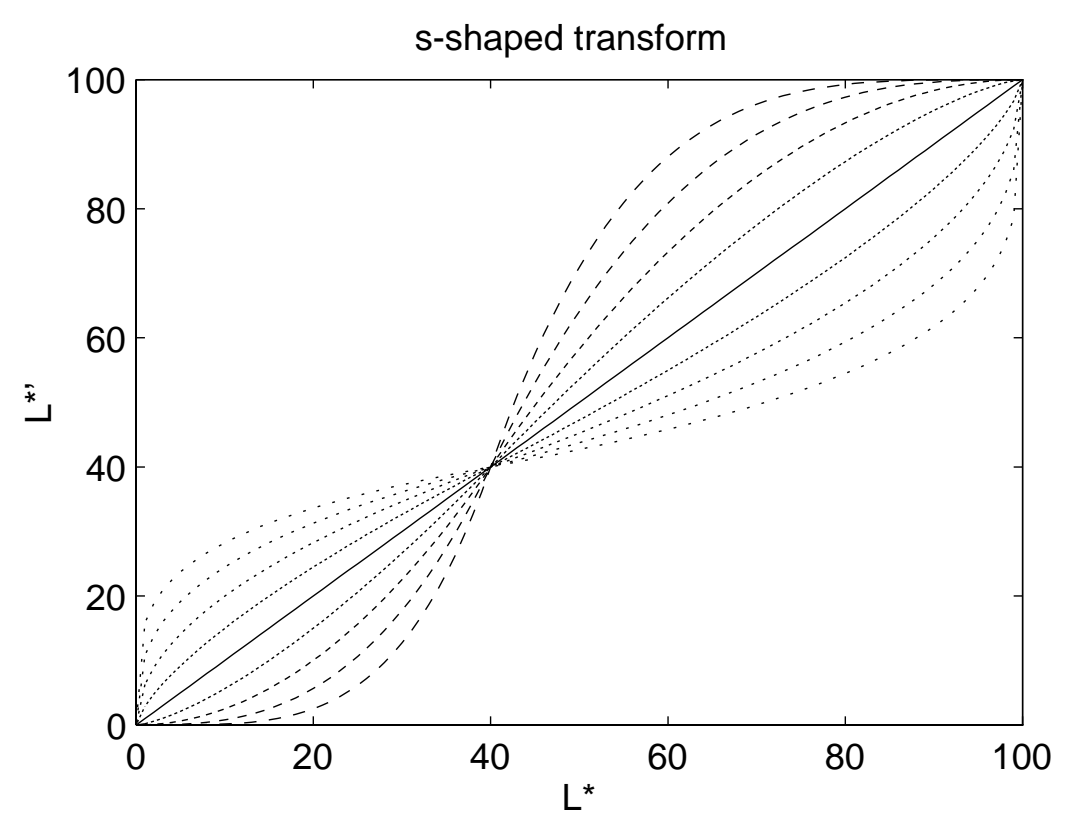

Figure 4.6: The s-shaped transformation we applied to CIE 1976 lightness $L^{*}$. On the horizontal axis the original lightness, and on the vertical axis the lightness after applying the s-shaped transformation. Curves shown are for $g=-0.60, g=-0.45, g=-0.30$, and $g=-0.15$ (dotted curves, with decreasing dot gap for increasing $g$ ), $g=0$ (solid curve), $g=0.15, g=$ $0.30, g=0.45$, and $g=0.60$ (dashed curves, with increasing dash length for increasing $g$ ). Minimum, average, and maximum values for $L^{*}$ are 0 , 40 , and 100 in this example. 

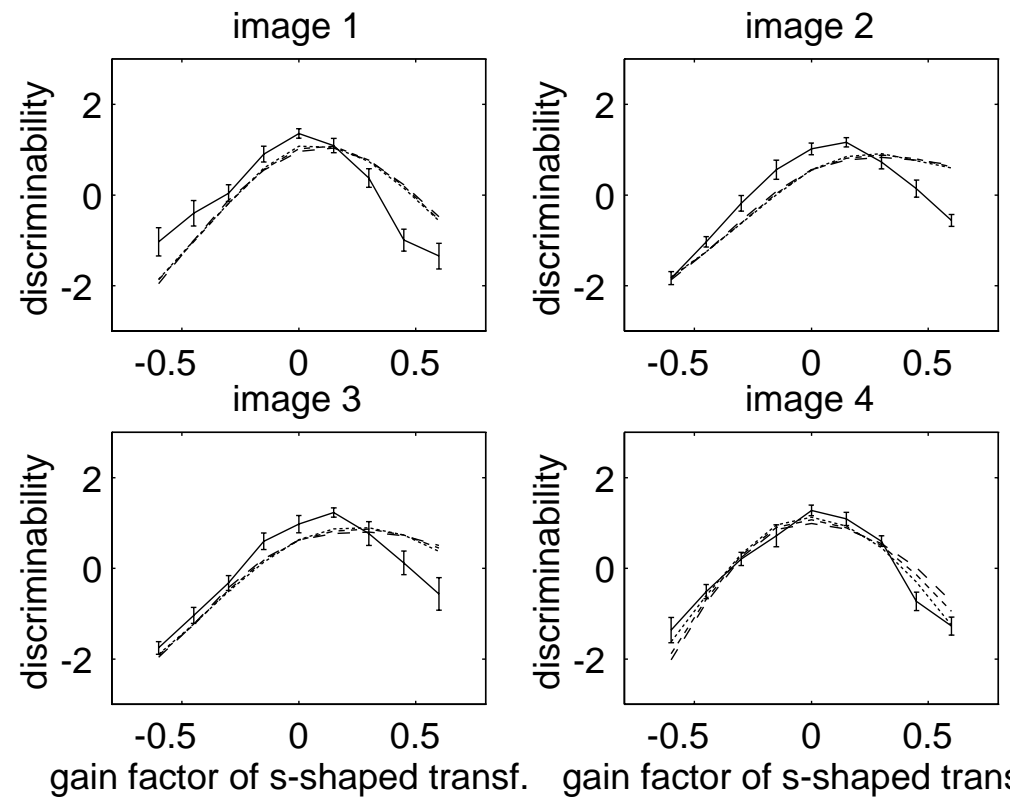

gain factor of s-shaped transf. gain factor of s-shaped transf.

Figure 4.7: Visibility of detail as judged by human subjects (solid lines, the error bars denote a distance of two standard errors in the mean) and overall discriminability $D$ (dotted lines for $\lambda=0$, short-dashed lines for $\lambda=0.25$, and long-dashed lines for $\lambda=0.5$ ) versus the gain factor of the s-shaped transformation on lightness. Results have been z-scored to facilitate a comparison.

Eight subjects were instructed to judge overall visibility of detail of the resulting images, which were shown in random order, with three replications, on a PAL-compliant CRT. For their judgments, subjects used an 11point numerical scale ranging from 0 ("bad") to 10 ("excellent"). Results, averaged over subjects and replications, are shown as the solid curves in figure 4.7. Furthermore, results produced by the algorithm are shown as dotted, short-dashed, and long-dashed curves for $\lambda=0, \lambda=0.25$, and $\lambda=0.50$, respectively. Note that to facilitate a comparison, all curves in the figure were $\mathrm{z}$-scored.

Figure 4.7 shows that subjects' judgments of visibility of detail are slightly asymmetrical around $g=0$, with visibility of detail being maximal for $g=0$ (images 1 and 4 ) to $g=0.15$ (images 2 and 3). For predictions 


\subsection{Predicting naturalness}

made by the algorithm this asymmetry is more pronounced, with maximum discriminability occurring for $g=0$ (image 4), $g=0.15$ (image 1), and $g=0.30$ (images 2 and 3). In general, predictions made by the algorithm correspond well to subjects' judgments of visibility of detail for values of $g$ below zero. For values of $g$ above zero, the algorithm tends to over-estimate discriminability compared to subjects' judgments. Subjects report that decreased visibility of detail for $g$ smaller than zero is primarily due to decreased contrast of the manipulated images. Likewise, for values of $g$ slightly larger than zero, subjects report increased visibility of detail due to increased contrast. For higher values of $g$, however, subjects report that visibility of detail is decreased due to the existence of areas in the manipulated images where detail is lost due to clipping to either black or white. Apparently, the algorithm is underestimating the impact of this effect on overall visibility of detail. Such underestimation is probably due to the fact that the algorithm is analysing luminance statistics only globally, that is, without taking into account how these statistics vary from one location in the image to another. Alternatively, subjects' attention may be drawn to areas where clipping occurs, resulting in an over-proportional influence of clipping on their judgments. Nevertheless, overall correspondence between model predictions and subjects' judgments is quite good, certainly given the assumptions and simplifications which underly the algorithm.

\subsection{Predicting naturalness}

In the introduction, we have defined naturalness in terms of the degree of match between the internal representation of an image and memory. Realising that the content of images is usually made up of objects which are more or less familiar, we may specify this further as the degree to which perceived object attributes match remembered object attributes, or memory standards. As stated in the introduction, in the algorithm we present here we will restrict ourselves to the attributes colour and brightness. To predict naturalness, we therefore need to specify: (1) how colours are internally quantified; (2) how the memory standard for a particular object's colour is constructed; and (3) how the perceived colour of an object is matched with the memory standard for that object's colour. We will address the second and third issue here, since the first issue has already been addressed in the section about metrics for brightness and colour. 


\section{Predicting the usefulness and naturalness of colour reproductions}

\subsubsection{The construction of memory standards}

When observing a particular scene, the scale function of a partially flexible metric will be determined by the distribution of the attribute strength for that scene (the momentary distribution) and by the distribution of the attribute strength for all scenes observed in the past (the eternal distribution). Observation of a particular object in this scene will therefore result in a scale value for that object which is determined by, first, the attribute strength for that object and, second, the scale function at the moment of observation. For a given attribute strength $x$ and scale function $s(x)$ the corresponding scale value $s$ is simply given by $s=s(x)$. However, the attribute strength measured for a particular object at a particular moment will usually be given by a distribution of values instead of one unique value. For example, the colour of grass in a particular scene is not uniform but instead varies from location to location. This attribute strength distribution will have its associated scale value distribution on the metric. The relation between the attribute strength distribution $\mu_{o}(x)$ and the associated scale value distribution $\eta_{o}(s)$ is simple and given by:

$$
\eta_{o}(s)=\mu_{o}(x)\left(\frac{d s(x)}{d x}\right)^{-1}
$$

where $d s(x) / d x$ is given by equation 4.1. The scale value distribution $\eta_{o}$ for an individual object will therefore depend not only on the attribute strength distribution of the object itself but also on the attribute strength distribution of the scene in which it is located.

We will assume that the memory standard for an object is constructed by the accumulation of past observations of that object, see figure 4.8. As explained above, the observation of an object will result in a distribution of scale values on the metric. Scale value distributions of past observations therefore constitute the information from which memory standards are constructed. How this accumulation is performed is unclear. Here, we will simply assume that the memory standard for a particular object is constructed by a long-term integration of the scale value distributions observed for this object. Alternatively, accumulation may also be performed by calculation and storage in memory of parameters such as mean and standard deviation of observed scale value distributions. We have chosen not to pursue this approach to avoid making assumptions about the shapes of the observed distributions. 


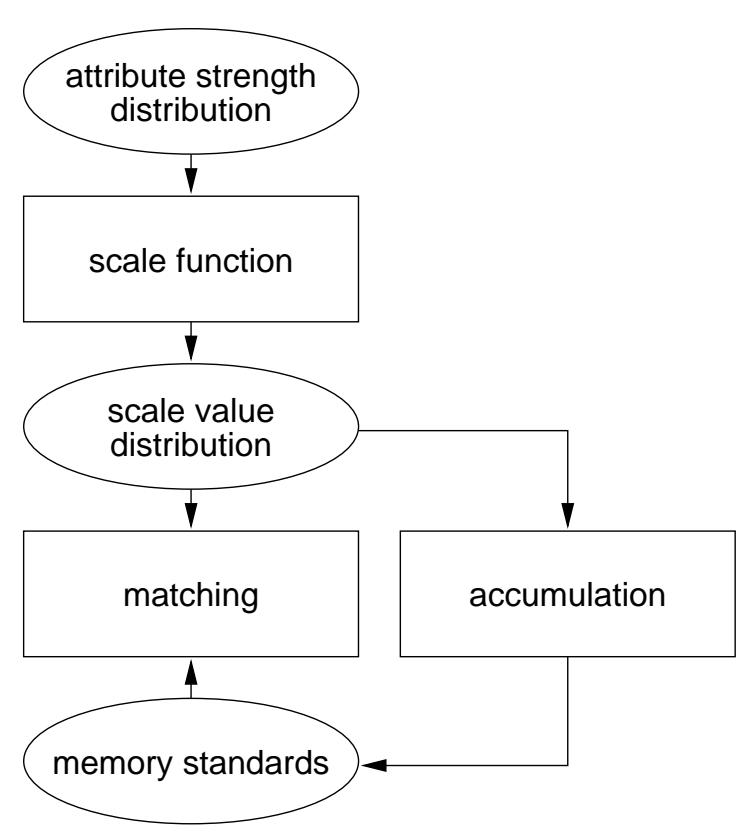

Figure 4.8: A simple model for the accumulation and matching processes. Matching is performed by means of comparing scale value distributions with standards stored in memory. The memory standards themselves are assumed to be constructed by means of accumulation of scale value distributions of past observations. 

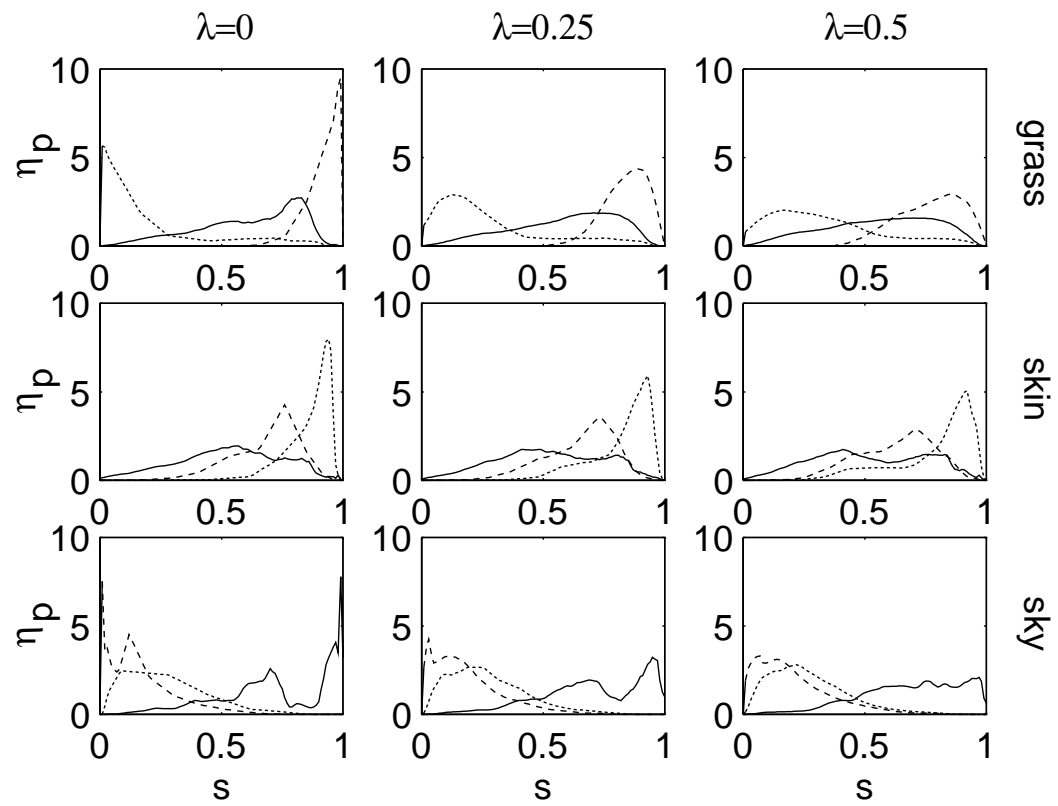

Figure 4.9: Calculated memory standards $\eta_{p}(s)$ for grass (upper row), skin (middle row), and sky (lower row) for degree of flexibility 0 (left column), 0.25 (middle column), and 0.5 (right column). Plots for $Y$ are drawn with solid lines, those for $u^{\prime}$ with dotted lines, and those for $v^{\prime}$ with dashed lines.

Figure 4.9 shows calculated memory standards for the objects grass, skin, and sky. These standards were obtained by calculating the $Y, u^{\prime}$, and $v^{\prime}$ distributions of the image areas containing grass, skin, and sky. The associated scale value distributions were then calculated using equation 4.3. Finally, the memory standards were constructed by averaging the obtained scale value distributions over the sets of images containing grass, skin, or sky. This procedure was repeated for three degrees of flexibility: $\lambda=0$, $\lambda=0.25$, and $\lambda=0.5$. 


\subsubsection{Matching perceived object colours with memory stan- dards}

The aim of the matching process is to identify an object using its observed scale value distribution. The obvious way in which this task can be accomplished is by matching the observed scale value distribution with memory standards and selecting the memory standard for which the degree of match is highest. The performance of such a mechanism may be judged by three main criteria: (1) success, that is, the overall probability of identifying correctly; (2) sensitivity, that is, the degree to which distinctions can be made in the identification of objects; and (3) robustness, that is, the degree to which the identification of an object remains stable under small variations in the observed scale value distribution as caused by, for example, noise.

There is no unique solution to this problem, and for the algorithm we present here we have made the rather arbitrary choice to express the degree of match between an observed scale value distribution and a memory standard by a normalised correlation measure:

$$
m\left(\eta_{o}(s), \eta_{p}(s)\right)=\frac{\int \eta_{o}(s) \eta_{p}(s) d s}{\sqrt{\int \eta_{o}^{2}(s) d s \int \eta_{p}^{2}(s) d s}},
$$

where $m($.$) is the degree of match, \eta_{o}$ the observed scale value distribution, and $\eta_{p}(s)$ the memory standard. The main advantage of this measure is its robustness, specifically its independence of assumptions about the shape of the distributions which are to be matched. It produces results which lie in the range zero (perfect mismatch) to one (perfect match, when $\left.\eta_{o}(s)=\eta_{p}(s)\right)$. However, since we have to deal with three dimensions, a measure of overall match must be derived from the degrees of match along the individual dimensions. The criteria which may be imposed on such a measure are: (1) overall match should increase monotonically with the degree of match in the individual dimensions; (2) overall match should be zero when there is at least one dimension for which the degree of match is zero; and (3) overall match should be one only when the degree of match is one for all individual dimensions. The obvious candidate for this measure is a simple product of the degrees of match along the individual dimensions:

$$
m=m_{\Upsilon} \cdot m_{u^{\prime}} \cdot m_{v^{\prime}},
$$




\section{Predicting the usefulness and naturalness of colour reproductions}

where $m$ is the overall degree of match, and where $m_{Y}, m_{u^{\prime}}$, and $m_{v^{\prime}}$ are the degree of match along the dimensions $Y, u^{\prime}$, and $v^{\prime}$, respectively.

To conclude, we have defined naturalness as the degree of match between perceived object attributes and memory standards. Assuming that an object has already been identified by selection of the memory standard for which the degree of match is highest, this degree of match represents the naturalness of that object. With $m$ we have a measure for predicting the naturalness of the grass, skin, and sky areas of colour reproductions of natural scenes. To predict the naturalness of the entire image, the above predictions should in principle be calculated for each individual object depicted in the image. However, since most colour manipulations applied to images are global, that is, not restricted to specific locations in the image, a weighted average of naturalness predictions for a limited set of objects depicted in the image will usually be sufficient to predict the naturalness of the entire image. Yendrikhovskij, Blommaert \& de Ridder (1999) have shown that such an approach can indeed be successfully used to predict the naturalness of an entire image from naturalness predictions for the grass, skin, and sky areas of this image.

\subsubsection{Results and discussion}

In this subsection we compare naturalness predictions produced by the above algorithm with experimental results reported by Yendrikhovskij et al. (1999). In these experiments, human subjects had to judge the naturalness of manipulated colour reproductions of natural scenes which were displayed on a CRT. To this end, the portions of the images showing grass, skin, or sky were manipulated by shifting CIE 1976 hue angle $h_{u v}$ and by scaling CIE 1976 saturation $s_{u v}$. The obtained naturalness judgments, averaged over subjects and scenes, were then plotted versus the average $u^{\prime}$ and $v^{\prime}$ coordinates of the manipulated grass, skin, or sky areas, and subsequently a two-dimensional Gaussian $f\left(u^{\prime}, v^{\prime}\right)$ was fitted to this data:

$$
\begin{aligned}
f\left(u^{\prime}, v^{\prime}\right) & \propto \exp -\frac{1}{2\left(1-\rho_{u^{\prime} v^{\prime}}^{2}\right)}\left(u_{n}^{\prime 2}-2 \rho_{u^{\prime} v^{\prime}} u_{n}^{\prime} v_{n}^{\prime}+v_{n}^{\prime 2}\right) \\
u_{n}^{\prime} & =\frac{u^{\prime}-\mu_{u^{\prime}}}{\sigma_{u^{\prime}}} \\
v_{n}^{\prime} & =\frac{v^{\prime}-\mu_{v^{\prime}}}{\sigma_{v^{\prime}}}
\end{aligned}
$$


where $\mu_{u^{\prime}}, \sigma_{u^{\prime}}, \mu_{v^{\prime}}, \sigma_{v^{\prime}}$, and $\rho_{u^{\prime} v^{\prime}}$ were the free parameters to be fitted. Finally, the authors plotted what they referred to as "one-sigma ellipses" in the $\left(u^{\prime}, v^{\prime}\right)$ plane. These ellipses, which connect locations of equal naturalness in the $\left(u^{\prime}, v^{\prime}\right)$ plane, are given by the equation:

$$
-\frac{1}{2\left(1-\rho_{u^{\prime} v^{\prime}}^{2}\right)}\left(u_{n}^{\prime 2}-2 \rho_{u^{\prime} v^{\prime}} u_{n}^{\prime} v_{n}^{\prime}+v_{n}^{\prime 2}\right)=-\frac{1}{2} .
$$

To compare the naturalness predictions as produced by the algorithm with the data of Yendrikhovskij et al., we applied the same manipulations to the grass, skin, or sky areas of twelve images which were selected from the set of 77 images $^{3}$. In particular, hue angle $h_{u v}$ was shifted by $-\frac{4}{8} \pi,-\frac{3}{8} \pi,-\frac{2}{8} \pi$, $-\frac{1}{8} \pi, 0, \frac{1}{8} \pi, \frac{2}{8} \pi, \frac{3}{8} \pi$, and $\frac{4}{8} \pi$, and saturation $s_{u v}$ was scaled by $0.41,0.51$, $0.64,0.80,1.00,1.25,1.56,1.95$, and 2.44. Naturalness of the manipulated grass, skin, or sky areas was then predicted using the algorithm, and a Gaussian fit was made for each image separately. Resulting one-sigma ellipses for these fits are shown in figure 4.10 for four images of which the grass areas were manipulated, in figure 4.11 for four images of which the skin areas were manipulated, and in figure 4.12 for four images of which the sky areas were manipulated.

In general, locations, sizes, and orientations of the one-sigma ellipses fitted to the algorithm's predictions correspond well to the one-sigma ellipses found by Yendrikhovskij et al. A degree of flexibility $\lambda=0.25$ produces best results for grass, $\lambda=0$ produces best results for skin, and $\lambda=0.50$ produces best results for sky. This seems to indicate that some degree of flexibility is needed to produce best overall results. It would nevertheless be too strong to conclude this, since the validity of such a conclusion is strongly influenced by the characteristics of the set of 77 images which were used to "train" the algorithm. This influence is difficult to estimate since there is no set of images which can be called representative for what humans observe in everyday life. Nevertheless, given the constraints imposed by having to use a limited set of images, the algorithm produces results which correspond quite well with results produced by human subjects.

\footnotetext{
${ }^{3}$ We are aware that by selecting our test images from the set of images used to construct memory standards, we are introducing some unwanted correlation between the test images and the memory standards. For the construction of one-sigma ellipses we expect this effect to be negligibly small, since the one-sigma ellipses are constructed using 81 versions (nine saturation settings times nine hue settings) of each test image. Of these 81 versions, only one version corresponds to the image which is originally used in the construction of the memory standard.
} 

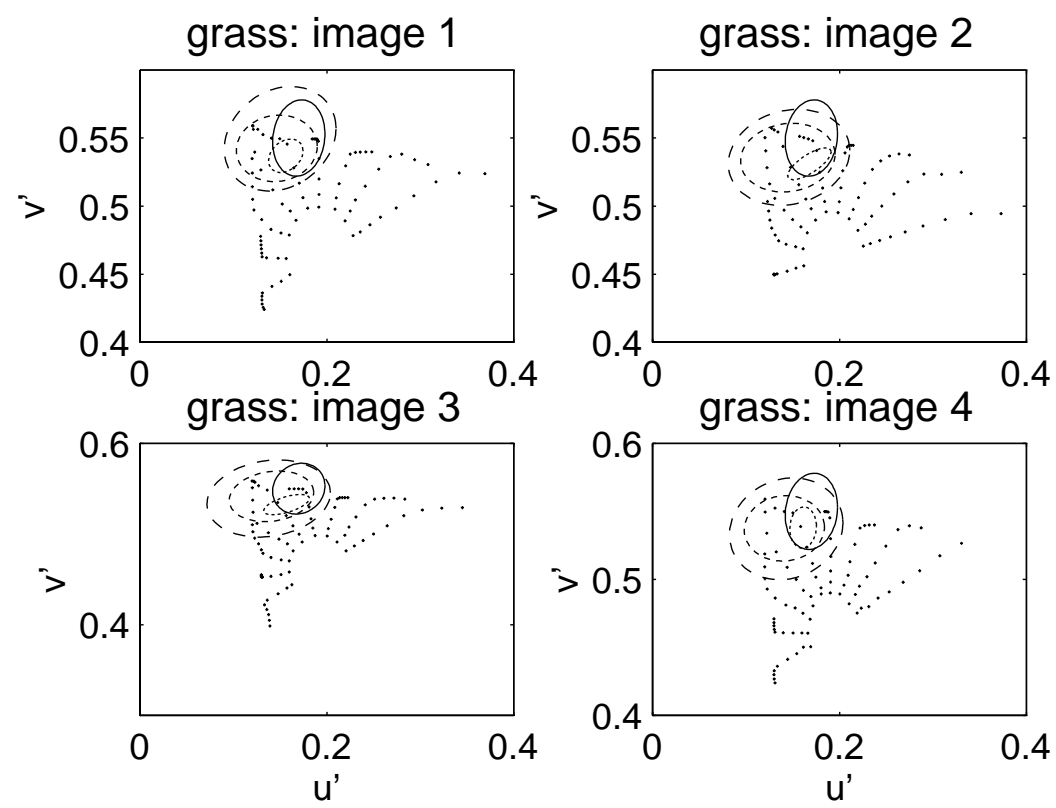

Figure 4.10: Comparison of the results of Yendrikhovskij et al. with predictions made by the algorithm for images of which the grass areas were manipulated. One-sigma ellipses for degrees of flexibility $\lambda=0, \lambda=0.25$, and $\lambda=0.50$ are shown with dotted, short-dashed, and long-dashed lines, respectively. The one-sigma ellipse found by Yendrikhovskij et al. is shown with a solid line. The dots represent the average $\left(u^{\prime}, v^{\prime}\right)$ coordinates of the grass area of the manipulated images. 

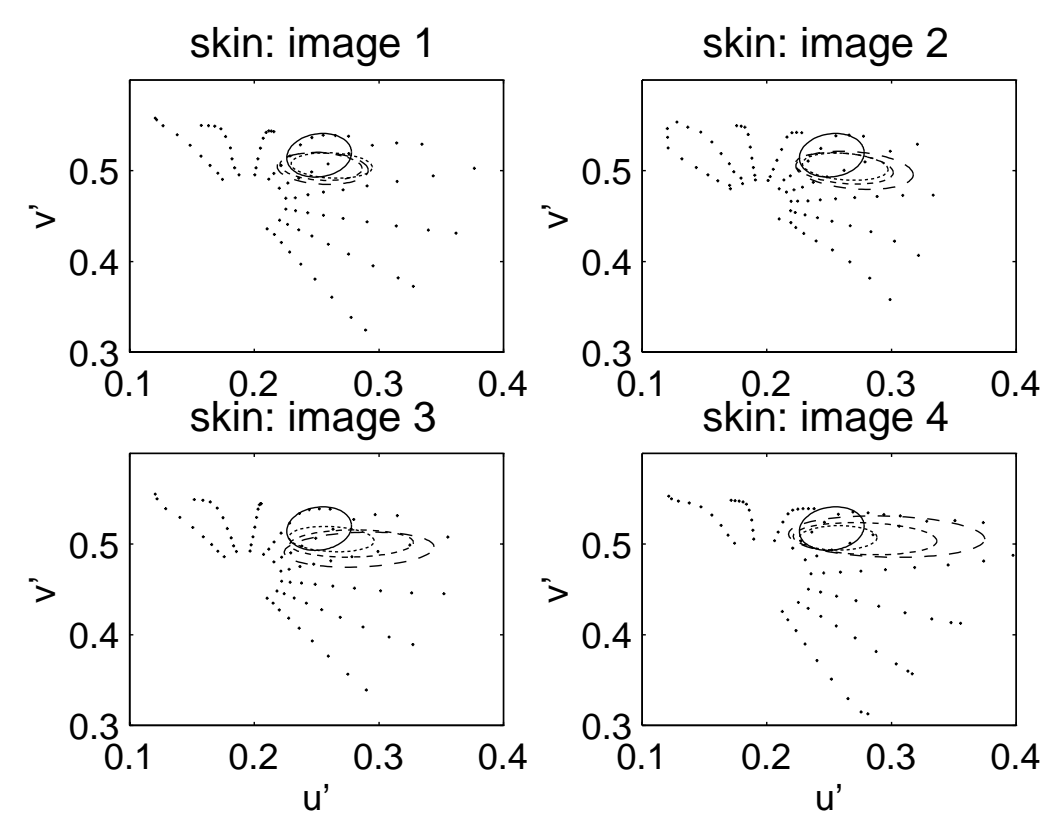

Figure 4.11: Comparison of the results of Yendrikhovskij et al. with predictions made by the algorithm for images of which the skin areas were manipulated. One-sigma ellipses for degrees of flexibility $\lambda=0, \lambda=0.25$, and $\lambda=0.50$ are shown with dotted, short-dashed, and long-dashed lines, respectively. The one-sigma ellipse found by Yendrikhovskij et al. is shown with a solid line. The dots represent the average $\left(u^{\prime}, v^{\prime}\right)$ coordinates of the skin area of the manipulated images. 

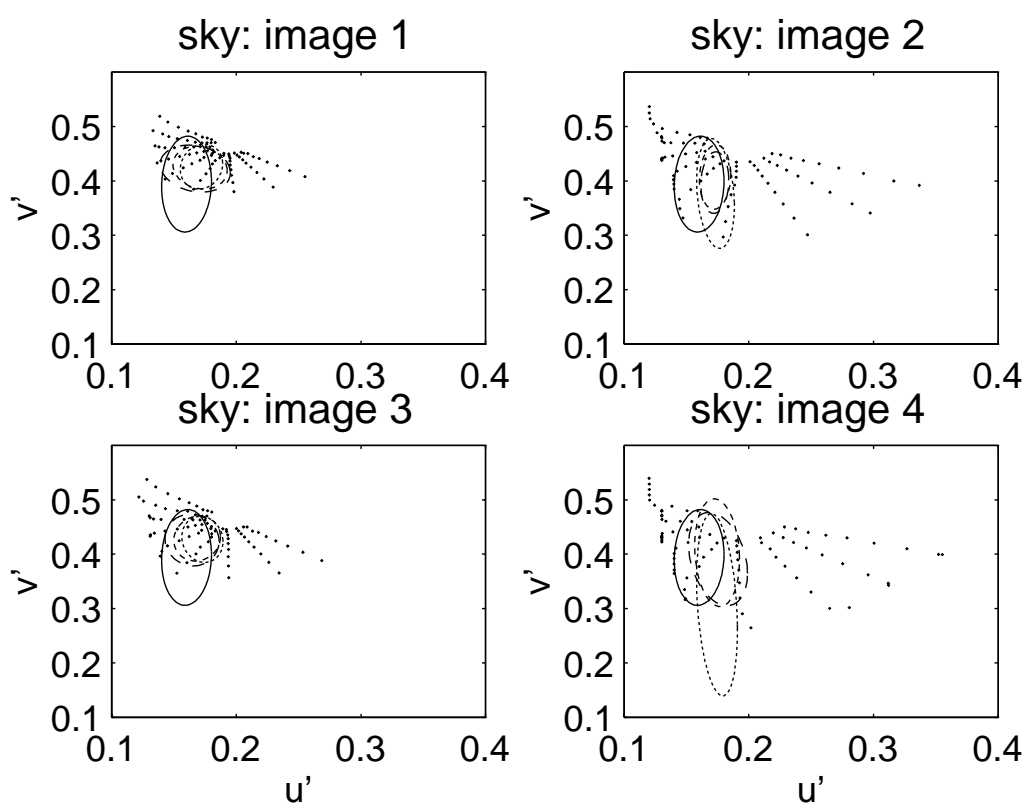

Figure 4.12: Comparison of the results of Yendrikhovskij et al. with predictions made by the algorithm for images of which the sky areas were manipulated. One-sigma ellipses for degrees of flexibility $\lambda=0, \lambda=0.25$, and $\lambda=0.50$ are shown with dotted, short-dashed, and long-dashed lines, respectively. The one-sigma ellipse found by Yendrikhovskij et al. is shown with a solid line. The dots represent the average $\left(u^{\prime}, v^{\prime}\right)$ coordinates of the sky area of the manipulated images. 


\subsection{Conclusions}

We have presented algorithms for predicting the usefulness and naturalness of colour reproductions of natural scenes, which are the two principal components determining the quality of these reproductions. The algorithm for predicting usefulness is based on the idea that usefulness is given by the overall discriminability of the items in the image using their observed attributes. The algorithm for predicting naturalness is based on the idea that naturalness is given by the degree of match between object attributes as observed in the reproduction with standards for these attributes as stored in memory. To predict usefulness and naturalness, the following stages must therefore be specified: (1) the observation of object attributes, that is, the internal quantification of these attributes; (2) the construction of memory standards from object attributes as observed in the past; and (3) the matching of observed object attributes with memory standards. For the internal quantification of object attributes we have used the concept of partially flexible metrics presented in chapter 3 , for the specification of memory standards we have assumed a simple accumulation of object attribute distributions observed in the past, and for the matching process we have assumed a simple correlation between observed object attribute distributions and memory standards. The usefulness algorithm was tested by using it to predict the discriminability of detail in manipulated black-andwhite images of natural scenes, and the naturalness algorithm was tested by using it to predict the naturalness of the grass, skin, and sky areas of manipulated colour reproductions of natural scenes. The predictions produced by the algorithms correspond quite well with experimentally obtained judgments of human subjects. 


\title{
Chapter 5
}

\section{Image quality revisited}

\begin{abstract}
We present a concept for image quality which is based on a definition of quality in terms of the degree to which something satisfies the requirements imposed on it. An answer to the question what image quality is, must therefore necessarily include answers to the questions: what are images, what are images used for, and what are the requirements which the use of images imposes on them. In this paper we therefore start by formulating answers to these questions. To this end, we distinguish two main requirements which are imposed upon images. First, the items in the image should be successfully discriminable and, second, the items in the image should be successfully identifiable. Based on the concept of (partially) flexible metrics presented in chapter 3 we then formulate algorithms for predicting discriminability, identifiability, and overall performance. To demonstrate the validity of this concept, we compare predictions made with these algorithms with experimentally obtained judgments of human subjects.
\end{abstract}

\subsection{Introduction}

Image quality is usually described in terms of the presence of visible distortions of the image such as colour shifts, blur, noise, or blockiness. Historically, the most common way to model and predict image quality therefore has been a quantification of the visibility of these distortions, for ex-

${ }^{0}$ This chapter is a slightly modified version of Janssen \& Blommaert (1999a). 
ample using models of early visual processing or using judgments of human subjects obtained in psychophysical experiments. Since this approach seems so obvious, the question of what image quality really is has traditionally been neglected, denying a better interpretation and deeper understanding of what has been measured and modelled during the past.

Our aim here is to answer the above question what image quality is, and to present a concept for image quality which is both generic and directly applicable. The concept is generic in the sense that the approach we use here can equally well be used to describe sound or speech quality. Furthermore, it is directly applicable in the sense that the description we give of image quality, and the algorithms we base upon this description, follow straightforwardly from the answer to the above question. Finally, to show the value of this concept for real applications, we show some predictions made with algorithms based on this concept together with corresponding judgments of human subjects.

\subsection{What is image quality?}

There can be no good answer to the question "what is image quality?" when the question "what is quality?" has not been answered. Perhaps the most general answer to this last question is "the degree to which something satisfies the requirements imposed on it". Though vague, this answer certainly is intuitively correct. We tend to think of things as "bad", "good", or "excellent" according to the degree to which they exhibit desired characteristics (a book; a house), or according to the degree to which they are adequate for the task we want them to perform (a car; a washing machine). So, if we wish to answer the question what image quality is, we will need to answer the questions: (1) what are images; (2) what are images used for; and (3) what are the requirements which the use of images imposes on them.

To begin with the answers to the first two questions, there can be no doubt that images are the carriers of visual information about the outside world, and that they are used as input to human visual perception. Visual perception itself is part of the three processes perception, cognition, and action, which together constitute human interaction with the environment (see figure 5.1). Images, therefore, can be regarded as input to the perception stage of interaction. If we use a rather technical view of perception, we may define perception as the stage of human interaction in which at- 


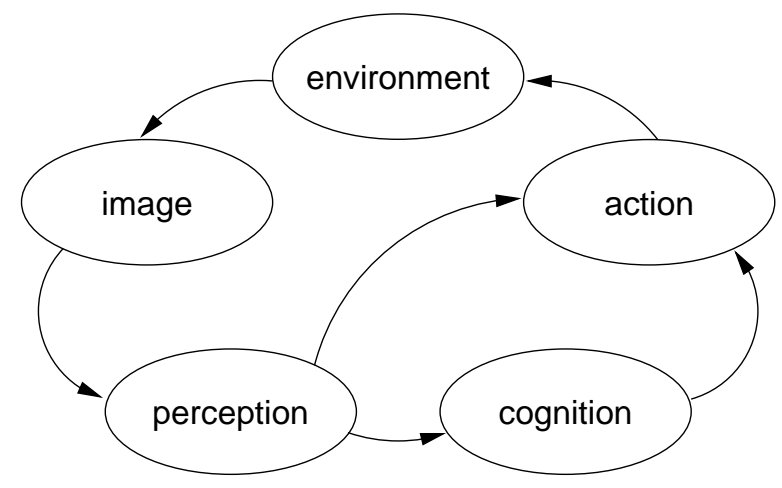

Figure 5.1: Schematic overview of the interaction process. Images are the carriers of information about the environment, and serve as input to visual perception. The result of visual processing is used as input to cognition (for tasks requiring interpretation of scene content) or as input to action (for example in navigation, where the link between perception and action is mostly direct). Since action will in general result in a changed status of the environment, the nature of the interaction process is cyclic.

tributes of items in the outside world are measured and internally quantified. The aim of this quantification is essentially two-fold. First, items in the outside world can be discriminated from one another using their internally quantified attributes. The result of this process is an essential step towards the construction of higher-level descriptions of scene geometry and object location, descriptions upon which later processes such as navigation in the scene are based. Second, items in the outside world can be identified by comparing their internally quantified attributes with quantified attributes, stored in memory, of similar items observed in the past. Identification of what is depicted in the image is an essential step in the interpretation of scene content; it determines our semantic awareness of what is in the scene.

So far, what we have found is that images are used as input to the perception stage of human interaction, and that the primary task of perception is to measure and internally quantify attributes of items in the outside world with the aim to discriminate and identify these items. These observations lead us to the answer to the third question: the requirements which the use of images, that is, their use as input to perception, imposes on them, is that, first, the items depicted in the image should be successfully discriminable, 
and, second, the items depicted in the image should be successfully identifiable. We are now able to formulate the following answer to the question what image quality is: (1) The quality of an image is the adequacy of this image as input to visual perception. (2) The adequacy of an image as input to visual perception is given by the discriminability and identifiability of the items depicted in the image. This concludes our discussion of what image quality is.

\subsection{The internal quantification of attributes}

If we wish to predict image quality, we will need to specify how discrimination and identification of items is performed, and to do this we need to specify how item attributes are internally quantified. The instrument for the quantification of attribute strength is the metric. Metrics are defined by an origin and a unit, which together constitute the scale, and a scale function, which relates physical attribute strength to the position on the scale, or scale value (Watt 1989, Watt 1991). The scale function is usually assumed to be rigid, that is, constant in time. Essentially all metrics defined by humans are of this type, the reason for this being that we wish to use these metrics to uniquely specify attribute strength in terms of scale value; 1.78 metres should be one and the same length, wherever and whenever it is measured.

As we have pointed out, perception is the stage in human interaction with the environment in which attributes of items in the outside world are measured and internally quantified, with the aim to discriminate and identify these items. The metrics which are used for this task must somehow be physiologically implemented. Any such physiological implementation, and in general any physical implementation of a metric, will inevitably result in, first, a limited scale range and, second, a limited precision with which scale values can be represented. Therefore, if we consider the limited precision with which scale values can be represented to be a source of noise, the ability to discriminate two items using their internally represented scale values will essentially be determined by the ratio of the difference of their scale values to the noise level. This, together with the limited scale range, represents an upper limit to the discriminative power of any physically implemented metric.

Watt (1991) has argued that the discriminative power of a metric can be considerably increased when the scale function of that metric is allowed to 


\subsection{The internal quantification of attributes}

be flexible instead of rigid. Flexibility allows for an adaptation of the scale function to what is currently being measured, thus sacrificing a unique specification of measured item attributes for an improved ability to discriminate these items. In chapter 3 we have extended this idea to find expressions for scale functions which are optimal with respect to discriminative power. We found that such metrics exhibit properties which resemble several well-known characteristics of human colour vision, such as adaptation, crispening, and brightness- and colour constancy. However, since flexibility of the scale function no longer allows for a unique specification of measured item attributes, the ability to compare what is observed at the moment with what has been observed in the past is reduced, resulting in a reduced ability to identify. The solution for this problem is to use partially flexible scale functions (chapter 3 ). The degree of flexibility of such scale functions can then be used to optimise the overall performance in terms of both discriminability and identifiability.

Since the aim of quantification in perception is to discriminate and identify items, and since discrimination and identification are of such importance to a successful interaction, it is to be expected that the properties of the metrics used for this quantification are optimally, or nearly optimally, chosen. We will therefore adopt the concept of partially flexible metrics here, and start by deriving expressions for metrics which are optimal with respect to either discriminability, identifiability, or a combination of these two. Based on the expressions we derive, we will then formulate expressions for discriminability and identifiability. To this end, we will regard the outside world as a simple set of items of which the values of a one-dimensional attribute are measured and quantified with the aim to discriminate and identify these items. We will further simplify the situation by assuming that the influence of external noise sources, that is, noise sources acting directly on the attribute strength, may be neglected. In the last part of this paper we will apply the obtained measures for discriminability and identifiability to predict the quality of reproduced blackand-white images of natural scenes of which the contrast has been manipulated, and compare the predictions with experimentally obtained judgments of human subjects. 


\subsection{An optimal metric for overall discriminability}

Assume that, at a certain point in time, the strength of an attribute $x$ is measured for a set of $N$ items. The momentary distribution ${ }^{1}$ of the strength of $x$ for the items in the set is given by $\mu(x, t)$, where $\mu(x, t)$ is scaled to yield unity when integrated over the range $X$ of the attribute strength:

$$
\int_{X} \mu(x, t) d x=1
$$

The measurement results are represented by scale values on an internal scale $s$. The range of the scale $s$ is given by $S$, and the scale function $s(x, t)$ which relates attribute strength $x$ to scale value $s$ is monotonic with $x$, that is, $d s(x, t) / d x \geq 0$. Furthermore, assume that the precision with which scale values can be represented is limited by the presence of Gaussian noise with mean zero and spread $\sigma$. Finally, assume that the influence of external noise on the attribute strength $x$ may be neglected.

The questions we now ask are: (1) when the items are to be discriminated using their scale values, what is the overall discriminability of the items in the set (expressed in the overall probability of a topological error) for a given scale function $s(x, t)$; and (2) what scale function $s(x, t)$ will optimise this overall discriminability (that is, minimise the overall probability of a topological error $\left.{ }^{2}\right)$ ?

\subsubsection{A measure for overall discriminability}

The scale function $s(x, t)$ maps attribute strength $x$ to scale value $s$. When the attribute strength distribution of the set of $N$ items is given by $\mu(x, t)$, then the corresponding scale value distribution $\eta(s(x, t))$ will be determined by the relation:

\footnotetext{
${ }^{1}$ We will assume that the momentary distribution $\mu(x, t)$ varies slowly with respect to the time in which adaptation-like effects occur. We assume this since we are not interested in a description of temporal aspects here. At any moment in time we will therefore regard the momentary distribution $\mu(x, t)$ as quasi-static.

${ }^{2}$ Topological errors occur when the ordering of the set of items by their scale values differs from the ordering by their attribute strengths. The occurrence of topological errors is an adequate indicator of poor discriminability, and we will therefore substitute the problem of minimising the probability of a topological error for the problem of maximising discriminability.
} 


$$
\eta(s(x, t)) d s(x, t)=\mu(x, t) d x,
$$

so that:

$$
\eta(s(x, t))=\mu(x, t)\left(\frac{d s(x, t)}{d x}\right)^{-1} .
$$

When the items are ordered by their attribute strengths, we find that the attribute strength differences between subsequent items are in close approximation given by $\Delta x=1 / N \mu(x, t)$. The corresponding scale value difference will be given by $\Delta s=1 / N \eta(s(x, t))$, with $\eta(s(x, t))$ given in equation 5.3. However, due to the presence of noise on the internal scale values, the corresponding scale value $s$ for a certain attribute strength $x$ will be given by a Normal probability density function with mean $s(x, t)$ and spread $\sigma$. For an item with attribute strength $x$ and its successor with attribute strength $x+\Delta x$ we therefore find for the scale value difference a Normal probability density function with mean $\Delta s=1 / N \eta(s(x, t))$ and spread $\sigma \sqrt{2}$. A topological error occurs when $\Delta s<0$. To find the probability of a topological error, we thus have to integrate the above probability density function from $\Delta s=-\infty$ to $\Delta s=0$ :

$$
p_{d}(x, t)=\int_{-\infty}^{0} N(\Delta s ; 1 / N \eta(s(x, t)), \sigma \sqrt{2}) d(\Delta s),
$$

which after substitution of $u=(\Delta s-1 / N \eta(s(x, t))) / \sigma \sqrt{2}$ can be written as:

$$
p_{d}(x, t)=\int_{-\infty}^{-1 / N \sigma \sqrt{2} \eta(s(x, t))} N(u ; 0,1) d u
$$

or:

$$
p_{d}(x, t)=\frac{1}{2}-\frac{1}{2} \operatorname{erf} \frac{1}{2 N \sigma \eta(s(x, t))} .
$$

The overall probability of a topological error is now given by:

$$
P_{d}(t)=\int_{X} p_{d}(x, t) \mu(x, t) d x .
$$

Substituting equations 5.6 and 5.3 in this, we obtain: 


$$
P_{d}(t)=\int_{X}\left(\frac{1}{2}-\frac{1}{2} \operatorname{erf} \frac{d s(x, t) / d x}{2 N \sigma \mu(x, t)}\right) \mu(x, t) d x
$$

\subsubsection{Optimising overall discriminability}

The question we ask here is: what scale function $s(x, t)$ will minimise the overall probability of a topological error $P_{d}(t)$ for a given attribute strength distribution $\mu(x, t)$ ? Referring to equation 5.8 , we have to solve:

$$
\min _{d s(x, t) / d x} \int_{X}\left(\frac{1}{2}-\frac{1}{2} \operatorname{erf} \frac{d s(x, t) / d x}{2 N \sigma \mu(x, t)}\right) \mu(x, t) d x .
$$

In chapter 3 we have shown that the solution to this problem is given by:

$$
\frac{d s(x, t)}{d x}=S \mu(x, t),
$$

which shows that the optimal scale function $s(x, t)$ depends on the momentary distribution of the attribute strength and therefore should be flexible. For an optimal metric, the ideal, noiseless scale value $s$ for a particular attribute strength $x$ is therefore given by:

$$
s=s(x, t)=S \int_{-\infty}^{x} \mu(u, t) d u .
$$

Substituting the above solution in equation 5.8 , the overall probability of a topological error for an optimal metric is given by:

$$
P_{d, \min }=\frac{1}{2}-\frac{1}{2} \operatorname{erf} \frac{S}{2 N \sigma},
$$

which defines the upper limit of discriminability for given values of $S$, $\sigma$, and $N$. In contrast, the lower limit of discriminability is given by the maximum value of $P_{d}(t)$, which independently of the values of $S, \sigma$ and $N$ is always given by:

$$
P_{d, \max }=\frac{1}{2}
$$


Discriminability may now be specified on a scale from zero (worst) to one (best) by using:

$$
D(t)=1-\frac{P_{d}(t)-P_{d, \text { min }}}{P_{d, \text { max }}-P_{d, \text { min }}} .
$$

\subsection{An optimal metric for overall identifiability}

We will assume that identification of an item is performed by means of selecting the item whose accumulated distribution of scale values as measured in the past is most likely to give rise to the presently measured scale value. Furthermore, we will assume that an optimal scale function as specified in equation 5.11 is used for the mapping of attribute strength $x$ to internal scale value $s$.

The questions we wish to answer here, are: (1) what do the accumulated distributions of measured scale values for the items look like; (2) in what decision rule does the above identification procedure result; (3) what is the overall identifiability at a certain point in time (expressed in the overall probability of an identification error) using the above identification procedure; and (4) what scale function will optimise this overall identifiability (that is, minimise the overall probability of an identification error)?

\subsubsection{The accumulation of scale value distributions}

We have found that, for a metric which is optimal with respect to discriminability, the scale value $s$ for a particular attribute strength $x$ at a particular point in time $t$ is given by equation 5.11 . Over time, the average scale value $\bar{s}$ measured for a particular item with attribute strength $x$ will therefore be given by:

$$
\begin{aligned}
\bar{s}=s(x) & =\frac{1}{T} \int_{T} s(x, t) d t \\
& =\frac{1}{T} \int_{T} S \int_{-\infty}^{x} \mu(u, t) d u d t \\
& =S \int_{-\infty}^{x} \frac{1}{T} \int_{T} \mu(u, t) d t d u
\end{aligned}
$$




$$
=S \int_{-\infty}^{x} \mu(u) d u,
$$

where $\mu(x)$ is the average over time of the momentary attribute strength distribution $\mu(x, t)$ :

$$
\mu(x)=\frac{1}{T} \int_{T} \mu(x, t) d t .
$$

At any particular point in time, the deviation $\Delta s(x, t)$ from the average scale value given by equation 5.15 will be given by:

$$
\begin{aligned}
\Delta s(x, t) & =s(x, t)-s(x) \\
& =S \int_{-\infty}^{x} \mu(u, t) d u-s \int_{-\infty}^{x} \mu(u) d u \\
& =S \int_{-\infty}^{x}(\mu(u, t)-\mu(u)) d u .
\end{aligned}
$$

Assuming that the above difference $\Delta s(x, t)$ may be adequately described by a Normal probability density function, the mean of this distribution will be zero and the spread $\Delta s(x)$ will be given by:

$$
\begin{aligned}
\Delta s(x) & =\sqrt{\frac{1}{T} \int_{T}(\Delta s(x, t))^{2} d t} \\
& =\sqrt{\frac{1}{T} \int_{T}\left(S \int_{-\infty}^{x}(\mu(u, t)-\mu(u)) d u\right)^{2} d t} .
\end{aligned}
$$

Note that, due to the separate and independent influence of noise on the scale values, the total spread $\hat{s}$ will be higher and given by:

$$
\hat{s}=\sqrt{(\Delta s(x))^{2}+\sigma^{2}} .
$$

The accumulated scale value distribution for an item with attribute strength $x$ may now be characterised by a mean $\bar{s}$ given by equation 5.15 , and a spread $\hat{s}$ around this mean given by equation 5.19. Equation 5.19 shows that the spread of the distributions will not be constant along the scale $s$. However, for the remainder we will assume that the spread varies slowly along $s$ and that, locally on $s$, the spread may be considered constant. 


\subsection{An optimal metric for overall identifiability}

\subsubsection{A decision rule for identification}

We have assumed that identification is performed by selecting the item whose accumulated distribution of scale values as measured in the past is most likely to give rise to the presently measured scale value. Furthermore, we have found that the accumulated scale value distribution for an item with attribute strength $x$ can be characterised by a mean and a spread given by equations 5.15 and 5.19, respectively. Since we have assumed that, locally on $s$, the spread of the scale value distributions is constant, selection of the most likely distribution reduces to the simple task of selection of the distribution whose mean lies closest to the currently measured scale value. Such a strategy results in the construction of decision bounds along the scale $s$ which lie midway between the distribution means of adjacent distributions. Our task here is to find an expression for the positions of these decision bounds.

We will assume here that a set of $N_{i}$ items, representative for the entire set of observed items, is to be identified. Specifically, we will assume that (1) the momentary attribute strength distribution of the items to be identified may at any point in time be approximated by the attribute strength distribution of the set of $N$ observed items; and (2) the attribute strength distribution of the entire set of $N_{i}$ items to be identified may be approximated by the average over time of the momentary attribute strength distribution of the set of observed items, as specified by equation 5.16 .

Having assumed this, we are now able to formulate an expression for the positions of the decision bounds for the set of $N_{i}$ items defined above. We have found that the scale value distribution mean $\bar{s}$ of an item with attribute strength $x$ is given by $s(x)$. When the attribute strength distribution of the set of $N_{i}$ items is given by $\mu(x)$, the resulting distribution $\eta(s(x))$ of the scale value distribution means will be given by:

$$
\eta(s(x)) d s(x)=\mu(x) d x
$$

so that:

$$
\eta(s(x))=\mu(x)\left(\frac{d s(x)}{d x}\right)^{-1}
$$

From equation 5.15 it follows that: 


$$
\frac{d s(x)}{d x}=S \mu(x) .
$$

For the distribution of the scale value distribution means we therefore find:

$$
\eta(s(x))=1 / S,
$$

that is, the distribution means of the $N_{i}$ items are distributed homogeneously along the scale $s$ and consequently are a distance $S / N_{i}$ apart. For an item with attribute strength $x$ this means that the decision bounds for selecting this item are given by $s(x) \pm S / 2 N_{i}$, with $s(x)$ given by equation 5.15 .

\subsubsection{A measure for overall identifiability}

We are now ready to formulate an expression for the overall identifiability at a particular point in time. The scale value observed for an item with attribute strength $x$ will, at a particular point in time, be given by a Normal probability density function with mean $s(x, t)$ given by equation 5.11 and a spread around this mean of $\sigma$. The item will be correctly identified when this scale value lies within the decision bounds which identify it as the item with attribute strength $x$, that is, when it lies within the interval $s(x) \pm$ $S / 2 N_{i}$. To find the probability of an identification error, we therefore have to integrate this probability density function over the interval $s=s(x)-$ $S / 2 N_{i}$ to $s=s(x)+S / 2 N_{i}$ and subtract the result from one:

$$
p_{i}(x, t)=1-\int_{s(x)-S / 2 N_{i}}^{s(x)+S / 2 N_{i}} N(s ; s(x, t), \sigma) d s,
$$

which after substitution of $u=(s-s(x, t)) / \sigma$ and of $\Delta s(x, t)=s(x, t)-s(x)$ can be written as:

$$
p_{i}(x, t)=1-\int_{\left(-\Delta s(x, t)-S / 2 N_{i}\right) / \sigma}^{\left(-\Delta s(x, t)+S / 2 N_{i}\right) / \sigma} N(u ; 0,1) d u,
$$

or as: 


$$
p_{i}(x, t)=1+\frac{1}{2} \operatorname{erf} \frac{\Delta s(x, t)-S / 2 N_{i}}{\sigma \sqrt{2}}-\frac{1}{2} \operatorname{erf} \frac{\Delta s(x, t)+S / 2 N_{i}}{\sigma \sqrt{2}} .
$$

The overall probability of an identification error at a particular point in time is now given by:

$$
P_{i}(t)=\int_{X} p_{i}(x, t) \mu(x, t) d x,
$$

which again assumes that the attribute strength distribution of the items to be identified may at any point in time be approximated by the attribute strength distribution of the set of $N$ items. Substituting equation 5.26, we obtain:

$$
P_{i}(t)=\int_{X}\left(1+\frac{1}{2} \operatorname{erf} \frac{\Delta s(x, t)-S / 2 N_{i}}{\sigma \sqrt{2}}-\frac{1}{2} \operatorname{erf} \frac{\Delta s(x, t)+S / 2 N_{i}}{\sigma \sqrt{2}}\right) \mu(x, t) d x,
$$

where $\Delta s(x, t)$ is given by equation 5.17 .

\subsubsection{Optimising overall identifiability}

The problem we wish to solve here, is: what scale function $s(x, t)$ will minimise the overall probability of an identification error $P_{i}(t)$ for given attribute strength distributions $\mu(x, t)$ and $\mu(x)$ ? Referring to equation 5.28, we have to solve:

$$
\min _{\Delta s(x, t)} \int_{X}\left(1+\frac{1}{2} \operatorname{erf} \frac{\Delta s(x, t)-S / 2 N_{i}}{\sigma \sqrt{2}}-\frac{1}{2} \operatorname{erf} \frac{\Delta s(x, t)+S / 2 N_{i}}{\sigma \sqrt{2}}\right) \mu(x, t) d x,
$$

and then derive $s(x, t)$ from $\Delta s(x, t)$ and $s(x)$ using equations 5.17 and 5.15 . The solution to this problem is easily found: if we wish to minimise equation 5.28 , we will have to find a $\Delta s(x, t)$ such that $p_{i}(x, t)$ is minimised for all $x$. Referring to equation 5.26 , we are looking for a $\Delta s(x, t)$ such that:

$$
\operatorname{erf} \frac{\Delta s(x, t)+S / 2 N_{i}}{\sigma \sqrt{2}}-\operatorname{erf} \frac{\Delta s(x, t)-S / 2 N_{i}}{\sigma \sqrt{2}}
$$


is maximised. This equation represents the area under the curve of a Normal probability density function with mean zero and spread $\sigma$ over the interval $\Delta s(x, t)-S / 2 N_{i}$ to $\Delta s(x, t)+S / 2 N_{i}$. Evidently, this area is maximised when the interval lies symmetrically around the mean of the distribution, that is, when $\Delta s(x, t)=0$. From equation 5.17 it follows that for $\Delta s(x, t)$ to be zero, $s(x, t)$ should be equal to $s(x)$, with $s(x)$ given by equation 5.15 . For an optimal metric, we therefore find here:

$$
s(x, t)=s(x)=s \int_{-\infty}^{x} \mu(u) d u,
$$

with $\mu(u)$ the average over time of the momentary frequency distribution $\mu(x, t)$. Equation 5.31 shows that the scale function $s(x, t)$ should in this case be rigid. Substituting the solution we find here in equation 5.28, the overall probability of an identification error for an optimal metric is given by:

$$
P_{i, \min }=1-\operatorname{erf} \frac{S}{2 \sqrt{2} N_{i} \sigma},
$$

which defines the upper limit of identifiability for given $S, \sigma$, and $N_{i}$. The lower limit of identifiability will be given by the maximum value of $P_{i}(t)$, which independently of $S$ and $\sigma$ and for $N_{i}>1$ is given by:

$$
P_{i, \max }=1 .
$$

Identifiability may now be specified on a scale from zero (worst) to one (best) by using:

$$
I(t)=1-\frac{P_{i}(t)-P_{i, \min }}{P_{i, \max }-P_{i, \min }} .
$$

\subsection{Estimating the number of discriminable and identifiable items}

To estimate the number of discriminable items, we return to equation 5.12. When we rewrite this equation to: 


$$
N=\frac{S}{2 \sigma \operatorname{erf}^{-1}\left(1-2 P_{d}\right)}
$$

this equation allows us to estimate the maximum number of discriminable items using an optimal metric for a given allowable error probability $P_{d}$ of, say, $25 \%$, and a given internal dynamic range $S / \sigma$. A plot of the maximum number of discriminable items versus the dynamic range $S / \sigma$ is shown as the solid line in the left panel of figure 5.2.

In a similar way, we may rewrite equation 5.32 to:

$$
N_{i}=\frac{S}{2 \sqrt{2} \sigma \operatorname{erf}^{-1}\left(1-P_{i}\right)} .
$$

Here, too, the maximum number of identifiable items using an optimal metric may be estimated for a given allowable error probability $P_{i}$ and a given dynamic range $S / \sigma$. The solid line in the right panel of figure 5.2 shows a plot of the maximum number of identifiable items versus the dynamic range $S / \sigma$ obtained this way for $P_{i}=25 \%$.

Figure 5.2 shows that, for an allowable error probability of $25 \%$, the number of discriminable items $N$ using a metric which is optimal with respect to discriminability is about the same as the dynamic range $S / \sigma$. The number of identifiable items $N_{i}$ using a metric which is optimal with respect to identifiability is, however, a factor 2.4 smaller than the dynamic range.

\subsection{Partial flexibility}

We have found expressions for overall discriminability (equation 5.8) and overall identifiability (equation 5.28). Furthermore, we have found that optimising overall discriminability requires that the scale function be flexible (equation 5.11), and that optimising overall identifiability requires that it be rigid (equation 5.31). What we wish to do here, is to find a partially flexible scale function which is chosen such that the total performance (in terms of both discriminability and identifiability) is optimised. This problem can be solved using regularisation, see, for example, Poggio, Torre \& Koch (1990). If we express the total performance $T(t)$ in terms of the discriminability $D(t)$ and identifiability $I(t)$, total performance can be optimised by solving: 

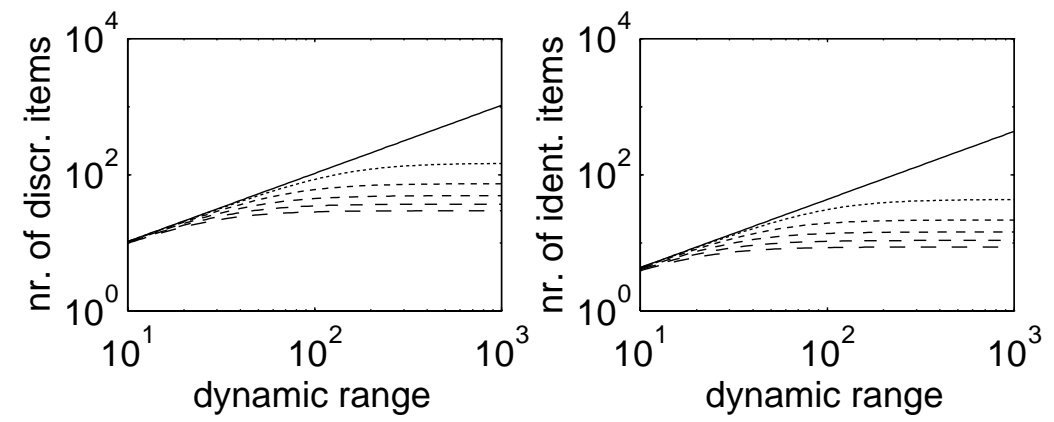

Figure 5.2: The number of discriminable items (left panel) and identifiable items (right panel) versus the dynamic range $S / \sigma$ of the scale, for a maximum allowable error probability of $25 \%$. The solid lines represent optimum performance, that is, a completely flexible metric for discriminability and a completely rigid metric for identifiability. The dashed lines show the influence of decreased flexibility for the number of discriminable items, and the influence of decreased rigidity for the number of identifiable items. See section 5.7.3 for how the dashed curves have been obtained.

$$
\min _{s(x, t)} T(t)=\epsilon D(t)+(1-\epsilon) I(t),
$$

where $\epsilon$ expresses the relative importances of discriminability and identifiability for the overall performance.

Here, we will assume that the general solution to this problem belongs to the class of scale functions:

$$
s(x, t)=\lambda S \int_{-\infty}^{x} \mu(u, t) d u+(1-\lambda) S \int_{-\infty}^{x} \mu(u) d u,
$$

where the optimal value of the parameter $\lambda$ will depend on the choice made for $\epsilon$. The parameter $\lambda$ can be interpreted as the degree of flexibility of the scale function, since for $\lambda=0$ we obtain a completely rigid scale function which is optimal with respect to identifiability, while for $\lambda=1$ we obtain a completely flexible scale function which is optimal with respect to discriminability. Note that the average over time of a partially flexible metric as specified in equation 5.38 is still given by: 


$$
\begin{aligned}
\bar{s} & =\frac{1}{T} \int_{T} s(x, t) d t \\
& =\frac{1}{T} \int_{T}\left(\lambda S \int_{-\infty}^{x} \mu(u, t) d u+(1-\lambda) S \int_{-\infty}^{x} \mu(u) d u\right) d t \\
& =\lambda S \int_{-\infty}^{x} \frac{1}{T} \int_{T} \mu(u, t) d t d u+(1-\lambda) S \int_{-\infty}^{x} \frac{1}{T} \int_{T} \mu(u) d t d u \\
& =\lambda S \int_{-\infty}^{x} \mu(u) d u+(1-\lambda) S \int_{-\infty}^{x} \mu(u) d u \\
& =S \int_{-\infty}^{x} \mu(u) d u \\
& =S(x) .
\end{aligned}
$$

Therefore, the results derived for the accumulation of scale value distributions and the decision rule based upon this accumulation are still valid.

In the remainder we will derive expressions for discriminability $D(t, \lambda)$ and identifiability $I(t, \lambda)$, given a certain degree of flexibility $\lambda$. The performance optimisation problem as formulated above now becomes:

$$
\min _{\lambda} \epsilon D(t, \lambda)+(1-\epsilon) I(t, \lambda)
$$

which can be solved by taking the derivative with respect to $\lambda$ and setting the result to zero:

$$
\frac{\partial}{\partial \lambda}(\epsilon D(t, \lambda)+(1-\epsilon) I(t, \lambda))=0
$$

\subsubsection{Discriminability for partial flexibility}

When substituting equation 5.38 into equation 5.8, we obtain for the overall probability of a topological error:

$$
P_{d}(t, \lambda)=\int_{X}\left(\frac{1}{2}-\frac{1}{2} \operatorname{erf} \frac{\lambda S \mu(x, t)+(1-\lambda) S \mu(x)}{2 N \sigma \mu(x, t)}\right) \mu(x, t) d x
$$

Discriminability $D(t, \lambda)$ can be derived from this by using equation 5.14 . 


\subsubsection{Identifiability for partial flexibility}

For the overall probability of an identification error we have already found equation 5.28:

$$
P_{i}(t, \lambda)=\int_{X}\left(1+\frac{1}{2} \operatorname{erf} \frac{\Delta s(x, t)-S / 2 N_{i}}{\sigma \sqrt{2}}-\frac{1}{2} \operatorname{erf} \frac{\Delta s(x, t)+S / 2 N_{i}}{\sigma \sqrt{2}}\right) \mu(x, t) d x .
$$

For a partially flexible metric as specified by equation $5.38, \Delta s(x, t)$ will be given by:

$$
\Delta s(x, t)=\lambda S \int_{-\infty}^{x}(\mu(u, t)-\mu(u)) d u .
$$

Identifiability $I(t, \lambda)$ can be derived from this by using equation 5.34 .

\subsubsection{Re-estimating the number of discriminable and identi- fiable items}

Our aim here is to re-estimate the number of discriminable and identifiable items, this time for a partially flexible metric as specified by equation 5.38. We will first regard the number of discriminable items. For items having a small difference $\delta x$ in their attribute strengths, the corresponding scale value difference $\delta s$ is given by $\delta s=(d s(x, t) / d x) \delta x$, where $d s(x, t) / d x$ for a flexible metric is given by $d s(x, t) / d x=\lambda S \mu(x, t)+(1-\lambda) S \mu(x)$. Furthermore, when the items are ordered by attribute strength, the attribute strength difference between subsequent items is in good approximation given by $\delta x(x, t)=1 / N \mu(x, t)$. For the corresponding scale value difference $\delta s(x, t)$ we therefore find:

$$
\delta s(x, t)=\frac{1}{N \mu(x, t)}(\lambda S \mu(x, t)+(1-\lambda) S \mu(x)) .
$$

Defining $\Delta \mu(x, t)=\mu(x, t)-\mu(x)$ we may rewrite this to:

$$
\delta s(x, t)=\frac{1}{N \mu(x, t)}(\lambda S \mu(x, t)+(1-\lambda) S(\mu(x, t)-\Delta \mu(x, t))) .
$$




$$
\begin{aligned}
& =\frac{S}{N}-\frac{S}{N}(1-\lambda) \frac{\Delta \mu(x, t)}{\mu(x, t)} \\
& =\overline{\delta s(x, t)}+\delta s(x, t) .
\end{aligned}
$$

The second term of this equation may be regarded as a source of variability on the average scale value difference $\overline{\delta s(x, t)}=S / N$, which is proportional to the degree of rigidity $(1-\lambda)$. Since this variability is primarily caused by the term $\Delta \mu(x, t) / \mu(x, t)$, we may not expect this variability to be Normally distributed. If we nevertheless assume a Normal probability density function, the mean of this distribution will be zero and the spread given by:

$$
\widehat{\delta s}=\sqrt{\frac{1}{X} \int_{X} \frac{1}{T} \int_{T}\left(\delta \widehat{s(x, t))^{2} d t d x} .\right.}
$$

For the scale value differences we have already found that the variability as caused by internal noise is given by a Normal probability density function with a spread $\sigma \sqrt{2}$. When we include the above variability as caused by rigidity, and when we assume that the approximation by a Normal probability density function is more or less valid, we find a total equivalent variability which is given by a spread of $\sigma^{\prime}=\sqrt{(\sigma \sqrt{2})^{2}+\widehat{\delta s}^{2}}$. Substituting $\sigma^{\prime}$ for $\sigma \sqrt{2}$ in equation 5.35 now allows for an estimation of the number of discriminable items for a partially flexible metric. Resulting plots for $\widehat{\delta s}=\sigma, 2 \sigma, \ldots, 5 \sigma$ are shown as the dashed lines in the left panel of figure 5.2. The figure indeed shows that the number of discriminable items is reduced when the metric is less than completely flexible. However, since $\widehat{\delta s}$ is effectively determined by the product of rigidity $(1-\lambda)$ and the difference between momentary and average distribution $\Delta \mu(x, t)$, the reduced number of discriminable items is the result of a combined effect, and reduced flexibility will therefore only result in a reduced number of discriminable items when $\mu(x, t)$ is not equal to $\mu(x)$.

Related to this, it is interesting to notice that if we regard the number of discriminable steps in attribute strength $x$, as measured in terms of JND's (justnoticeable differences), we find that this number should for rigid metrics be essentially equal to the dynamic range $S / \sigma$. For a (partially) flexible metric this no longer holds, and the dynamic range $S / \sigma$ becomes the lower limit for the number of discriminable steps. The explanation for this is as follows. For a small step $\Delta x$ in attribute strength, we find a corresponding scale value difference $\Delta s=(d s(x) / d x) \Delta x$. Any scale value difference $\Delta s$ 
which exceeds the noise level $\sigma$ on the scale effectively corresponds to a discriminable step. Therefore, $\Delta x=\sigma /(d s(x) / d x)$ defines the size of a discriminable step. Due to adaptation of the (partially) flexible metric to the momentary frequency distribution of the attribute strength, $d s(x) / d x$ will always be relatively high for the value of $x$ currently used as the reference for $\Delta x$. The resulting discriminable step size $\Delta x$ will, for a (partially) flexible metric, therefore be relatively small, and the total number of discriminable steps correspondingly high. The conclusion which may be drawn from this is that measurement of the dynamic range of a certain metric in terms of the number of JND's will result in an over-estimation of the dynamic range when the metric under consideration is (partially) flexible.

We now turn to estimating the number of identifiable items. The use of a partially flexible metric as given in equation 5.38 will result in a mapping from attribute strength $x$ to scale value $s$ given by:

$$
s=s(x, t)=s(x)+\Delta s(x, t),
$$

with $\Delta s(x, t)$ given by equation 5.44 . If we assume that $\Delta s(x, t)$ may be approximated by a random variable which is Normally distributed, the spread of the Normal probability density function will be given by:

$$
\hat{s}=\sqrt{\frac{1}{X} \int_{X} \frac{1}{T} \int_{T}(\Delta s(x, t))^{2} d t d x .}
$$

Using this approximation, we have found a source of variability which adds to the internal noise on the scale values and which is proportional to the degree of flexibility $\lambda$. The total equivalent noise on the scale values is therefore given by a Normal probability density function with mean zero and spread $\sigma^{\prime}$ given by:

$$
\sigma^{\prime}=\sqrt{\sigma^{2}+\hat{s}^{2}} .
$$

Substituting $\sigma^{\prime}$ for $\sigma$ in equation 5.36, we may now re-estimate the number of identifiable items when a partially flexible metric is used. Resulting plots for $\hat{s}=\sigma, 2 \sigma, \ldots, 5 \sigma$ are shown as the dashed lines in the right panel of figure 5.2.

The figure shows a profound effect of flexibility: the number of items which can be identified with a maximum allowable error probability of, in this case, $25 \%$, is no longer a fixed factor 2.4 smaller than the dynamic 
range $S / \sigma$, and rises asymptotically to a value which is essentially determined by the variability $\hat{s}$ caused by the flexibility of the metric. This result is in good correspondence with a well-known experimentally established fact: when subjects are asked to identify stimuli which vary in one-dimensional attributes like loudness, brightness, or length, they are usually unable to successfully identify the stimuli with more than the famous "seven plus or minus two" stimulus categories (Miller 1956), even though the number of discriminable steps in these attributes is in the order of one hundred.

Within the framework of (partially) flexible metrics we have found a tentative explanation for this effect: for a rigid metric we have estimated that the number of identifiable items should be about 2.4 times smaller than the dynamic range $S / \sigma$, whereas the number of discriminable steps should be essentially equal to the dynamic range. Due to the flexibility of the metric, the additional variability $\hat{s}$ in the mapping from attribute strength $x$ to scale value $s$ essentially becomes the limiting factor for the number of identifiable items, whereas the dynamic range $S / \sigma$ now becomes the lower limit for the number of discriminable steps (refer to what has been concluded earlier about the number of JND's for partially flexible metrics). We therefore expect that the ratio of the number of discriminable steps to the number of identifiable items will be around 2.4 for those attributes for which the metric is rigid, with values exceeding 2.4 for those attributes whose internal metrics are increasingly more flexible.

\subsubsection{Optimising the degree of flexibility}

Our aim here is to give an example of the solution to the flexibility optimisation problem (equation 5.41) for a simplified situation. To this end, we have assumed that the average distribution $\mu(x)$ is uniform between $x=0$ and $x=1$ and the momentary distribution $\mu(x, t)$ is Normal between $x=0$ and $x=1$ with mean 0.5 and spread 0.15 . Furthermore, we have assumed that the scale range $S$ is one, and we have assumed a dynamic range $S / \sigma$ of one hundred. For the noise level $\sigma$ these choices result in a value of 0.01 . Last, we have assumed that the number of items to be discriminated, $N$, is one hundred (the maximum number of discriminable items for the chosen dynamic range of one hundred and for a maximum allowable error probability of $25 \%$ ), and that the number of items to be identified, $N_{i}$, is ten (a number which for a partially flexible metric, a dynamic range of one hundred, and a maximum allowable error probability of $25 \%$ is close 


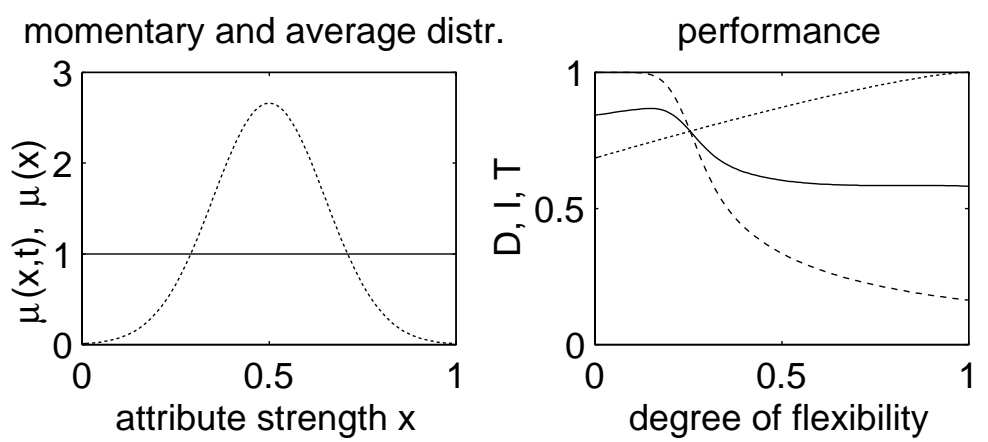

Figure 5.3: The influence of the degree of flexibility on discriminability $D$, identifiability $I$, and total performance $T$ for a simplified case. The left panel shows the assumed momentary distribution $\mu(x, t)$ (dotted line) and average distribution $\mu(x)$ (solid line). The right panel shows calculated discriminability (dotted line), identifiability (dashed line) and total performance (solid line) versus the degree of flexibility. For this particular case, total performance is optimised for a degree of flexibility of 0.15 . See text for further details about the assumed values for parameters such as dynamic range.

to the maximum number of identifiable items). Figure 5.3 shows a plot of discriminability $D$ (dotted line) and identifiability $I$ (dashed line) versus the degree of flexibility $\lambda$. The solid line represents total performance $T$ when the value of $\epsilon$ in equation 5.41 is set to 0.5 .

The plot indeed shows that discriminability rises monotonically with the degree of flexibility. It also shows that, for a degree of flexibility below approximately 0.20 , identifiability is relatively unaffected by increasing flexibility. However, for degrees of flexibility of about 0.25 , identifiability decreases dramatically with increasing flexibility. Figure 5.3 shows that improved discriminability compensates for decreased identifiability up to a degree of flexibility of 0.15 , where total performance $T$ reaches its maximum of approximately 0.87 . Therefore, for this specific case a relatively small degree of flexibility of 0.15 optimises overall performance.

To conclude, the particular degree of flexibility which optimises overall performance will in general depend on the relative importances of discriminability and identifiability, with increasing importance of discriminability resulting in a higher degree of flexibility and, conversely, increas- 


\subsection{Application: black-and-white images of natural scenes}

ing importance of identifiability resulting in a lower degree of flexibility. Quantification of outside world attributes is an early stage in perception, and we therefore expect flexible metrics to be a property of the earliest stages in perception. Having observed this, it is unlikely that the degree of flexibility can be changed from one moment to the next according to what is required by the task at hand. Instead, it is more likely that the optimal choice for flexibility will be given by the successfulness of the interaction process during a longer period in time, for example in terms of the number and severity of the errors made during the interaction.

An important parameter determining how discriminability and identifiability will vary for a given degree of flexibility is the statistical behaviour of the momentary distribution in time. Luminance is a particularly interesting case in this respect. A typical range of luminance is about three orders of magnitude at any particular moment, however the total range of luminance is estimated to be about ten orders of magnitude (McCann 1988). For a rigid metric this entire range is somehow to be mapped onto the internal scale, therefore even for a strongly compressive scale function, such as a logarithmic scale function, only about thirty percent of the scale would really be used at any particular point in time. For a flexible metric this situation is dramatically improved, with the lowest luminance in the scene mapped onto the lower end of the scale, and the highest luminance in the scale mapped onto the upper end of the scale. In this situation, scale values will roughly correspond to relative luminances, and therefore to surface reflection coefficients. Since surface reflection can be regarded as an invariant object property, the use of a flexible metric in this particular case actually helps to identify items using their measured luminances.

\subsection{Application: black-and-white images of natu- ral scenes}

Our aim in this section is to compare discriminability, identifiability, and total performance predictions for four black-and-white images of natural scenes with experimentally obtained judgments of sharpness, visibility of detail, naturalness, and quality. Judgments of sharpness and visibility of detail were chosen since these attributes are in different ways related to discriminability. Judgments of naturalness, which in the experiment was defined as the degree to which the image is realistically reproduced, were chosen since this attribute is indirectly related to identifiability; the less re- 
alistically an image is reproduced, the less well the image can be matched with what is in memory, and therefore the less identifiable the items in the image will be. The aim of this comparison will not be to analyse the accuracy with which the predictions correspond to experimental results. Instead, the aim will be to check whether several non-trivial tendencies in the obtained experimental data, most notably the differences between naturalness judgments and quality judgments reported earlier in chapter 2, can be adequately predicted.

We selected our images from a set of 77 digitised colour images of natural scenes recorded on two Kodak Photo CD's. We produced black-and-white versions of these images by transforming the images from the RGB system space to the CIELUV colour space (Hunt 1992) and setting the chromaticity coordinates $u^{*}$ and $v^{*}$ to zero. Finally, we manipulated the brightness contrast of the obtained black-and-white images by applying an s-shaped transformation on lightness $L^{*}$ :

$$
\begin{aligned}
L^{*^{\prime}}= & \left(\frac{L^{*}-L_{\min }^{*}}{L_{\text {ave }}^{*}-L_{\min }^{*}}\right)^{\gamma}\left(L_{\text {ave }}^{*}-L_{\min }^{*}\right)+L_{\min }^{*} \\
& \text { for } L_{\min }^{*} \leq L^{*} \leq L_{\text {ave }}^{*} \\
L^{*^{\prime}}= & \left(\frac{L_{\max }^{*}-L^{*}}{L_{\max }^{*}-L_{\text {ave }}^{*}}\right)^{\gamma}\left(L_{\text {ave }}^{*}-L_{\max }^{*}\right)+L_{\max }^{*} \\
& \quad \text { or } L_{\text {ave }}^{*}<L^{*} \leq L_{\max }^{*},
\end{aligned}
$$

where $L^{*}$ represents the original lightness value, $L^{*^{\prime}}$ the new value, and where $L_{\text {min }}^{*} L_{\text {max }}^{*}$, and $L_{a v e}^{*}$ represent the minimum, maximum, and average lightness in the original image, respectively. The parameter $\gamma$ in equation 5.51 is specified in terms of a gain-factor $g$ as:

$$
\gamma=10^{g} .
$$

Applying the above s-shaped transformation will decrease the brightness contrast for negative values of $g$, and increase the brightness contrast of the image for positive values of $g$, while the average lightness of the image remains at approximately the original level. Nine versions of each scene were used, with gain-factor values of $-0.60,-0.45,-0.30,-0.15,0,0.15$, $0.30,0.45$, and 0.60 . Subjects, eight in number, were in separate sessions instructed to judge sharpness, visibility of detail, naturalness, and quality of the resulting images. The images were shown in random order and 


\begin{tabular}{|c|c|c|c|c|c||c|c|c|c|c|c|}
\hline \multicolumn{9}{|c||}{$P_{i}=25 \%$} & \multicolumn{6}{c|}{$P_{i}=5 \%$} \\
\hline \hline$\lambda$ & 0.00 & 0.25 & 0.50 & 0.75 & 1.00 & $\lambda$ & 0.00 & 0.25 & 0.50 & 0.75 & 1.00 \\
\hline$N_{i}$ & 43.5 & 12.9 & 6.7 & 4.5 & 3.4 & $N_{i}$ & 25.5 & 7.6 & 3.9 & 2.6 & 2.0 \\
\hline
\end{tabular}

Table 5.1: Calculated number of identifiable items for the luminance distributions of the set of 77 images, assuming $S=1.00$ and $\sigma=0.01$ (dynamic range $S / \sigma=100$ ). The number of identifiable items was calculated for degrees of flexibility $\lambda$ of $0.00,0.25,0.50,0.75$, and 1.00 , and for a maximum allowable error probability $P_{i}$ of $25 \%$ and $5 \%$, For the predictions in this section we have chosen $P_{i}=25 \%$ and $\lambda=0.25$, hence $N_{i} \approx 13$.

with three replications on a PAL-compliant CRT (cathode ray tube). Subjects were instructed to use an 11-point numerical scale ranging from 0 ("bad") to 10 ("excellent") for their judgments. The resulting judgments were z-scored and averaged over subjects and replications.

For our predictions we assumed the following. First, for the momentary distribution $\mu(x, t)$ we used the luminance distribution of the image under consideration, and for the average distribution $\mu(x)$ we used the average luminance distribution of the entire set of 77 images. We again assumed a scale range of one, a dynamic range of one hundred, and one hundred items to be discriminated. Furthermore, we assumed equal weighting of discriminability and identifiability for overall importance $(\epsilon=0.5)$ and, in correspondence with what was found for the simplified case in the previous section, a relatively small degree of flexibility, $\lambda=0.25$. This particular choice for the degree of flexibility was also found to best describe experimental results in the colour domain (see chapter 4). Last, we checked for our set of 77 images whether the deviation $\Delta s(x, t)$ (equation 5.44) could indeed be adequately described by a Normal probability density function. This proved to be the case, and the spread $\hat{s}$ (equation 5.49) which we found for the set of images was approximately $0.128 \lambda$. Using equation 5.36, we arrived at a maximum number of identifiable items of approximately thirteen for the above choices of $\lambda=0.25$ and $P_{i}=25 \%$ (see also table 5.1).

Figure 5.4 shows judgments of visibility of detail (dotted lines) and sharpness (dashed lines) together with z-scored discriminability predictions (solid lines). For images 2 and 3 discriminability predictions closely correspond to sharpness judgments, for image 1 predictions are roughly midways the judgments of sharpness and visibility of detail, and for image 4 
predictions closely correspond to judgments of visibility of detail. The difference between sharpness and visibility of detail for values of $g$ larger than zero is probably due to two separate effects of the s-shaped transform. For small positive values of $g$ both sharpness and visibility of detail are increased due to increased contrast. However, for large positive values of $g$ visibility of detail is decreased due to the presence of increasingly larger areas in the manipulated images where all detail is lost due to clipping to either black or white. Sharpness, which is closely related to contrast, is not influenced much by the existence of such areas. It is not exactly clear to us why predictions correspond more closely to sharpness for some images and to visibility of detail for other images, and in the remainder of this discussion we have therefore chosen to consider the average of the judgments for sharpness and visibility of detail as an adequate correlate for discriminability.

Figure 5.5 shows naturalness judgments (dotted lines) and identifiability predictions (solid lines). Agreement between predictions and judgments is quite good for image 4, and somewhat less good for images 1, 2 and 3. The naturalness judgments for these three images show some asymmetry, with naturalness being judged best for small positive values of $g$. The position of the maximum of the naturalness judgments for these images nevertheless corresponds nicely with the position of the maximum of the identifiability predictions. The most important difference is the increased asymmetry of the identifiability predictions with respect to the naturalness judgments.

Figure 5.6 shows quality judgments (dotted lines) and predictions for total performance (solid lines). Here, agreement between predictions and judgments is quite good for all images. The only important difference is the over-estimation of the predictions for total performance with respect to quality judgments for image 2 for large positive values of $g$.

Figure 5.7 shows the average of the judgments for sharpness and visibility of detail (dotted lines), naturalness judgments (dashed lines) and quality judgments (solid lines). The figure shows that quality judgments lie approximately halfway naturalness and the average of sharpness and visibility of detail. This result strongly supports the central idea of this paper that quality is determined by both discriminability and identifiability.

To conclude, figure 5.8 shows predictions for discriminability (dotted lines), identifiability (dashed lines), and overall performance (solid lines). Compare the predictions in this figure with the experimental results in figure 5.7. Although smaller in size, the differences between discriminability 

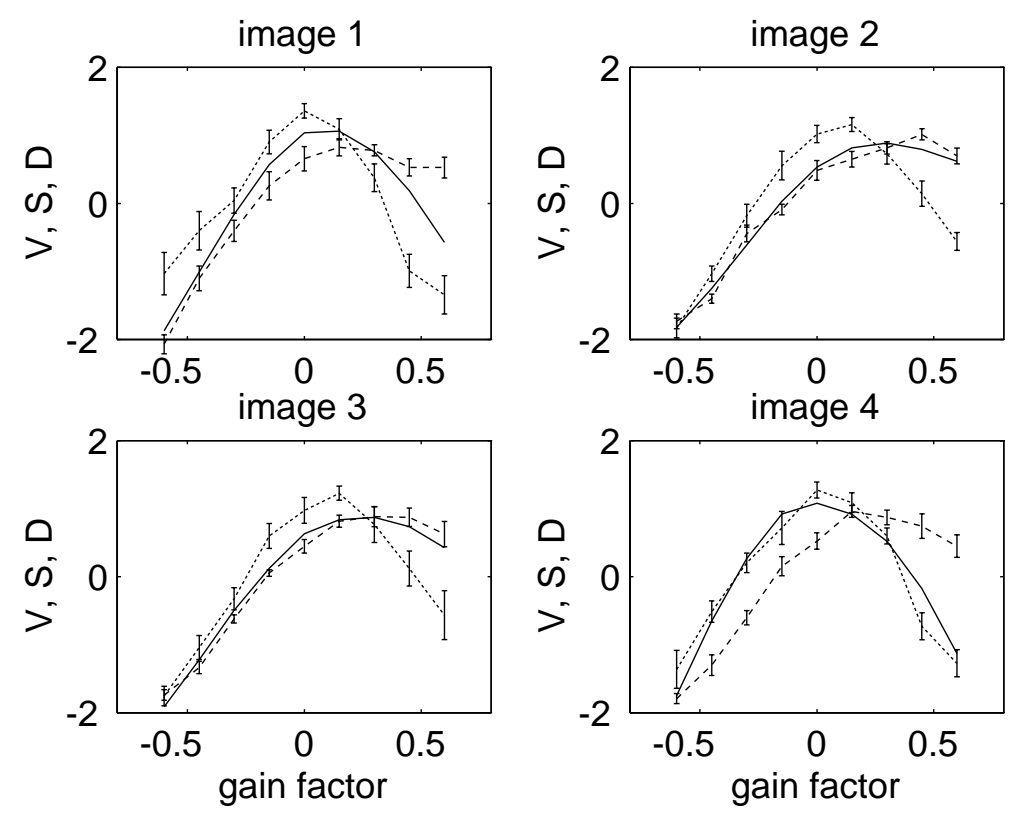

Figure 5.4: Experimentally obtained judgments for visibility of detail $V$ (dotted lines) and sharpness $S$ (dashed lines), versus the gain factor $g$ of the s-shaped transform. The error bars denote twice the standard error in the mean. The solid lines represent the predictions for discriminability $D$. Both judgments and predictions have been z-scored to facilitate comparison. 

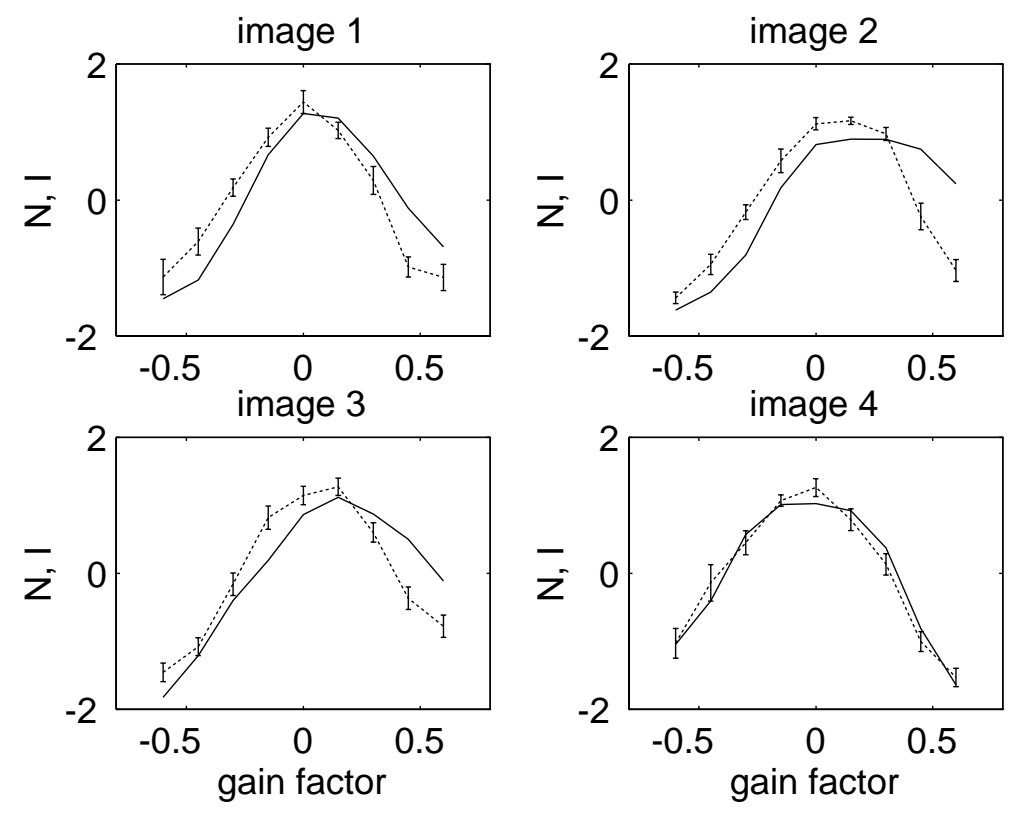

Figure 5.5: Experimentally obtained judgments for naturalness $N$ (dotted lines) and predictions for identifiability $I$ (solid lines), versus the gain factor $g$ of the s-shaped transform. The error bars denote twice the standard error in the mean. Judgments and predictions have been z-scored to facilitate comparison. 

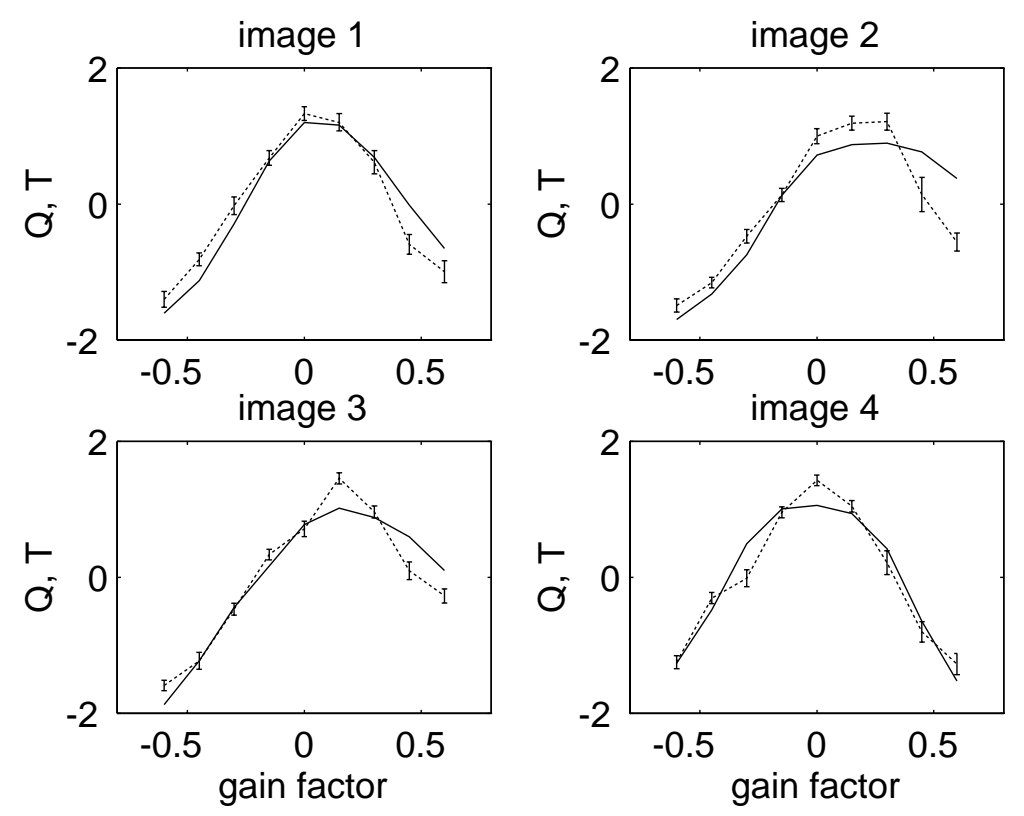

Figure 5.6: Experimentally obtained judgments for quality $Q$ (dotted lines) and predictions for total performance $T$ (solid lines), versus the gain factor $g$ of the s-shaped transform. The error bars denote twice the standard error in the mean. Judgments and predictions have been z-scored to facilitate comparison. 

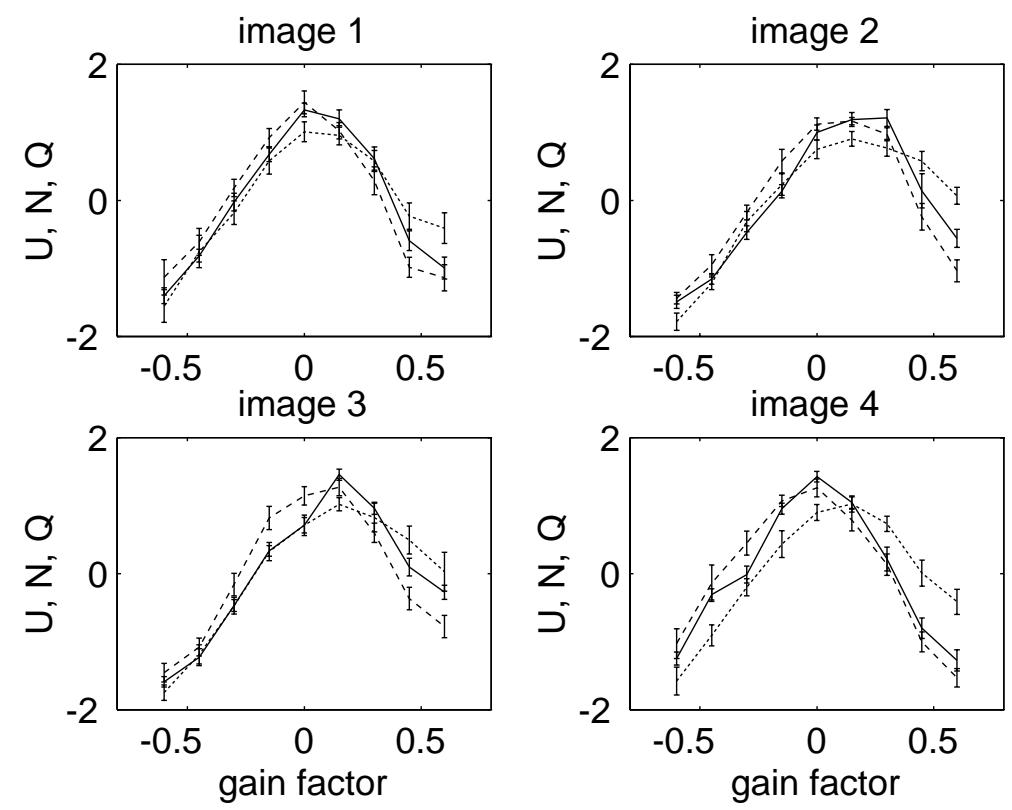

Figure 5.7: The average of the experimentally obtained judgments for visibility of detail and sharpness, $U$ (dotted lines), experimentally obtained judgments of naturalness $N$ (dashed lines), and experimentally obtained judgments of quality $Q$ (solid lines) versus the gain factor $g$ of the s-shaped transform. The error bars denote twice the standard error in the mean. All judgments have been z-scored to facilitate comparison. 


\subsection{Application: black-and-white images of natural scenes}
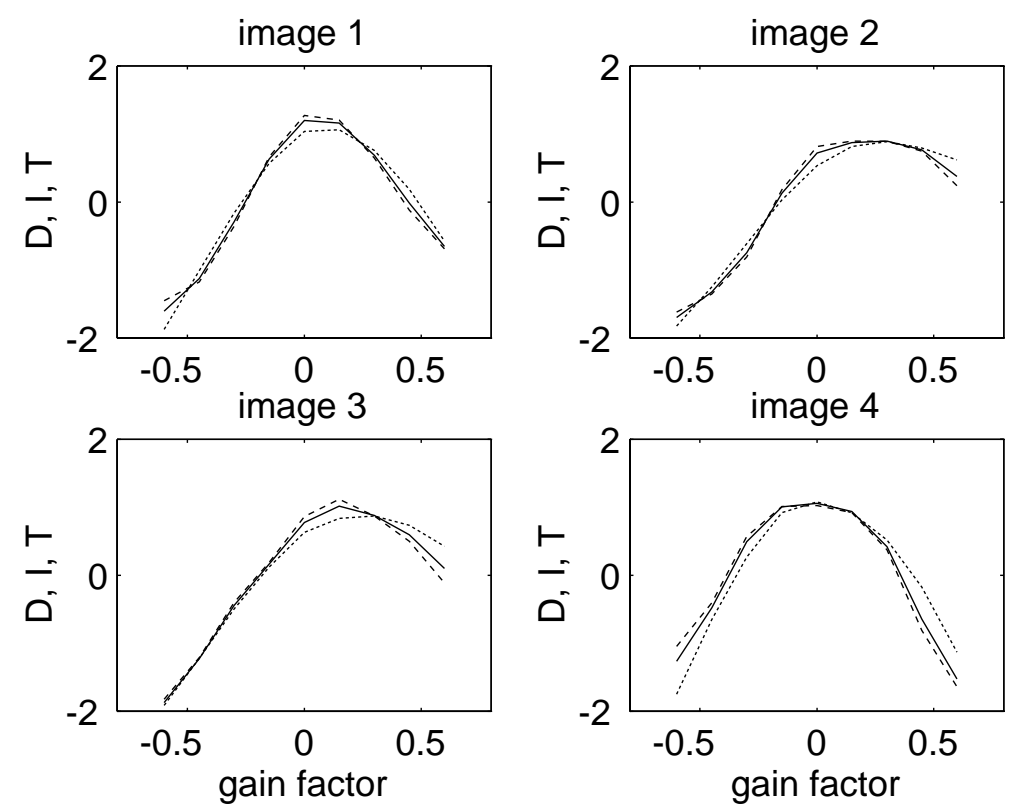

Figure 5.8: Predictions for discriminability $D$ (dotted lines), identifiability $I$ (dashed lines) and total performance $T$ (solid lines) versus the gain factor $g$ of the s-shaped transform.

and identifiability correspond nicely to the differences between the average of sharpness and visibility of detail and naturalness. Especially the small but systematic differences between naturalness and quality, with quality being higher than naturalness for large positive values of $g$ (all images) and quality being lower than naturalness for negative values of $g$ (most clearly in image 4), are correctly reflected in the predictions of identifiability and total performance. Therefore, although there are significant differences between experimental results and predictions, the validity of the concept we have developed here is supported by the correct prediction of several non-trivial characteristics of the obtained experimental results. 


\subsection{Conclusions}

We have presented a concept for image quality which is based on answering the questions: what are images, what are images used for, and what are the requirements which the use of images imposes on them. We have argued that images are the carriers of visual information, and that they are used as input to the perception stage of interaction. Furthermore, we have argued that the aim of perception is to measure and internally quantify attributes of items in the outside world in order to discriminate and identify these items. Therefore, the requirements imposed on an image are that, first, the items in the image should be discriminable and, second, the items in the image should be identifiable.

Since the internal quantification of outside world attributes is of such importance for a successful interaction, we have argued that the metrics used for this quantification should be of the type proposed in chapter 3 , that is, partially flexible and optimised with respect to discriminability and identifiability. We have derived expressions for such metrics, together with measures for discriminability, identifiability, and total performance. For the case of manipulated black-and-white reproductions of natural scenes, we have compared predictions made using these algorithms with experimentally obtained judgments of human subjects. Although there are significant differences between predictions and judgments, correspondence between predictions and judgments is quite good. Moreover, several important and non-trivial characteristics of the obtained judgments, such as the small but systematic difference between quality and naturalness judgments, are correctly predicted. 


\section{Chapter 6}

\section{Epilogue}

In this thesis a new concept for image quality has been presented. The concept is based on a top-down analysis of visuo-cognitive processing, in particular of the role of visuo-cognitive processing within the interaction process. Starting with a fundamental definition of quality in terms of the degree to which imposed requirements are satisfied, we have obtained a description of image quality which explicitly takes into account the fact that images are input to the vision stage of the interaction process. The result is a definition for image quality in terms of the adequacy of the image to serve as an input for the interaction process.

As stated in the conclusion of chapter 2, the concept we have presented here is basically formulated independently of modality, which opens the possibility to apply this concept to, for example, sound or speech quality, or to generalise it to the quality of multi-modal information presentation. An interesting possible extension mentioned in the conclusion of chapter 2 is a generalisation towards a concept for the quality of user-system interaction. The topic of this final chapter will be a short discussion of two (philosophical) issues related to the development of such a concept.

To start with the first issue, Newell \& Simon (1972) and Newell (1990) have argued convincingly that various aspects of human behaviour, such as reasoning, search, and problem solving, and therefore interaction in general, can be regarded as information-processing tasks. There is an important philosophical consequence to this position, namely that humans may be regarded as information-processing systems. Marr (1982) has argued that, in order to fully understand information-processing systems, these systems should be studied at the levels of semantics, algorithms, and implementation. Typical questions asked at these three levels are: what is 
the system doing, and why is it doing what it is doing (semantics), how is it doing what it is doing (algorithms), and what is it using to do what it is doing (implementation).

The second issue we wish to consider here is the structure of informationprocessing tasks, which seems to be strongly hierarchical. This is probably due to the fact that most information-processing tasks are performed by means of sequences of procedures, where each individual procedure often is an information-processing task in its own right. Hence, informationprocessing tasks can be subdivided into smaller tasks (subtasks) and are themselves part of larger tasks (supertasks). An important consequence of this hierarchical structure is that the goal of an information-processing task is in fact defined one level upwards in the hierarchy of informationprocessing tasks being performed.

The above two observations have a profound influence on the meaning of the concept quality within user-system interaction. Two consequences are particularly interesting here, and we will discuss them in order. The first is the fact that the concept of quality can be hierarchically defined across the three levels of semantics, algorithm, and implementation. The second is the fact that, at the semantic level, quality can only be meaningfully defined when the hierarchical structure of information processing is explicitly taken into account.

If we consider the semantic level, quality can be defined (as before in this thesis) in terms of the adequacy of the information-processing task for reaching the desired goal. Suitable criteria at the semantic level may for example be: has the desired goal been successfully accomplished (effectivity) and at what effort (efficiency). At the algorithmic level, quality refers to the adequacy of the algorithms which are performing the informationprocessing task, with criteria such as the ability to work on distorted input (robustness) or applicability to a wide range of different inputs (flexibility). Finally, at the level of implementation, quality refers to the adequacy of the hardware used to implement the information-processing algorithms. Typical criteria at this level are physical limits to the precision with which signals can be represented (noise), or representable signal strength (range).

The level of implementation is the level where quality is usually defined. Think, for example, of quality definitions for audio-visual equipment, where quality is defined in terms of limits to the precision and range with which signals can be represented. In this thesis we have successfully developed an alternative concept for image quality at the semantic level. We have made this choice for one very important reason: quality at the level 


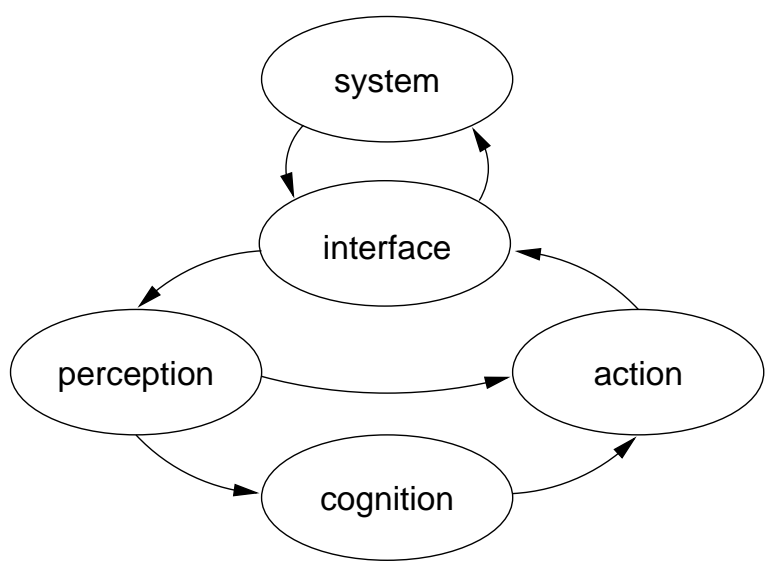

Figure 6.1: Anatomy of the user-system interaction cycle.

of implementation, or at the algorithmic level, is a necessary but insufficient condition for quality at the semantic level. In other words: although quality at the algorithmic and implementation level is important, and although quality at the semantic level depends upon quality at these two levels, it is quality at the semantic level which ultimately determines how well humans are able to use the image. This will not be different for a possible future concept for the quality of user-system interaction, where the userinterface takes the role the image has in the present concept.

As stated earlier, the nature of information-processing tasks, and hence of user-system interaction, is strongly hierarchical. We will start at what is perhaps the lowest distinguishable hierarchical level in user-system interaction: the level of the individual user-system interaction cycle. Furthermore, we will assume that the goal of user-system interaction at this lowest hierarchical level is to manipulate the system from its current state to some desired state, as specified one level upwards in the hierarchy. Figure 6.1 shows the anatomy of a single user-system interaction cycle.

The figure shows two flows of information between the user and the system, both of which are mediated by the user-interface. The information flow going from the system to the user is typically encoded visually, for example by means of images displayed on a screen, and is usually about the current status of the system. The information flow going in the opposite direction, from the user to the system, is usually encoded motori- 
cally, for example by means of mouse movements and mouse clicks, and often contains instructions from the user to the system. The user part of the interaction cycle in figure 6.1 is again divided into the stages perception, cognition, and action. Within this division, the aim of the perception stage is to "decode" the information flow from the system to the user. In the cognition stage, this decoded information is used to (re-)formulate the strategy for achieving the desired system state and deriving a procedure of instructions to the system. The aim of the action stage is then to "encode" these instructions into a format which can be interpreted by the interface and subsequently mediated to the system.

We have already observed that, at the semantic level, quality can only be meaningfully defined when the hierarchical structure of information processing tasks is taken into account. We are now at a point where we are able to explain why. To this end, consider that, at the level of implementation, quality may be defined in terms of the adequacy of the user-interface hardware as a means to mediate system status information to the user and user instructions to the system. Similarly, quality may be defined at the level of algorithms in terms of the adequacy of the formulated set of instructions to perform the task of manipulating the system from its present state to some new state, using the available user-interface. Finally, at the semantic level, quality may be defined in terms of the adequacy of the interaction strategy with respect to successfully, that is, effectively and efficiently, achieving the desired final system state. It is exactly for this that we need to know what the role of the interaction task is within its interaction supertask one level upwards in the hierarchy.

To conclude, the development of a concept for user-system interaction quality will require an in-depth analysis of the structure of the interaction process, as well as a careful analysis of the definitions for quality at the levels of semantics, algorithms, and implementation. A possible means to achieve this may be the definition of relatively welldefined, autonomous information-processing tasks at higher levels in the information-processing task hierarchy, such as the search task, similar to the discrimination and identification tasks we have defined earlier for visuo-cognitive processing. The knowledge thus obtained can be used to formulate requirements which should be imposed on user-interfaces in order to ensure a successful interaction, and subsequently to develop measures for quantifying the degree to which these requirements are satisfied. The availability of such measures will be an extremely important step towards the design of high-quality user-interfaces. 


\section{Bibliography}

Ballard, D. \& Brown, C. (1982). Computer vision, Prentice-Hall, Englewood Cliffs.

Barrow, H. \& Tenenbaum, J. (1986). Computational approaches to vision, in K. Boff, L. Kaufman \& J. Thomas (eds), Handbook of perception and human performance, Wiley-Interscience, Chichester, pp. 38.1-38.70.

Barten, P. (1990). Evaluation of subjective image quality with the squareroot integral method, Journal of the Optical Society of America A 7(10): 2024-2031.

Blommaert, F. (1995). Visual metrics, flexibility, and usefulness, Perception 24(supplement): 93.

Bruce, V. \& Green, P. (1985). Visual perception: physiology, psychology and ecology, Erlbaum, London.

Daly, S. (1993). The visible differences predictor: an algorithm for the assessment of image fidelity, in A. Watson (ed.), Digital images and human vision, MIT Press, Cambridge MA, pp. 179-206.

de Ridder, H. (1996). Naturalness and image quality: saturation and lightness variation in color images of natural scenes, Journal of Imaging Science and Technology 40(6): 487-493.

de Ridder, H., Fedorovskaya, E. \& Blommaert, F. (1993). Naturalness and image quality: chroma variations in colour images of natural scenes, IPO Annual Progress Report 28: 89-95.

Eimer, M. (1990). Representational content and computation in the human visual system, Psychological Research 52: 238-242.

Fechner, G. (1860). Elemente der Psychophysik, Breitkopf, Leipzig. 
Fedorovskaya, E., de Ridder, H. \& Blommaert, F. (1997). Chroma variations and perceived quality of color images of natural scenes, Color: Research and Application 22(2): 96-110.

Gibson, J. (1950). The perception of the visual world, Allen and Unwin, London.

Gibson, J. (1966). The senses considered as perceptual systems, Houghton Mifflin, London.

Gibson, J. (1979). The ecological approach to visual perception, Houghton Mifflin, London.

Hunt, R. (1992). Measuring colour, second edn, Ellis Horwood, Chichester.

Irving, J. \& Mullineux, N. (1959). Mathematics in physics and engineering, in H. Massey \& K. Bruecker (eds), Pure and applied physics, Vol. 6, Academic Press, New York.

Janssen, T. \& Blommaert, F. (1997). Image quality semantics, Journal of Imaging Science and Technology 41(5): 555-560.

Janssen, T. \& Blommaert, F. (1999a). A computational approach to image quality. Submitted for publication in Journal of the Optical Society of America A.

Janssen, T. \& Blommaert, F. (1999b). Predicting the usefulness and naturalness of colour reproductions. Accepted for publication in Journal of Imaging Science and Technology.

Janssen, T. \& Blommaert, F. (1999c). Visual metrics: discriminative power through flexibility. Submitted for publication in Perception.

Marr, D. (1982). Vision: a computational investigation into the human representation and processing of visual information, Freeman, San Francisco.

McCann, J. (1988). Calculated color sensations applied to image reproduction, Proceedings of the SPIE: image processing, analysis, measurement, and quality, Los Angeles, 13-15 January 1988, Vol. 901, SPIE, Bellingham, pp. 205-214.

Miller, G. (1956). The magical number seven, plus or minus two: some limits on our capacity for processing information, Psychological Review 63(2): 81-97. 
Newell, A. (1990). Unified theories of cognition, Harvard University Press, Harvard.

Newell, A. \& Simon, H. (1972). Human problem solving, Prentice-Hall, Englewood Cliffs.

Nijenhuis, M. \& Blommaert, F. (1997). Perceptual error measure for sampled and interpolated images, Journal of Imaging Science and Technology 41(3): 249-258.

Poggio, T. \& Koch, C. (1985). Ill-posed problems in early vision: from computational theory to analogue networks, Proceedings of the Royal Society of London B 226(1244): 303-323.

Poggio, T., Torre, V. \& Koch, C. (1990). Computational vision and regularization theory, in S. Ullman \& W. Richards (eds), Image understanding 1989, Ablex, Norwood, pp. 1-18.

Riesz, R. (1933). The relation between loudness and the minimum perceptible increment of intensity, Journal of the Acoustical Society of America 5: 211-216.

Roufs, J. (1993). Perceptual image quality: concept and measurement, Philips Journal of Research 47: 35-62.

Stevens, S. (1957). On the psychophysical law, Psychological Review 64: 153181.

Watt, R. (1989). Visual processing: computational, psychophysical and cognitive research, Erlbaum, Hove.

Watt, R. (1991). Understanding vision, Academic Press, London.

Weber, E. (1846). Der Tastsinn und das Gemeingefühl, Handwörterbuch der Physiologie 3: 481-588.

Whittle, P. (1994a). Contrast brightness and ordinary seeing, in A. Gilchrist (ed.), Lightness, brightness, and transparency, Erlbaum, Hillsdale, pp. 35-110.

Whittle, P. (1994b). The psychophysics of contrast brightness, in A. Gilchrist (ed.), Lightness, brightness, and transparency, Erlbaum, Hillsdale, pp. 111-157. 
Yendrikhovskij, S., Blommaert, F. \& de Ridder, H. (1998). Representation of memory prototype for an object colour. Accepted for publication in Color: Research and Application.

Yendrikhovskij, S., Blommaert, F. \& de Ridder, H. (1999). Colour reproduction and the naturalness constraint, Color: Research and Application 24(1): 52-67. 


\section{Sample stimuli}

The following pages show three sets of sample stimuli similar to those used in the experiments described in chapter 2. The first set (page 119) shows the effect of scaling CIE chroma, $C_{u v}^{*}$. The chroma scale factors used are: 0.50 (upper left), 0.71 (upper right), 1.00 (centre), 1.41 (lower left), and 2.00 (lower right). The second set (page 121) shows the effect of varying the colour temperature of the reference white, $T_{c}$. The colour temperatures used are: $4000 \mathrm{~K}$ (upper left), 5000K (upper right), 6500K (centre), 10000K (lower left), and 25000K (lower right). The third and last set (page 123) shows the effect of applying the s-shaped transformation on CIE lightness, $L^{*}$. The values of $\gamma$ used are: 0.25 (upper left), 0.50 (upper right), 1.00 (centre), 2.00 (lower left), and 4.00 (lower right). The corresponding values for the gain factor $g$ are $-0.60,-0.30,0.00,0.30$, and 0.60 , respectively. 

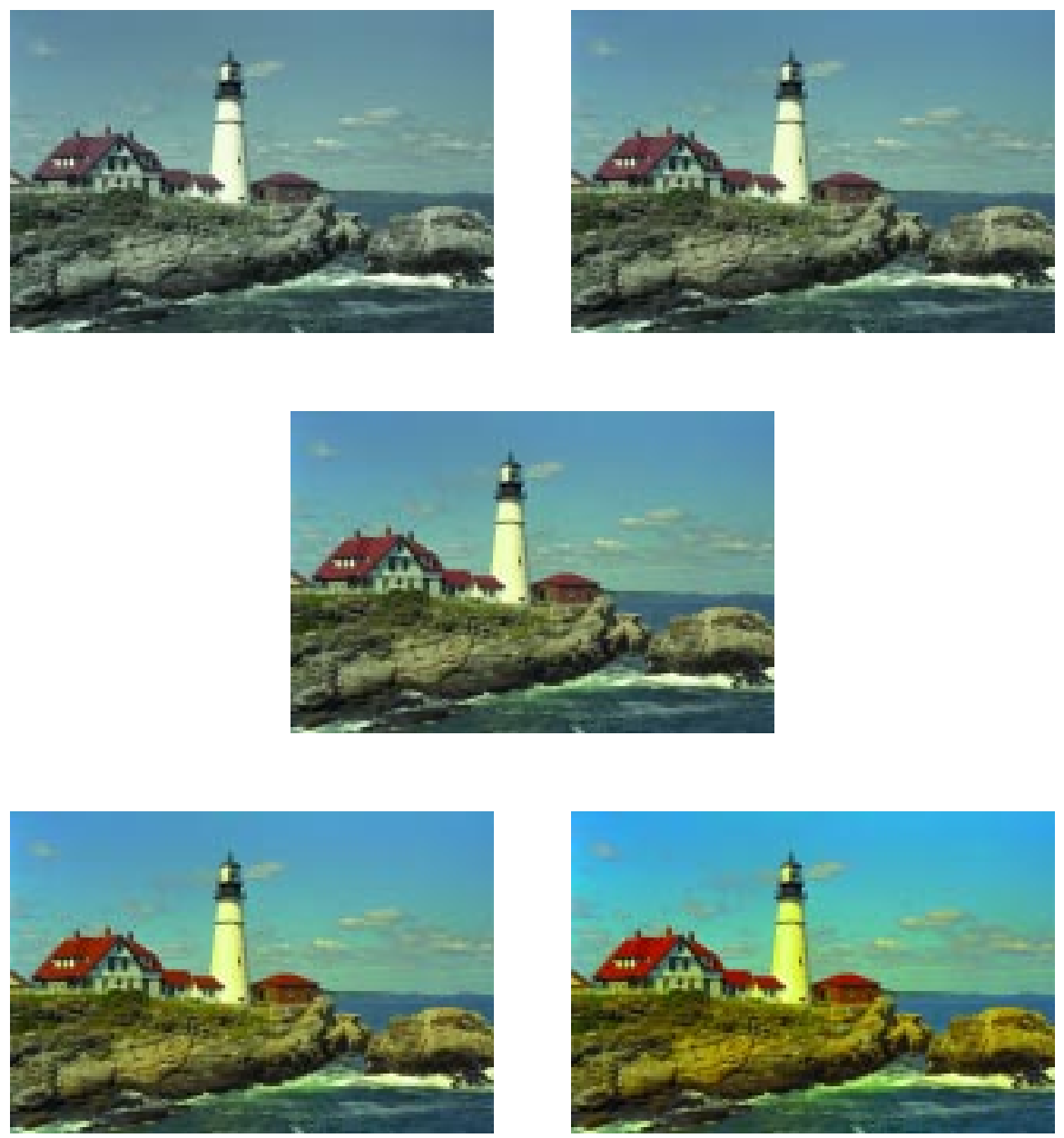

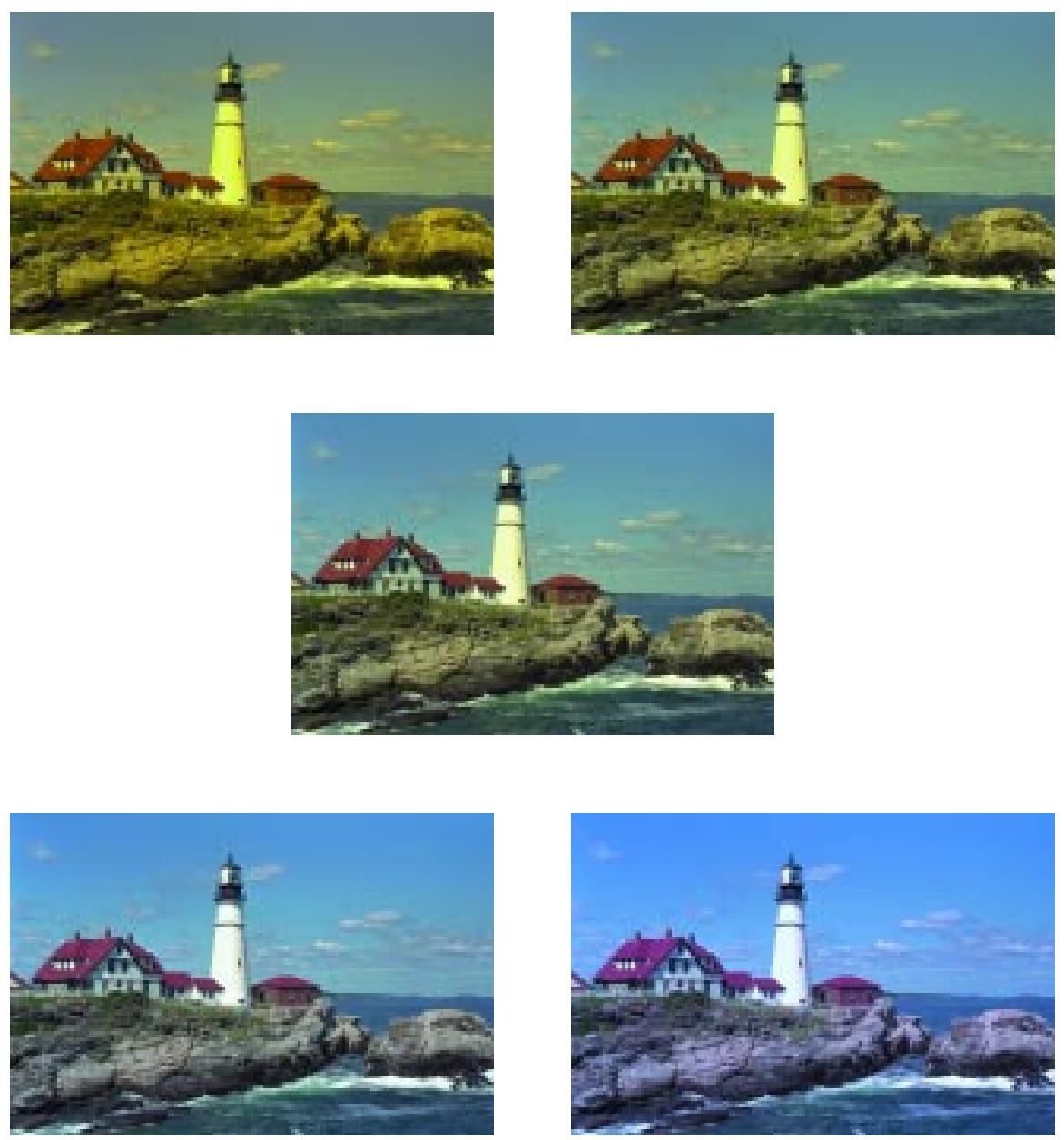

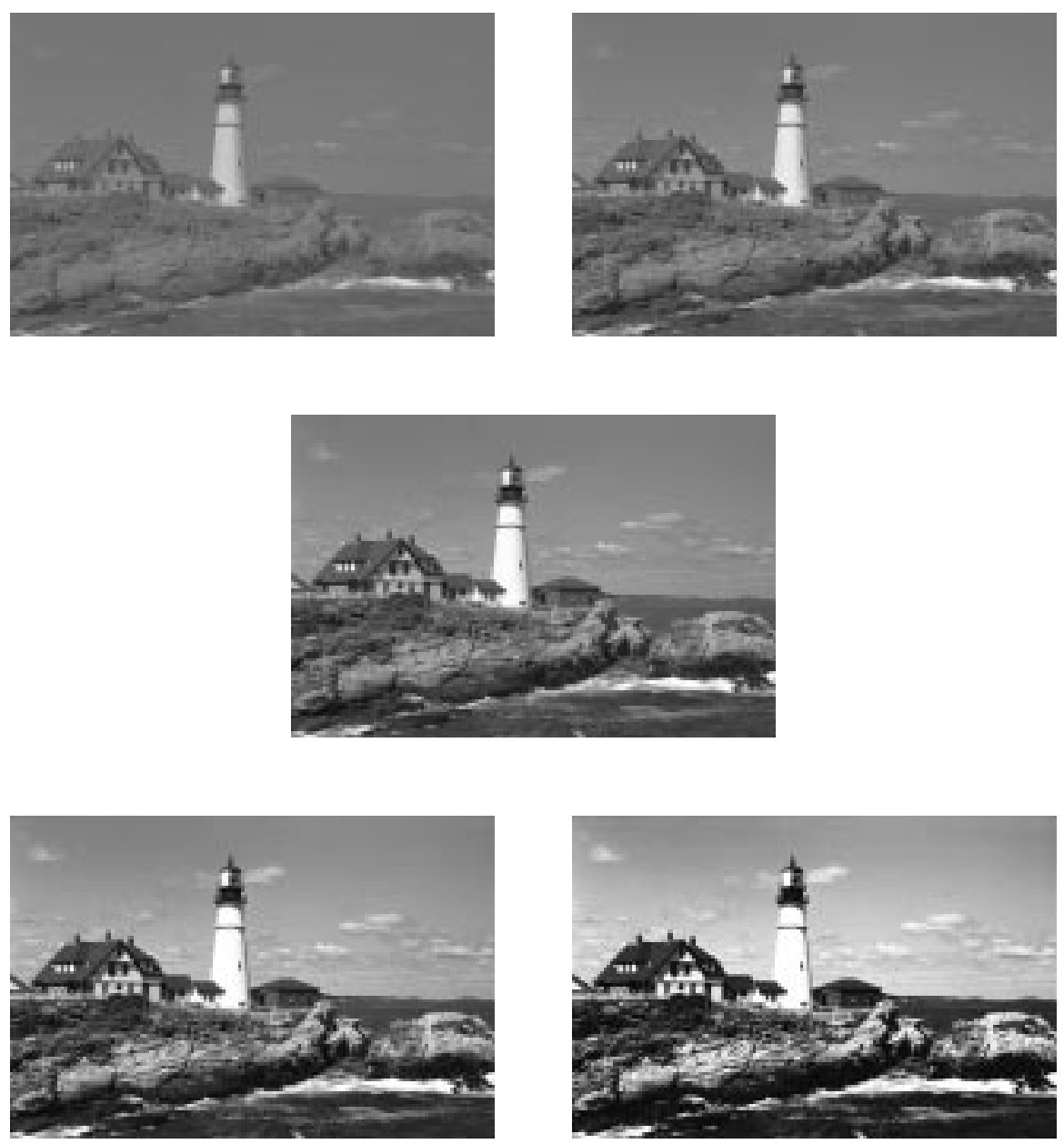


\section{Summary}

In this thesis an attempt is made to answer the fundamental question "What is image quality?". The approach we pursue to answer this question is based on the following four-point philosophy: (1) images are carriers of visual information; (2) visuo-cognitive processing is information processing; (3) visuo-cognitive processing is an essential stage in human interaction with the environment; and (4) image quality is the adequacy of the image as input to the vision stage of the interaction process.

The structure of this thesis is as follows. In chapter 2 we formulate an answer to the question "What is image quality?" based on the philosophy outlined above. In this chapter, we give a description of image quality in terms of two components, usefulness, that is, the precision of the internal representation of the image, and naturalness, that is, the degree of match between the internal representation of the image and representations stored in memory. The results of two series of experiments are used to demonstrate the validity of this concept.

In chapter 3 we focus on the internal quantification of outside world attributes. Using a rather technical view on visual processing, we regard vision primarily as a process in which attributes of items in the outside world are measured and internally quantified with the aim to discriminate and/or identify these items. We show that the scale function of metrics optimised with respect to these tasks should be (partially) flexible. Furthermore, we show that such metrics exhibit properties resembling visual phenomena such as adaptation, crispening, and constancy.

In chapter 4 we implement the image quality concept of chapter 2 using the partially flexible metrics of chapter 3 . In this chapter a measure for usefulness is developed, based on the overall discriminability of the items in the image. Furthermore, a measure for naturalness of the grass, skin, and sky areas of the image is developed, based on memory standards for grass, skin, and sky colour. These memory standards are themselves constructed 
from the grass, skin, and sky areas of a large set of images.

To conclude, chapter 5 returns to the concept for image quality introduced in chapter 2. Following a strict, top-down analysis the entire trajectory is completed from the semantics of image quality down to the development of algorithms for the prediction of usefulness, naturalness, and image quality. The result is a complete, thorough and explicit description of image quality according to the above four-point philosophy. 


\section{Samenvatting}

In dit proefschrift wordt een poging gedaan een antwoord te geven op de fundamentele vraag "Wat is beeldkwaliteit?". De aanpak die wij voor het beantwoorden van deze vraag volgen is gebaseerd op de volgende vierpunts filosofie: (1) beelden zijn dragers van visuele informatie; (2) visueelcognitieve processen zijn informatie-verwerkende processen; (3) visueelcognitieve processen vormen een essentieel onderdeel van menselijke interactie met de omgeving; en (4) beeldkwaliteit is de adequaatheid van het beeld als invoer voor het visueel-cognitieve onderdeel van het interactie proces.

De structuur van dit proefschrift is als volgt. In hoofdstuk 2 geven we een antwoord op de vraag "Wat is beeldkwaliteit?" dat gebaseerd is op de bovenstaande vier-punts filosofie. In dit hoofdstuk geven we een beschrijving van beeldkwaliteit uitgaande van twee componenten, usefulness (of bruikbaarheid), d.i. de precisie van de interne representatie van het beeld, en naturalness (of natuurlijkheid), d.i. de mate van overeenkomst tussen de interne representatie van het beeld en representaties die liggen opgeslagen in het geheugen. We gebruiken de resultaten van twee experimenten om de geldigheid van dit concept aan te tonen.

In hoofdstuk 3 concentreren we ons op de interne kwantificatie van attributen van de buitenwereld. We kiezen hier een tamelijk technische invalshoek op het visuele proces, en beschouwen het zien voornamelijk als een proces waarin attributen van items van de buitenwereld worden gemeten en intern gekwantificeerd met als doel het van elkaar onderscheiden en het identificeren van deze items. We tonen aan dat de schaalfuncties van metrieken die voor deze taken zijn geoptimaliseerd (gedeeltelijk) flexibel moeten zijn. Verder laten we zien dat zulke metrieken eigenschappen vertonen die overeenkomen met bekende visuele fenomenen zoals adaptatie, crispening, en constantie.

In hoofdstuk 4 implementeren we het concept voor beeldkwaliteit uit 
hoofdstuk 2 met behulp van de gedeeltelijk flexibele metrieken uit hoofdstuk 3. We ontwikkelen een maat voor bruikbaarheid die is gebaseerd op de onderscheidbaarheid van de items in een beeld. Bovendien ontwikkelen we een maat voor de natuurlijkheid van de gras-, huid-, en hemel-delen van een beeld die is gebaseerd op geheugen-standaards voor de kleur van gras, huid en hemel. De geheugen-standaards zelf zijn gebaseerd op de gras-, huid-, en hemel-delen van een grote set van beelden

In hoofdstuk 5 tenslotte keren we terug naar het concept voor beeldkwaliteit dat in hoofdstuk 2 werd geïntroduceerd. We kiezen in dit hoofdstuk voor een strikte top-down analyse en volgen zo het gehele traject van de semantiek van beeldkwaliteit tot aan de ontwikkeling van algoritmen voor het voorspellen van bruikbaarheid, natuurlijkheid en beeldkwaliteit. Het resultaat van deze aanpak is een complete, degelijke en expliciete beschrijving van beeldkwaliteit uitgaande van de bovenstaande vier-punts filosofie. 


\section{Biography}

Ruud Janssen was born on the 21st of February, 1970, in Laren. From 1982 to 1988 he attended Atheneum at "Laar en Berg" in Laren. Subsequently, from 1988 to 1994 he studied Electronic Engineering at the Eindhoven University of Technology (TUE), in Eindhoven, where he specialised in signal processing and medical electrical engineering. From 1994 to 1995 he completed alternative national service as a researcher in the former Vision Group at the Institute for Perception Research (IPO). At this same institute, he started the Ph.D. research described in this thesis in 1995. 
